
Le Groupe de travail sur la récupération des seringues usagées au Québec

a été mandaté par le directeur de la protection de la santé publique, Horacio Arruda, M.D.

\section{Composition du Groupe de travail}

Richard Cloutier, président

Agent de recherche et de planification sociosanitaire

Service de lutte contre les infections transmissibles sexuellement et par le sang

Direction de la protection de la santé publique

Ministère de la Santé et des Services sociaux

Lise Bertrand

Chef d'administration de programmes et responsable des soins infirmiers

CLSC-CHSLD du Marigot

Association des CLSC et des CHSLD du Québec

Marie-Josée Boulianne

Coordonnatrice de l'Unité des maladies infectieuses

Direction de santé publique de Laval

Table de concertation nationale en maladies infectieuses (TCNMI)

De juin 2003 à janvier 2004

Michèle Dupont

Médecin-conseil

Équipe risques biologiques en milieu de travail

Institut national de santé publique du Québec

Denis Laliberté

Médecin-conseil

Direction de santé publique de Québec

Table de concertation nationale en santé au travail (TCNSAT)

Johanne Milette

Infirmière-conseil

Direction de santé publique de la Mauricie et du Centre-du-Québec

Table de concertation nationale en maladies infectieuses (TCNMI)

De février à octobre 2004

Carole Morissette

Médecin-conseil

Direction de santé publique de Montréal

Table de concertation nationale en maladies infectieuses (TCNMI)

Manon Paul

Biologiste

Direction de santé publique des Laurentides

Table nationale de concertation en santé environnementale (TNCSE)

\section{Recherche et rédaction}

Hélène Gilbert

\section{Édition}

Direction des communications du ministère de la Santé et des Services sociaux

Pour obtenir un exemplaire de ce document, faites parvenir votre commande par télécopieur : (418) 644-4574

par courriel : communications@msss.gouv.qc.ca

ou par la poste: $\quad$ Ministère de la Santé et des Services sociaux

Direction des communications

1075, chemin Sainte-Foy, 16e étage

Québec (Québec) G1S 2M1

Ce document peut être consulté à la section Documentation, sous la rubrique Publications du site Web du ministère de la Santé et des Services sociaux dont l'adresse est : www.msss.gouv.qc.ca

Dépôt légal

Bibliothèque nationale du Québec, 2005

Bibliothèque nationale du Canada, 2005

ISBN 2-550-43418-8

Le genre masculin utilisé dans ce document désigne aussi bien les femmes que les hommes.

Toute reproduction totale ou partielle de ce document est autorisée, à condition que la source soit mentionnée.

(c) Gouvernement du Québec 


\section{Table des matières}

Liste des tableaux

Liste des sigles $\quad$ IX

Introduction 1

$1 \quad$ Le mandat et la démarche du groupe de travail 3

1.1 Le mandat 3

1.2 La démarche 4

2 Des raisons d'agir 7

2.1 Les piqûres accidentelles à l'extérieur du secteur de la santé et des services sociaux 7

2.2 Le coût des piqûres accidentelles 8

2.3 Les risques de transmission d'infections par une piqûre d'aiguille accidentelle $\quad 10$

2.4 Les conséquences d'une élimination non sécuritaire des seringues et des aiguilles $\begin{array}{ll}\text { pour certains services publics } & 12\end{array}$

$3 \quad$ L'utilisation de seringues et d'aiguilles 15

3.1 Les contextes d'utilisation d'instruments piquants et tranchants 15

3.2 Les quantités de seringues et d'aiguilles en cause 15

$4 \quad$ L'encadrement réglementaire 21

4.1 Le Règlement sur les déchets biomédicaux 21

4.2 Les Lignes directrices sur la récupération des seringues usagées 24

$5 \quad$ Les procédés utilisés pour le traitement et l'élimination des déchets $\begin{array}{ll}\text { biomédicaux } & 27\end{array}$

5.1 La décontamination par la vapeur 27

$\begin{array}{ll}5.2 \text { L'incinération } & 27\end{array}$

5.3 L'enfouissement sanitaire 28

$6 \quad$ Les pratiques de récupération et d'élimination des seringues et des aiguilles usagées chez les différents types d'utilisateurs $\quad 29$

6.1 Les centres hospitaliers 29

6.2 Les autres établissements publics de santé 30

$\begin{array}{lll}6.3 & \text { Les cliniques spécialisées } & 32\end{array}$

6.4 Les cliniques médicales privées 33

6.5 Les soins infirmiers privés 33

6.6 Les dentistes 33

6.7 Les acupuncteurs 34

6.8 Les pharmaciens 34

6.9 Les tatoueurs et les perceurs 36

$\begin{array}{lll}6.10 & \text { Les électrolystes } & 37\end{array}$

$\begin{array}{lll}6.11 & \text { Les thanatologues } & 37\end{array}$ 
6.12 Les vétérinaires 38

6.13 Les autres utilisateurs professionnels 38

6.14 Les utilisateurs de seringues et d'aiguilles pour les autosoins 38

6.15 Les usagers de drogues par injection 40

$7 \quad$ Divers modes de collaboration déjà expérimentés 43

7.1 Les centres d'accès aux seringues pour les UDI 43

7.2 Les services offerts par les CH munis d'autoclaves 44

7.3 La mobilisation de différents milieux pour la récupération des seringues souillées dans la région de la Mauricie et du Centre-du-Québec

7.4 Le comité de concertation pour la réduction des seringues à la traîne dans l'environnement à Montréal $\quad 45$

7.5 La récupération des seringues usagées de diabétiques en Abitibi-Témiscamingue 46

8 Des obstacles à surmonter 49

8.1 Un règlement qui ne s'applique pas aux individus 49

8.2 Des consignes contradictoires 49

8.3 Le refus de certains pharmaciens de récupérer les seringues et les aiguilles usagées $\quad 54$

8.4 Le refus de certains CLSC de récupérer les seringues et les aiguilles usagées 55

8.5 La non-disponibilité des autoclaves et le coût de la décontamination et de l'élimination des DBM 56

8.6 Le coût des contenants réglementaires 60

8.7 L'attitude répressive des policiers à l'endroit des UDI 62

8.8 Le manque de leadership et de coordination 63

9 Pour la mise en place d'un système intégré de récupération et d'élimination de toutes les seringues et de toutes les aiguilles usagées $\quad 65$

$9.1 \quad$ Le modèle général proposé $\quad 65$

9.2 Étendre l'application du Règlement sur les déchets biomédicaux à tous ceux qui font usage de seringues et d'aiguilles

9.3 Modifier le Règlement afin de faciliter la récupération des seringues et des aiguilles auprès des individus

9.4 Donner des consignes simples, les mêmes pour tous les utilisateurs de seringues et $\begin{array}{ll}\text { d'aiguilles à des fins domestiques } & 68\end{array}$

9.5 Généraliser l'utilisation des contenants réglementaires 70

9.6 Faciliter l'accès aux contenants réglementaires 70

9.7 Désigner des lieux où l'on serait tenu de récupérer les seringues et les aiguilles usagées

9.8 Faciliter l'accès aux équipements collectifs pour la décontamination et l'élimination des DBM

9.9 Offrir aux professionnels et aux travailleurs une formation et des mesures de $\begin{array}{ll}\text { prévention appropriées } & 81\end{array}$

9.10 Mener une campagne d'information adaptée aux différents publics concernés $\quad 82$

9.11 Concilier la protection de la santé publique et la protection de la sécurité publique 84 
9.12 Élaborer une politique nationale de récupération des seringues et des aiguilles usagées

9.13 Réunir les acteurs concernés dans chacune des régions $\quad 85$

$10 \quad$ Partager les responsabilités $\quad 87$

$\begin{array}{lll}10.1 & \text { Les individus } & 87\end{array}$

$\begin{array}{lll}10.2 & \text { Les CLSC } & 87\end{array}$

10.3 Les pharmaciens $\quad 88$

10.4 Les hôpitaux équipés d'autoclaves $\quad 88$

10.5 Les hôpitaux non pourvus d'autoclaves 89

10.6 Tous les hôpitaux $\quad 89$

10.7 Les cliniques spécialisées $\quad 89$

10.8 Les médecins 89

10.9 Les ordres professionnels, les corporations et les associations professionnelles 90

10.10 Les organismes communautaires 90

10.11 Les associations de malades 90

10.12 Les municipalités 90

10.13 Les services policiers 90

10.14 Les agences de développement de réseaux locaux de services de santé et de services sociaux 91

10.15 Le ministère de la Santé et des Services sociaux 91

10.16 Le ministère de l'Environnement 92

$\begin{array}{ll}\text { Conclusion } & 93\end{array}$

Annexes

I Des contributions précieuses 95

II Collecteur extérieur de seringues $\quad 107$

III Autoclave 109

IV Modifications proposées au Règlement sur les déchets biomédicaux 111

V Liste des recommandations $\quad 115$

VI Quelques autres questions soulevées au cours de nos consultations 119

$\begin{array}{ll}\text { Bibliographie } & 121\end{array}$ 



\section{Liste des tableaux}

\section{Tableau 1}

Nombre d'accidents de travail causés par des piqûres d'aiguilles ou d'aiguilles et seringues acceptés par la CSST et débours moyens par accident selon l'année de l'événement

Tableau 2

Nombre d'injections par jour et par année pour 80000 diabétiques

\section{Tableau 3}

Production annuelle totale de DBM provenant des autosoins requis pour les diabétiques qui s'injectent de l'insuline

\section{Tableau 4}

Les pharmacies qui distribuent des seringues aux UDI et celles qui les récupèrent en 2003-2004

\section{Tableau 5}

Avantages et inconvénients des diverses consignes données aux utilisateurs de seringues et d'aiguilles

\section{Tableau 6}

Présence d'autoclaves dans les réseaux locaux de services de santé et de services sociaux (juillet 2004)

\section{Tableau 7}

Avantages et inconvénients pour un $\mathrm{CH}$ de posséder son propre autoclave ou de confier ses DBM non anatomiques à l'entreprise privée

\section{Tableau 8}

Comparaison des prix des contenants de récupération pour DBM piquants et tranchants

\section{Tableau 9}

Coût des contenants pour DBM requis pour les diabétiques par année

\section{Tableau 10}

Endroits où pourraient être acheminés les DBM piquants et tranchants, selon le modèle proposé 



\section{Liste des sigles}

\begin{tabular}{|c|c|}
\hline ASSTSAS & $\begin{array}{l}\text { Association pour la santé et la sécurité du travail, secteur affaires } \\
\text { sociales }\end{array}$ \\
\hline CAS & Centre d'accès aux seringues \\
\hline $\mathbf{C H}$ & Centre hospitalier \\
\hline CHSLD & Centre d'hébergement et de soins de longue durée \\
\hline CHU & Centre hospitalier universitaire \\
\hline CHUM & Centre hospitalier de l'Université de Montréal \\
\hline CLSC & Centre local de services communautaires \\
\hline CPE & Centre de la petite enfance \\
\hline $\mathbf{C R}$ & Centre de réadaptation \\
\hline CS & Centre de santé \\
\hline CSSS & Centre de santé et de services sociaux \\
\hline CSST & Commission de la santé et de la sécurité du travail \\
\hline CUSM & Centre universitaire de santé McGill \\
\hline DBM & Déchets biomédicaux \\
\hline DSP & Direction de santé publique \\
\hline ITS & Infection transmissible sexuellement \\
\hline MENV & Ministère de l'Environnement \\
\hline MRC & Municipalité régionale de comté \\
\hline MSSS & Ministère de la Santé et des Services sociaux \\
\hline OC & Organisme communautaire \\
\hline OSBL & Organisme sans but lucratif \\
\hline PES & Programme d'échange de seringues \\
\hline RAMQ & Régie de l'assurance maladie du Québec \\
\hline RLSSSS & Réseau local de services de santé et de services sociaux \\
\hline UDI & Usager de drogues par injection \\
\hline UQAM & Université du Québec à Montréal \\
\hline VIH & Virus de l'immunodéficience humaine \\
\hline VHB & Virus de l'hépatite B \\
\hline VHC & Virus de l'hépatite C \\
\hline
\end{tabular}





\section{Introduction}

Des seringues sont trouvées près d'une garderie. Des résidants du secteur s'inquiètent et alertent la presse. Reportage alarmiste avec photos d'enfants à l'appui, interventions déchaînées d'un animateur de tribune téléphonique, interventions indignées de certains auditeurs : on alimente l'anxiété parmi la population. Invariablement, les toxicomanes sont montrés du doigt et il n'est pas rare que les programmes d'échange de seringues - une mesure mise en place par la santé publique pour réduire la transmission du VIH, de l'hépatite B et de l'hépatite C - soient mis en cause.

Le risque de contracter l'une de ces infections à la suite d'une piqûre accidentelle due à la présence d'une seringue dans l'environnement est considéré comme très faible. Toutefois, rien ne permet d'affirmer que ce risque est nul. Les conséquences psychologiques liées à la perception du risque peuvent aussi être importantes. Il faut donc prendre toutes les précautions nécessaires pour éviter ce type d'incident.

En y regardant de près, on constate que les UDI ne sont pas les seuls à ne pas toujours se débarrasser de leurs seringues de façon sécuritaire. D'autres utilisateurs de seringues et d'aiguilles, à des fins domestiques (p. ex. pour les autosoins) ou professionnelles, ont parfois des pratiques qui mettent en danger la santé et la sécurité de leur entourage et particulièrement celles de certains travailleurs. Outre les préposés à l'entretien des rues, des ruelles et des parcs, les concierges, les éboueurs ainsi que les employés des centres de tri figurent parmi les principales victimes des piqûres accidentelles qui surviennent à l'extérieur du secteur de la santé et des services sociaux.

Inconscience ou négligence de la part des utilisateurs de seringues et d'aiguilles ? Pas nécessairement. Au-delà des attitudes individuelles, les pratiques inadéquates ont souvent d'autres causes : lacunes dans la réglementation, absence d'équipement ou de service accessible, confusion dans les consignes données, etc.

Pour résoudre ce problème - plus complexe qu'il n'y paraît à première vue - le Groupe de travail sur la récupération des seringues usagées au Québec a d'abord voulu tracer le portrait le plus fidèle possible de la situation. Il a voulu connaître notamment les quantités de seringues et d'aiguilles en cause, les pratiques de récupération des différents utilisateurs de ces instruments et les divers modes de collaboration déjà expérimentés. À cette fin, il a consulté de nombreux acteurs concernés. L'information obtenue a permis de déceler les principaux obstacles à l'élimination sécuritaire des seringues et des aiguilles usagées. Le groupe de travail a ensuite examiné diverses façons de surmonter ces obstacles avant de formuler ses recommandations. Il en conclut que chacun des acteurs doit être mis à contribution: l'élimination sécuritaire des seringues et des aiguilles usagées, c'est une responsabilité à partager. 



\section{Le mandat et la démarche du groupe de travail}

L'intervention visant la récupération des seringues usagées est inscrite au Programme national de santé publique 2003-2012 parmi les actions susceptibles de prévenir les infections transmissibles par le sang telles que le VIH, l'hépatite B et l'hépatite C.

Le Groupe de travail sur la récupération des seringues usagées au Québec a été formé par le directeur de la protection de la santé publique du ministère de la Santé et des Services sociaux (MSSS) au printemps 2003, avec la collaboration de la Table de concertation nationale en maladies infectieuses (TCNMI), de la Table de concertation nationale en santé au travail (TCNSAT) et de la Table nationale de concertation en santé environnementale (TNCSE).

Constitué de professionnels provenant de directions régionales de santé publique (secteurs maladies infectieuses, santé en milieu de travail et santé environnementale), de l'Institut national de santé publique du Québec, de l'Association des CLSC et des CHSLD du Québec et du Service de lutte contre les maladies transmissibles sexuellement et par le sang, le groupe de travail s'est réuni pour la première fois le 16 juin 2003. Il a tenu au total dix rencontres qui ont servi essentiellement à orienter la démarche et à valider les travaux.

\subsection{Le mandat}

Le mandat confié au groupe de travail était formulé ainsi :

Le groupe de travail devra recommander à la Direction de la protection de la santé publique du MSSS les actions qui doivent être mises en place pour réduire le nombre de seringues dans l'environnement et faciliter la récupération du matériel d'injection usagé.

Plus précisément, il devra :

a) analyser les lois et règlements en vigueur relativement à la récupération de seringues et relever leurs forces et leurs lacunes ;

b) déterminer des modalités favorisant l'établissement d'une politique de récupération des seringues usagées par les CLSC, les $\mathrm{CH}$, les pharmacies et les cliniques médicales privées ;

c) élaborer une politique de récupération des seringues dans les lieux publics et dans les lieux privés accessibles au public ;

d) définir une politique de gestion des seringues à usage domestique à des fins thérapeutiques ;

e) déterminer les mesures à mettre en œuvre pour la protection des travailleurs pouvant être exposés à des seringues dans l'environnement ;

f) estimer les ressources financières nécessaires à l'application de ces recommandations.

Au moment d'élaborer notre plan de travail, nous avons cru bon d'élargir la problématique à d'autres instruments susceptibles de causer des piqûres accidentelles et, éventuellement, de transmettre des infections: aiguilles utilisées en acupuncture, aiguilles employées pour le tatouage et le perçage, filaments en usage chez les électrolystes, aiguilles pour stylo injecteur et lancettes servant aux diabétiques. À moins que le contexte ne s'y oppose, l'expression seringues et aiguilles, employée dans ce document pour éviter d'alourdir le texte, désignera l'ensemble de ces instruments. 
Dès le début de nos travaux, nous avons constaté que les différents éléments du mandat étaient étroitement liés, que la résolution des problèmes exigeait la mise en place d'un système intégré, c'est-à-dire d'une organisation permanente selon laquelle les opérations reliées à chacune des étapes de gestion des seringues et des aiguilles seraient cohérentes. Les différentes mesures proposées viseront donc à ce que toutes les seringues et toutes les aiguilles utilisées au Québec soient récupérées et éliminées de façon à protéger à la fois la population en général, les travailleurs et l'environnement.

\subsection{La démarche}

Dans le but de tracer le portrait le plus fidèle possible de la réalité, nous avons d'abord réalisé des enquêtes au moyen de trois questionnaires écrits adressés respectivement aux directions de santé publique (DSP), aux CLSC et CHSLD ${ }^{1}$, puis aux centres hospitaliers $(\mathrm{CH})^{2}$.

Par ces enquêtes, nous voulions connaître la perception de l'importance du problème de la récupération et de l'élimination des seringues usagées dans les différents milieux, les procédés utilisés et leur coût, les consignes données au personnel et aux usagers, les collaborations établies, les actions réalisées, les difficultés éprouvées, les responsabilités que chacun se reconnaît, les besoins à combler, etc.

Les taux de réponses à ces enquêtes - dont les résultats ont été transmis aux directions de santé publique de chacune des régions - ont été les suivants :

- DSP : 18 sur 18 (100\%), 48 participants ;

- CLSC et CHSLD : 128 établissements sur 193 (66,3\%), 424 participants ;

- $\mathrm{CH}$ : 85 établissements sur 110 (77\%), 166 participants.

Nous avons aussi consulté divers acteurs dans ce dossier, aux niveaux local et national, généralement lors de rencontres individuelles ou collectives à leur lieu de travail. Quelques personnes ont été interviewées par téléphone ; quelques autres ont répondu à un bref questionnaire acheminé par l'entremise de leur association. Nous avons ainsi pu connaître les expériences vécues, les difficultés éprouvées ou les solutions envisagées par:

- ceux qui utilisent des seringues et des aiguilles à des fins professionnelles : cliniques médicales, soins infirmiers privés, cliniques spécialisées, dentistes, acupuncteurs, thanatologues, vétérinaires, tatoueurs, perceurs, électrolystes, centre de recherche, Héma-Québec, Urgences-santé ;

- ceux qui oeuvrent auprès des utilisateurs de seringues et d'aiguilles à des fins domestiques : associations de malades, organismes communautaires, centres d'accès aux seringues ;

- ceux qui vendent des seringues, des aiguilles et des contenants de récupération: fabricants, distributeurs, pharmaciens ;

- ceux qui sont aux prises avec les problèmes résultant d'une mauvaise gestion des seringues et des aiguilles usagées : services de garde à l'enfance, milieux de l'éducation, Postes Canada, municipalités ;

1. Pour cette enquête, nous avons bénéficié de l'appui de l'Association des CLSC et des CHSLD du Québec.

2. Pour cette enquête, nous avons bénéficié de l'appui de l'Association des hôpitaux du Québec. 
- ceux qui procèdent au traitement, au transport et à l'élimination des déchets biomédicaux : hôpitaux munis d'autoclaves, Services médicaux Med-Tech, site d'enfouissement ;

- autres : Association des hôpitaux du Québec, agences de développement de réseaux locaux de services de santé et de services sociaux, Info-Santé CLSC, Association des directeurs de police du Québec.

D'autres encore, soit les hôpitaux pour enfants, la Commission de la santé et de la sécurité du travail et le ministère de l'Environnement, nous ont fourni de précieux renseignements.

Au total, plus de 800 personnes ont été directement associées à la démarche du Groupe de travail sur la récupération des seringues usagées au Québec. La liste des DSP et des établissements qui ont répondu aux questionnaires de même que celle des personnes consultées lors de rencontres ou d'entrevues téléphoniques se trouvent à l'annexe I. 



\section{Des raisons d'agir}

La majorité des accidents de travail impliquant des seringues et des aiguilles acceptés ${ }^{3}$ par la Commission de la santé et de la sécurité du travail (CSST) surviennent dans le secteur de la santé et des services sociaux. Même si ces piqûres accidentelles représentent un sérieux problème, le groupe de travail ne s'est pas penché sur la question puisque d'autres instances, telle l'Association pour la santé et la sécurité du travail, secteur affaires sociales (ASSTSAS), assument cette mission.

\subsection{Les piqûres accidentelles à l'extérieur du secteur de la santé et des services sociaux}

Les travailleurs et travailleuses de la santé sont loin d'être les seules victimes des piqûres accidentelles. Les données qui suivent illustrent l'importance d'agir dès maintenant, en matière de récupération et d'élimination des seringues et des aiguilles, afin de protéger la population en général, notamment les enfants, ainsi que les travailleurs et travailleuses.

- De 1998 à 2002, sur les 1426 accidents de travail causés par des aiguilles ou des aiguilles et seringues acceptés par la CSST, $503(35 \%)$ touchaient des personnes travaillant à l'extérieur du réseau de la santé et des services sociaux ${ }^{4}$.

- De 1999 à 2002, le centre de référence et de prophylaxie postexposition de l'hôpital Saint-Luc, à Montréal, a enregistré 1160 expositions percutanées et mucocutanées ${ }^{5}$. De ce nombre, 609 (52\%) sont survenues à l'extérieur des milieux de soins. Dans ces cas, les lieux d'exposition mentionnés étaient les suivants : école, garderie, parc, rue, autobus, métro, restaurant, domicile, pharmacie, voiture de police, poste de police, palais de justice ou centre de détention.

- De 1995 à 2003, l'hôpital Sainte-Justine a traité 148 enfants à la suite de "piqûres d'aiguille accidentelles dans la communauté ». Parmi les cas où le lieu de l'accident était connu, 73 piqûres étaient survenues à des endroits tels que rue ou ruelle, parc, restaurant, aréna, camping ou stationnement ; 15 s'étaient produites à domicile, et 45 , à d'autres endroits que l'on présume sécuritaires pour les enfants: garderie, école, autobus scolaire, cabinet de médecin, CLSC ou hôpital ${ }^{6}$.

- Entre le $1^{\text {er }}$ janvier 1988 et le 30 octobre 2003, l'urgence de l'hôpital de Montréal pour enfants a répertorié 92 cas de "piqûres d'aiguille accidentelles dans la communauté ». Les lieux (connus) où les accidents s'étaient produits étaient les suivants : rue ou ruelle, parc, domicile, restaurant, école, garderie et stationnement ${ }^{7}$.

Heureusement, aucun cas de séroconversion n'a été rapporté au Québec à la suite de piqûres accidentelles survenues à l'extérieur du secteur de la santé et des services sociaux. Mais combien d'infections ont été évitées grâce aux mesures de prophylaxie postexposition appliquées à la suite

3. Ce terme signifie que l'accident a entrainé des débours de la part de la CSST.

4. Information obtenue à partir de données fournies par la CSST.

5. La très vaste majorité de ces expositions étaient percutanées et l'objet en cause était une aiguille. Source : données de surveillance du centre de référence et de prophylaxie postexposition de l'hôpital Saint-Luc.

6. Information transmise par Denis Blais, service des maladies infectieuses, hôpital Sainte-Justine, Montréal.

7. Jesse PAPENBURG, Caroline QUACH, Mohammed AL-HOSNI et Dorothy MOORE, Pediatric community-acquired needlestick injuries: description and risk of seroconversion, hôpital de Montréal pour enfants, CUSM, Montréal, 2004. 
de ces accidents ? Difficile de le savoir. Nous savons cependant que ces accidents ont des coûts humains (angoisse de la personne victime de l'accident et de son entourage, nécessité de protection sexuelle, effets secondaires des médicaments, etc.) et des coûts économiques qui pourraient être épargnés par une gestion sécuritaire des seringues et des aiguilles usagées.

\subsection{Le coût des piqûres accidentelles}

$\mathrm{Au}$ Québec, on recommande aux personnes victimes de piqûres accidentelles de consulter un professionnel de la santé le plus tôt possible, idéalement dans un délai de deux heures après l'accident. Chaque cas doit être évalué afin de déterminer les mesures à prendre afin d'éviter que la personne contracte une infection.

Le coût de la prise en charge d'une exposition au sang varie considérablement selon que la source (personne piquée à l'origine avec cette aiguille) est connue ou non et, lorsque la source est connue, selon qu'elle est considérée comme étant à risque d'être porteuse du VIH, du VHB ou du VHC ou encore qu'elle est déjà atteinte de l'une de ces infections.

Dans tous les cas déclarés, du temps sera passé au moins par la personne blessée (et par ses parents, s'il s'agit d'un enfant) pour se rendre à l'hôpital ou au CLSC, ainsi que par le personnel médical, pour évaluer la situation. L'accident peut cependant engendrer des coûts beaucoup plus élevés pour la personne blessée, pour son employeur, pour le réseau de la santé et, finalement, pour la société dans son ensemble.

Par exemple, dans le cas d'un accident de travail touchant un travailleur affecté à la collecte des ordures ménagères, les coûts peuvent comprendre les éléments suivants ${ }^{8}$ :

Coûts initiaux

- déclaration / traitement (temps du travailleur, du responsable de la déclaration CSST, du personnel de l'urgence, des professionnels consultés, etc.) ;

- prophylaxie postexposition (vaccins, antirétroviraux, etc.) ;

- épreuves de laboratoire initiales - travailleur;

- épreuves de laboratoire - personne source, si connue.

Coûts du suivi

- épreuves de laboratoire - travailleur ;

- évaluation / consultations (temps du travailleur impliqué), temps pour prélèvement et counselling, consultations de professionnels (médecin, spécialiste en maladies infectieuses, pharmacien, psychologue, travailleur social, etc.).

Coûts de la lésion, si absence

- remplacement du revenu de la personne absente ;

- salaire du suppléant.

8. Cette liste est inspirée d'un article signé par Françoise BOUCHARD, intitulé « Une exposition au sang pour un travailleur: impacts économiques », paru dans le magazine de l'ASSTSAS, Objectif prévention, vol. 25, $\mathrm{n}^{\circ} 2,2002$, p. 9. 
Comme l'indique le tableau 1, au cours de la période 1998 à 2002, la CSST a déboursé un total de 2021358 \$ pour les 1426 accidents de travail causés par des piqûres d'aiguilles ou d'aiguilles et seringues. Parmi ces accidents, 503 sont survenus à l'extérieur du secteur de la santé et des services sociaux. Ils ont coûté à la CSST un total de 848835 \$, soit une moyenne de $1687,54 \$$ chacun. Pour les accidents qui se sont produits dans le secteur de la santé et des services sociaux, le total des débours de la CSST s'est élevé à 1172523 \$, soit en moyenne 1270,34 \$ par accident. La différence peut sans doute s'expliquer par le fait que, lorsqu'un accident survient dans le milieu de la santé, certains services sont disponibles sur place, ce qui évite des frais liés au déplacement de la personne concernée.

On constate par ailleurs une augmentation constante du nombre d'accidents «acceptés », ce nombre ayant plus que doublé en cinq ans. Explication probable : les gens, étant maintenant plus sensibilisés aux risques liés aux piqûres d'aiguilles accidentelles, déclarent des accidents qu'auparavant on aurait passés sous silence. Il existe sans doute encore une sous-déclaration des accidents, mais nous n'en connaissons pas l'ampleur.

\section{Tableau 1}

Nombre d'accidents de travail causés par des piqûres d'aiguilles ou d'aiguilles et seringues acceptés* par la CSST et débours moyens par accident selon l'année de l'événement

\begin{tabular}{lcccccccccc}
\hline $\begin{array}{c}\text { Année de } \\
\text { l'événement }\end{array}$ & \multicolumn{2}{c}{$\begin{array}{c}\text { Secteur de la santé et des } \\
\text { services sociaux }\end{array}$} & \multicolumn{3}{c}{ Autres secteurs } & \multicolumn{3}{c}{ Total } \\
\hline & $\mathbf{N}^{\text {bre }}$ & $\begin{array}{c}\text { Débours } \\
\text { totaux \$ }\end{array}$ & $\begin{array}{c}\text { Débours } \\
\text { moyens \$ }\end{array}$ & $\mathbf{N}^{\text {bre }}$ & $\begin{array}{c}\text { Débours } \\
\text { totaux \$ }\end{array}$ & $\begin{array}{c}\text { Débours } \\
\text { moyens \$ }\end{array}$ & $\mathbf{N}^{\text {bre }}$ & $\begin{array}{c}\text { Débours } \\
\text { totaux \$ }\end{array}$ & $\begin{array}{c}\text { Débours } \\
\text { moyens \$ }\end{array}$ \\
\hline 1998 & 120 & 117365 & 978,04 & 69 & 57806 & 837,77 & 189 & 175171 & 926,83 \\
\hline 1999 & 118 & 107709 & 912,79 & 88 & 167394 & 1902,20 & 206 & 275103 & 1335,45 \\
\hline 2000 & 157 & 356375 & 2269,90 & 98 & 206972 & 2111,96 & 255 & 563347 & 2209,20 \\
\hline 2001 & 254 & 310031 & 1220,60 & 117 & 215840 & 1845,42 & 371 & 525871 & 1417,44 \\
\hline 2002 & 274 & 281043 & 1025,70 & 131 & 200822 & 1533,63 & 405 & 481865 & 1189,79 \\
\hline TOTAL & 923 & 1172523 & 1270,34 & 503 & 848835 & 1687,54 & 1426 & 2021358 & 1417,50 \\
\hline
\end{tabular}

Source : Commission de la santé et de la sécurité du travail.

* Le terme accepté signifie qu'il y a eu débours de la part de la CSST.

Le coût d'une seule piqûre accidentelle sans séroconversion peut se situer entre quelques centaines et plusieurs milliers de dollars, compte tenu de l'absence plus ou moins prolongée que la situation du travailleur (effets secondaires des médicaments, état psychologique) peut exiger. Et que dire des coûts qui seraient engendrés par une séroconversion, voire par le décès du travailleur à la suite de cette piqûre accidentelle? 


\subsection{Les risques de transmission d'infections par une piqûre d'aiguille accidentelle}

Nous l'avons vu, les piqûres accidentelles causées par des seringues et des aiguilles usagées suscitent beaucoup de craintes. Mais, quel est le risque réel de contracter le VIH, le VHB ou le VHC par ce type de blessure ? Cela va sans dire que la seringue ou l'aiguille à l'origine de l'accident doit avoir été en contact avec le sang d'une personne infectée par l'un de ces virus. Toutefois, cette condition est loin d'être suffisante pour que la piqûre implique un risque réel de transmission de l'infection. Plusieurs autres facteurs sont à considérer, dont le degré de viabilité de chacun de ces virus à l'extérieur du corps humain dans diverses conditions (p. ex. selon la température).

Une étude réalisée sous la direction du $\mathrm{D}^{\mathrm{r}}$ Pierre Robillard ${ }^{9}$ en 1997 a permis de détecter des traces du VHB et du VHC (antigène HBsAg ou ADN du VHB et ARN du VHC) dans des seringues laissées durant plusieurs jours (jusqu'à 29 jours) dans l'environnement ou en laboratoire. Une étude menée en 1994 par l'Institut Pasteur a montré que $12 \%$ des seringues en provenance de lieux publics et $10 \%$ des seringues ramassées sur les plages contenaient des anticorps antiVIH contre $54 \%$ des seringues en provenance de programmes d'échange ${ }^{10}$.

La viabilité d'un virus ne correspond pas nécessairement à la période durant laquelle il est capable de transmettre une infection. D'autres études citées par le $\mathrm{D}^{\mathrm{r}}$ Robillard indiquent que, dans des conditions expérimentales, des seringues contaminées avec du sang infecté par le VIH, le VHB ou le VHC peuvent demeurer infectieuses durant 8 mois dans le cas du VHB et du VHC (p. 7) et durant au moins un mois dans le cas du VIH (p. 8). Cela ne signifie pas qu'il en soit de même pour les seringues contaminées laissées à des endroits inappropriés (parcs, rues, ordures ménagères, etc.).

Divers facteurs influencent le niveau de risque de transmission d'une infection : présence de sang sur l'instrument, prévalence de l'infection, charge virale, temps écoulé depuis l'utilisation de la seringue ou de l'aiguille, profondeur de la blessure, niveau d'immunité de la personne blessée, etc. Plusieurs de ces facteurs sont plus facilement vérifiables lorsqu'un accident se produit à l'occasion de l'administration de soins de santé (injection, prélèvement sanguin, etc.) puisque, normalement, la personne source (celle qui a été piquée à l'origine) est connue.

En l'absence de prophylaxie postexposition, les risques de contracter une infection à la suite d'une piqûre accidentelle dans les milieux de soins de santé sont les suivants :

- hépatite $\mathrm{B}$ : de 2 à $40 \%$, selon le niveau d'infection de la personne source ;

- hépatite $\mathrm{C}$ : environ $3 \%$;

- $\mathrm{VIH}: 0,3 \%(3 \text { sur } 1000)^{11}$.

9. Pierre ROBILLARD, Évaluation de l'infectiosité des seringues à la traîne dans l'environnement: rapport d'étude pilote, Montréal, septembre 1998, $34 \mathrm{p}$.

10. F. SQUINAZZI, S. CHAMARET, Y. A. COURTOIS et L. MONTAGNIER, « La présence d'anticorps anti-VIH dans des seringues usagées abandonnées sur la voie publique ou recueillies lors de programmes d'échange pour toxicomanes : Résumé », cité dans MINISTĖRE DE L'EMPLOI ET DE LA SOLIDARITÉ, SECRÉTARIAT D'ÉTAT À LA SANTÉ ET À L'ACTION SOCIALE. Organiser dans la ville un dispositif de distribution et de récupération de seringues: Une action de terrain efficace pour la prévention du sida et des hépatites chez les usagers de drogues par voie intraveineuse, Paris, juin 1999, p. 5. (Collection VIH/sida).

11. Françoise BOUCHARD, Marie GOURDEAU et Jacinthe GAGNON. "La séroconversion d'un travail-leur : impacts cliniques et économiques », Objectif prévention, ASSTSAS, vol. 25, $\mathrm{n}^{\circ} 2,2002$, p. 4. 
Les autres utilisateurs d'aiguilles à des fins professionnelles dans des domaines tels que l'acupuncture, la thanatopraxie, l'électrolyse, le tatouage et le perçage sont probablement exposés à des risques semblables (ou moindres dans le cas d'aiguilles non creuses) lorsqu'une blessure est causée par un instrument piquant ou tranchant sur lequel se trouve du sang frais.

On peut supposer que les piqûres accidentelles qui surviennent chez d'autres types de travailleurs (concierges, agents de sécurité, éboueurs, préposés à l'entretien des parcs, etc.) présentent généralement moins de risques infectieux que celles qui se produisent dans les milieux de soins car, normalement, il s'est écoulé un certain délai entre le moment où la seringue a été utilisée et celui où l'accident survient. Deux cas de contamination par le VIH ont été rapportés en France : l'un chez un éboueur et l'autre chez un collecteur de déchets ${ }^{12}$.

Dans le cas d'une piqûre avec une seringue abandonnée dans l'environnement, il est difficile de déterminer le niveau de risque étant donné que, entre autres choses, il est généralement impossible de déterminer le statut sérologique de la personne source et le temps pendant lequel la seringue a été laissée à cet endroit. Peu d'études ont été réalisées sur le sujet ${ }^{13}$. Toutefois, ceux qui se sont penchés sur la question s'accordent pour dire que le risque est faible, même s'il ne peut être considéré comme nul. Dans les faits, il n'existe aucun cas documenté de séroconversion pour l'infection par le VIH à la suite d'un incident avec une seringue abandonnée dans l'environnement au Canada, aux États-Unis et en Europe ${ }^{14}$. Dans une lettre publiée dans The Pediatric Infectious Disease Journal, un médecin rapporte toutefois le cas d'un enfant atteint d'une hépatite $\mathrm{B}$ aiguë dont le seul facteur d'exposition connu aurait été une blessure avec la seringue d'un voisin connu pour son infection par le VHB et par le VIH-1. Cet enfant n'avait reçu aucun traitement d'immunoprophylaxie ${ }^{15}$.

Dans ce contexte, les risques infectieux liés à chaque accident doivent être évalués afin de déterminer les mesures de prévention et de suivi appropriées (prophylaxie médicamenteuse et/ou vaccinale, surveillance biologique et sérologique, etc.). Un groupe de travail du MSSS procède actuellement à la révision des recommandations concernant la prophylaxie postexposition au sang et aux liquides biologiques ${ }^{16}$.

Par ailleurs, il faut rappeler que les piqûres d'aiguilles accidentelles peuvent aussi causer des infections locales ou systémiques analogues à celles des blessures avec des clous, qui exigent un traitement approprié. Pour toutes ces raisons, des précautions doivent être prises pour éviter ce type d'accidents.

12. MINISTÈRE DE LA SANTÉ ET DE LA PROTECTION SOCIALE (France) [en ligne]. [http:www.sante.gouv.fr/htm/actu/36_vih17.htm] (25 octobre 2004).

13. L'Institut national de santé publique du Québec procède actuellement à une revue de la littérature scientifique portant sur les risques de transmission d'infections liés à la présence de seringues et d'aiguilles à des endroits inappropriés. Les résultats de cet exercice pourront apporter un éclairage complémentaire à la réflexion sommaire présentée ici.

14. BC CENTRE FOR EXCELLENCE ON HIV/AIDS, [en ligne]. [http://www.cfenet.ubc.ca/guide/page/sectg/consg2.html] (25 octobre 2004).

15. Oscar GARCIA-ALGAR, M.D. «Hepatitis B virus infection from a needle stick », The Pediatric Infectious Disease Journal, Vol. 16(11), novembre 1977, p. 1099, [en ligne]. [http://gateway.ut.ovid.com/gw1/ovidweb.cgi].

16. Comme mesure postexposition, il est possible, selon le cas, d'offrir la vaccination et l'administration d'immunoglobulines pour prévenir l'hépatite B. Dans le cas de l'infection par le VIH, le BC Centre for Excellence on HIV/AIDS ne recommande pas de traitement antirétroviral à la suite d'une exposition accidentelle à des seringues abandonnées en dehors des milieux de soins lorsque l'origine de la seringue et le temps d'abandon sont inconnus. Outre la surveillance sérologique, il n'y a pas d'autre recommandation pour la prophylaxie concernant l'infection par le VHC. 


\subsection{Les conséquences d'une élimination non sécuritaire des seringues et des aiguilles pour certains services publics}

Les pratiques inappropriées en matière d'élimination des seringues et des aiguilles forcent les organismes et les entreprises qui en subissent les conséquences à mettre en place diverses mesures visant à protéger leur personnel et les usagers de leurs services. En plus de créer une inquiétude chez les travailleurs, ces mesures coûtent du temps et de l'argent aux employeurs et, par ricochet, à toute la société.

\subsubsection{Les services de garde à l'enfance}

Les seringues usagées constituent une préoccupation importante pour les centres de la petite enfance, particulièrement pour ceux du centre-ville de Montréal. Un exemple : depuis 2000, le personnel de La Sourithèque (qui accueille 140 enfants) trouve chaque année une trentaine de seringues à des endroits fréquentés par les enfants. Au moins une éducatrice s'est piquée accidentellement.

Les responsables de services de garde que nous avons consultés nous ont fait part de diverses précautions prises afin de protéger les enfants et le personnel.

- On inspecte la cour chaque jour avant d'y laisser jouer les enfants.

- On ratisse les carrés de sable.

- Avant d'amener les enfants au parc, on procède à une inspection des lieux afin d'éliminer les seringues et tout autre objet pouvant représenter un danger pour eux.

- Un parc public a été retiré de la liste des endroits où l'on amène les enfants.

- On sensibilise les enfants : s'ils trouvent une seringue, ils ne doivent jamais y toucher et ils doivent prévenir un adulte ${ }^{17}$.

- Les éducatrices ont en permanence dans leur sac à dos un contenant dans lequel elles pourront déposer les seringues trouvées éventuellement.

- On a éliminé les objets tels que contenants de peinture et de colle en forme de seringue pour éviter que les seringues deviennent des objets familiers pour les enfants.

\subsubsection{Les établissements d'enseignement}

Des établissements d'enseignement, particulièrement au centre-ville de Montréal, trouvent fréquemment des seringues usagées sur leurs terrains. Le cégep du Vieux-Montréal est de ceuxlà. On y a rapporté deux cas de piqûres accidentelles chez les employés. Afin d'éviter les accidents, un agent de sécurité procède chaque matin à une inspection des lieux. Il ramasse des seringues usagées presque tous les jours.

La Commission scolaire de Montréal ne tient pas de statistiques sur le nombre de seringues trouvées dans les écoles (il y en aurait peu), ni dans les cours d'école (il y en aurait davantage).

17. Le ministère de la Santé et des Services sociaux a produit des outils de sensibilisation à l'intention des enfants, des parents et des intervenants, soit une affiche dont le message est le suivant : " Si tu trouves des seringues, n'y touche pas, avertis un adulte » et un dépliant intitulé Prudence avec les seringues trouvées dans un lieu public. 
On rapporte souvent des cas de seringues trouvées par les enfants, surtout dans les ruelles, les parcs et les « recoins » qu'ils fréquentent. Des piqûres accidentelles sont survenues, mais on n'en connaît pas le nombre. La présence de seringues usagées est un phénomène récurrent, qui connaît des moments forts d'une durée de deux à trois semaines, nous dit-on. Il n'existe pas de politique réseau, c'est-à-dire s'appliquant à tout le territoire, face à ce problème. On agit plutôt localement. Par exemple, lorsqu'il y a recrudescence du phénomène, l'infirmière scolaire est appelée à l'aide. Elle donne alors des conseils préventifs aux élèves et au personnel de l'école.

L'Université du Québec à Montréal (UQAM), qui est située dans un secteur fréquenté par les UDI, a installé trois collecteurs extérieurs destinés à la récupération des seringues usagées. On déplore que ces collecteurs soient parfois vandalisés. Outre les seringues récupérées dans les collecteurs extérieurs, l'UQAM a ramassé, entre le 2 novembre 2002 et le 9 juin 2004, 769 seringues éparpillées sur ses terrains et dans ses locaux.

\subsubsection{Les municipalités}

Certains travailleurs des services municipaux sont particulièrement exposés au risque de piqûres accidentelles, notamment ceux qui sont affectés à l'entretien des parcs et à la collecte des ordures. Des usagers d'équipements collectifs tels que parcs, stationnements et toilettes publiques sont parfois victimes de tels accidents. Même les seringues déposées dans des contenants considérés comme résistants à la perforation peuvent causer des accidents lorsque ces contenants sont jetés aux ordures ménagères. Par exemple, si le contenant est compressé, les seringues peuvent le perforer ; s'il est jeté dans la chute à déchets d'un immeuble de plusieurs étages, le couvercle peut se détacher; des contenants sont parfois éventrés par l'équipement de déneigement et leur contenu, dispersé.

Les services municipaux du seul arrondissement Ville-Marie (centre-ville de Montréal) ramassent, depuis 2002, entre 6000 et 9000 seringues par année. Deux employés ont été victimes de piqûres accidentelles ces dernières années. La Ville de Montréal a installé sept collecteurs de seringues (voir illustration à l'annexe II) dans des parcs publics. Cinq de ces collecteurs se trouvent dans l'arrondissement Ville-Marie. Quelques-uns sont remplis et doivent être remplacés deux ou trois fois par semaine.

Les collecteurs extérieurs ne règlent pas tous les problèmes. En effet, ces contenants sont parfois vandalisés « pas nécessairement parce que des UDI veulent se procurer des seringues (ce qu'ils pourraient obtenir gratuitement à CACTUS-Montréal ou à Spectre de rue), mais parce qu'ils croient que certaines seringues usagées pourraient contenir des traces de drogue, affirment des représentants de l'arrondissement. De plus, certaines personnes, lorsqu'elles sont intoxiquées, ne sont pas en mesure de déposer les seringues dans l'ouverture prévue à cette fin. Des seringues se retrouvent donc parfois sur le sol aux alentours du collecteur. »

À Trois-Rivières, des employés municipaux font le tour des «points chauds » chaque lundi matin. En collaboration avec la Direction de santé publique, de la formation a été donnée aux employés le plus directement concernés, soit ceux qui sont affectés à l'entretien des parcs et au ramassage des ordures.

La Ville de Sherbrooke a élaboré une procédure d' "élimination sécuritaire des seringues et des aiguilles" à l'intention du personnel de l'entretien des parcs, des serres municipales, de l'arboriculture, de la collecte des déchets et à l'intention des équipes de la voirie. Cette procédure décrit les mesures à prendre pour éviter les piqûres accidentelles, la façon de se débarrasser des seringues souillées et la démarche à suivre en cas de lésion. 


\subsubsection{Postes Canada}

Postes Canada ne dispose pas de statistiques sur le nombre de seringues trouvées dans le courrier. On déplore cependant, depuis 1999, trois accidents impliquant des seringues ou des aiguilles. Un seul de ces accidents est survenu au Québec, à Montréal. La Société a mis au point une procédure visant à prévenir les blessures, une marche à suivre en cas de blessure causée par une seringue et des consignes quant à la façon de se débarrasser d'une seringue trouvée.

Pour prévenir les blessures, la politique adoptée prévoit que la Société mette à la disposition des employés les plus exposés des gants de caoutchouc à utiliser pour la levée des boîtes aux lettres publiques et au moment d'étendre le courrier sur les courroies de séparation. Des bouteilles de plastique jetables devraient être placées dans les véhicules et dans l'aire de tri pour y déposer les seringues qui seront éventuellement trouvées ${ }^{18}$.

\subsubsection{Hydro-Québec}

À Hydro-Québec, ce sont les travailleurs qui descendent dans les puits d'accès, surtout à Montréal et à Québec, qui sont le plus exposés aux piqûres accidentelles. Jusqu'à présent, on ne déplore aucun accident chez ce groupe d'employés, mais un programme de formation a été élaboré à leur intention. On leur donne de l'information sur les risques d'infection et des consignes sur la manipulation sécuritaire des seringues trouvées. Les camions sont équipés de contenants rigides pour DBM piquants et tranchants.

\subsubsection{Les services préhospitaliers d'urgence}

À la Corporation d'urgences-santé, qui offre les services préhospitaliers d'urgence sur les territoires de Montréal et de Laval, on déplore chaque année des piqûres accidentelles chez le personnel. De 1997 à septembre 2004, 53 accidents de ce genre sont survenus. Au début de cette période, plusieurs accidents étaient dus à des erreurs dans la manipulation et l'élimination des seringues et des aiguilles (p. ex. recapuchonnage). Les employés ont été sensibilisés à ces questions. Leur trousse d'urgence comprend maintenant un petit contenant pour DBM. Les ambulances sont aussi équipées de contenants pour DBM piquants et tranchants. Le tout a entraîné une diminution importante du nombre de piqûres accidentelles (4 en 2003 contre 10 en 2000). À présent, la grande majorité de ces accidents se produisent au domicile du patient. Les objets en cause sont des seringues utilisées pour des autosoins (p. ex. injection d'insuline ou d'épinéphrine).

18. POSTES CANADA, Sécurité : Seringues hypodermiques trouvées dans le courrier, Système du manuel de la Société (2002.09), $\mathrm{n}^{\mathrm{o}}$ 1605.15. 


\section{L'utilisation de seringues et d'aiguilles}

La mise en place d'un système d'élimination sécuritaire des seringues et des aiguilles usagées suppose d'abord une connaissance des différents contextes où ces instruments sont utilisés. La recherche de moyens appropriés exige une estimation des quantités de seringues et d'aiguilles en cause, selon les types d'utilisation.

\subsection{Les contextes d'utilisation d'instruments piquants et tranchants}

Lorsqu'il est question de seringues et d'aiguilles, on pense spontanément aux soins donnés dans les hôpitaux, les CLSC et les CHSLD ainsi que dans les cliniques médicales et dentaires. Cependant, plusieurs autres activités, à l'extérieur des milieux de soins de santé traditionnels, requièrent l'usage d'instruments piquants et tranchants (aiguilles, seringues, papillons, bistouris, lancettes, etc.).

Les campagnes de vaccination, par exemple, se déroulent souvent dans les écoles ou dans les milieux de travail. Dans le contexte du virage ambulatoire, de plus en plus de soins sont donnés à domicile par des professionnels de la santé. Plusieurs de ces soins (p. ex. ponctions veineuses, administration d'anticoagulants ou d'analgésiques, traitements contre le cancer, débridage de plaies, etc.) exigent l'emploi de seringues, d'aiguilles, de papillons, de bistouris.

La pratique des autosoins, qui a été longtemps associée principalement aux tests de glycémie et à l'injection d'insuline chez les diabétiques, est de plus en plus répandue. La personne elle-même (ou un proche) est appelée à utiliser des seringues ou des aiguilles à la maison dans diverses circonstances : traitement de maladies auto-immunes telles que la sclérose en plaques, dialyse péritonéale dans le cas d'insuffisance rénale, administration d'antibiotiques, chimiothérapie, injection de narcotiques en soins palliatifs, etc.

Des instruments piquants et tranchants sont également utilisés dans les milieux suivants: acupuncture, thanatopraxie, médecine vétérinaire, laboratoires, centres de recherche, collectes de sang, enseignement des soins infirmiers, services médicaux des grandes entreprises, électrolyse, tatouage et perçage. Les usagers de drogues par injection et les consommateurs de diverses substances telles que les stéroïdes anabolisants utilisent aussi des seringues.

Tous ces instruments piquants et tranchants deviennent autant de déchets biomédicaux dont il faut se débarrasser de façon sécuritaire.

\subsection{Les quantités de seringues et d'aiguilles en cause}

Plusieurs dizaines de millions d'aiguilles et de seringues avec aiguilles sont vendues au Québec au cours d'une année. Toutes ces seringues et toutes ces aiguilles se transformeront tôt ou tard en déchets biomédicaux (DBM) à traiter et à éliminer

Pour déterminer les mesures à mettre en place, nous avons cherché à connaître les quantités approximatives de ce type de DBM produites dans les divers contextes d'utilisation. 


\subsubsection{Les établissements publics de santé}

Les établissements publics de santé ne tiennent pas une comptabilité distincte des seringues et des aiguilles usagées qui se trouvent parmi leurs DBM. Ils ne sont d'ailleurs aucunement tenus de le faire. De même, peu d'établissements ont des données spécifiques concernant les quantités de DBM piquants et tranchants, ces derniers étant compris dans le volume total des DBM non anatomiques produits. Quelques établissements qui ont participé à nos enquêtes étaient néanmoins en mesure de nous fournir cette information. Voici quelques exemples de quantités de DBM piquants et tranchants produites durant une année par divers types d'établissements, de tailles et de milieux différents :

- Hôpital Charles-Lemoyne, Greenfield Park (571 lits) : 24497 kg ;

- Institut de cardiologie de Montréal (150 lits) : $16870 \mathrm{~kg}$;

- Hôpital Sainte-Croix de Drummondville (191 lits) : 15998 kg ;

- CLSC des Faubourgs, au centre-ville de Montréal (qui compte trois points de service, offre des soins à domicile, procède à des campagnes de vaccination à l'extérieur et constitue un site d'échange de seringues pour les UDI) : $1282 \mathrm{~kg}$;

- CLSC de Pierrefonds, Montréal (qui offre des soins à domicile et tient des campagnes de vaccination dans les écoles) : $1058 \mathrm{~kg}$;

- À Matane, le CH (85 lits) : $2300 \mathrm{~kg}$; le CHSLD (106 lits) : $112 \mathrm{~kg}$; le CLSC : $240 \mathrm{~kg}$.

Dans ce dernier exemple, on voit que le $\mathrm{CH}$ produit grosso modo 10 fois plus de DBM piquants et tranchants que le CLSC et 20 fois plus que le CHSLD qui compte un plus grand nombre de lits. On constate également que les DBM piquants et tranchants produits par deux gros CLSC de Montréal représentent chacun environ la moitié de ce qui est produit par un petit hôpital, celui de Matane.

Des données datant du début des années 90 indiquent que les hôpitaux génèrent près de $85 \%$ des déchets biomédicaux au Québec. Aucune information obtenue au cours de nos consultations ne nous permet de croire que la proportion serait sensiblement différente aujourd'hui.

\subsubsection{Les autres utilisateurs professionnels}

Les quantités de DBM piquants et tranchants produites par les autres utilisateurs sont beaucoup moindres que celles des établissements publics de santé. En voici quelques exemples obtenus au cours de nos consultations.

- Trois cliniques de médecine familiale de tailles différentes produisent respectivement les quantités suivantes de DBM piquants et tranchants :

- 5 médecins (équivalent 1,9 à temps complet) : 30 litres $(6 \mathrm{~kg})$ par année ${ }^{19}$;

- 7 médecins et 2 infirmières : de 60 à $68 \mathrm{~kg}$ par année ;

- 12 médecins et 3 infirmières : de 175 à 250 litres (35 à $50 \mathrm{~kg}$ ) par année.

- Une entreprise de soins infirmiers privés produit deux caisses de 24 contenants de 4,5 litres chacun $(43,2 \mathrm{~kg})$ en une année.

- Les réponses fournies par 7 cliniques dentaires comptant entre deux et cinq dentistes chacune montrent qu'un dentiste produit en moyenne $5 \mathrm{~kg}$ de DBM piquants et tranchants par année.

19. Cinq litres de DBM piquants et tranchants correspondent à environ 1 kilogramme. 
- Les réponses de 6 thanatologues indiquent qu'ils produisent aussi de très petites quantités de DBM piquants et tranchants. L'unité de mesure variant d'un répondant à l'autre, voici quelques exemples de réponses : 1 livre, 2 kilos, 4,5 litres par année.

- Un acupuncteur utilise entre 10 et 20 aiguilles par traitement, soit approximativement 400 aiguilles par jour. Il mettra jusqu'à une année à remplir un contenant de 7,6 litres $(1,5 \mathrm{~kg}) \mathrm{de}$ DBM.

- Le plus gros salon de tatouage et perçage de Trois-Rivières remplira environ 12 contenants de 7,6 litres $(18 \mathrm{~kg})$ de DBM piquants et tranchants par année.

- Une électrolyste mettra une année, sinon davantage, à remplir un contenant de 0,5 litre de filaments à usage unique utilisés dans ses activités.

\subsubsection{Les autosoins}

De l'avis de tous les pharmaciens consultés, les diabétiques représentent la plus vaste clientèle utilisatrice de seringues et d'aiguilles. D'autres patients, dont les personnes atteintes de sclérose en plaques, font également usage de seringues pour les autosoins.

- Les diabétiques

Selon des données fournies par l'Institut national de santé publique du Québec et par l'association Diabète Québec, on trouverait au Québec environ 300000 personnes ayant un diagnostic de diabète. Dans $10 \%$ des cas, soit 30 000, il s'agit du diabète de type 1 . La survie de ces personnes dépend d'injections quotidiennes d'insuline. Parmi les diabétiques de type 2, 50000 s'injectent également de l'insuline. Au total, ce sont donc 80000 diabétiques qui ont recours à l'injection d'insuline. Le traitement à l'insuline augmente constamment puisque l'on recommande maintenant le recours à l'insuline à un stade plus précoce de l'évolution de la maladie, ce qui évite les complications ${ }^{20}$.

Pour évaluer le volume de seringues et d'aiguilles en cause dans les autosoins des diabétiques, nous avons cherché à connaître le nombre d'injections requises par les diabétiques, de même que la proportion de ces injections qui sont données au moyen de seringues conventionnelles, d'une part, et au moyen du stylo injecteur, d'autre part.

Un pharmacien qui compte plusieurs diabétiques parmi sa clientèle nous indique que, d'après son expérience, le nombre d'injections par jour est le suivant :

- 4 injections par jour : $60 \%$ des patients ;

- 2 injections par jour : $30 \%$ des patients ;

- 1 injection par jour : $10 \%$ des patients.

Ces données peuvent évidemment varier selon la condition des patients et selon les pratiques médicales du milieu.

Dans quelles proportions les diabétiques utilisent-ils les seringues conventionnelles comparativement au stylo injecteur? Nous avons posé cette question à des fabricants, à plusieurs pharmaciens et à deux intervenants dans une clinique du diabète. Selon l'expérience des uns et

20. Source : Centre régional du diabète de la Cité de la santé de Laval. 
des autres, les réponses variaient considérablement. De l'avis du plus grand nombre, $25 \%$ des diabétiques utilisent les seringues et $75 \%$ utilisent le stylo injecteur.

Cela permet d'estimer à 90520000 le nombre d'injections requises annuellement pour les autosoins des diabétiques. De ce nombre, 22630000 injections seraient données avec une seringue et 67890 000, avec un stylo injecteur. Le tableau 2 présente les calculs effectués.

\section{Tableau 2}

Nombre d'injections par jour et par année pour 80000 diabétiques

\begin{tabular}{|c|c|c|c|c|c|c|}
\hline $\begin{array}{l}\text { A } \\
\text { Nombre } \\
\text { d'injections } \\
\text { quotidiennes } \\
\text { selon } \\
\text { ordonnance }\end{array}$ & $\begin{array}{l}\text { B } \\
\text { Proportion } \\
\text { des } \\
\text { diabétiques }\end{array}$ & $\begin{array}{l}\text { C } \\
\text { Nombre de } \\
\text { personnes } \\
(80000 \times \text { B) }\end{array}$ & $\begin{array}{l}\text { D } \\
\text { Nombre } \\
\text { total } \\
\text { d'injections } \\
\text { par jour } \\
(\text { A x C) }\end{array}$ & $\begin{array}{l}\text { E } \\
\text { Nombre } \\
\text { total } \\
\text { d'injections } \\
\text { par année } \\
\text { (D x 365) }\end{array}$ & $\begin{array}{l}\text { F } \\
\text { Nombre } \\
\text { d'injections } \\
\text { par an avec } \\
\text { seringue } \\
\text { (E x 25\%) }\end{array}$ & $\begin{array}{l}\text { G } \\
\text { Nombre } \\
\text { d'injections } \\
\text { par an avec } \\
\text { stylo } \\
\text { injecteur } \\
\text { (E x } 75 \%)\end{array}$ \\
\hline 4 & $60 \%$ & 48000 & 192000 & & & \\
\hline 2 & $30 \%$ & 24000 & 48000 & & & \\
\hline 1 & $10 \%$ & 8000 & 8000 & & & \\
\hline TOTAL & $100 \%$ & 80000 & 248000 & 90520000 & 22630000 & 67890000 \\
\hline
\end{tabular}

Dans l'hypothèse où tous ces diabétiques respecteraient la consigne d'utiliser une nouvelle seringue ou une nouvelle aiguille à chaque injection ; en tenant compte du fait qu'un contenant réglementaire de 7,6 litres peut renfermer 300 seringues ou 1500 aiguilles ; dans la mesure où tous ces contenants seraient remplis à la limite recommandée ; et sachant qu'un kilogramme de DBM piquants et tranchants équivaut à environ 5 litres, il faudrait chaque année 120693 contenants de cette dimension (poids total : environ $183453 \mathrm{kilos}^{21}$ de DBM), pour récupérer de façon sécuritaire les seringues et les aiguilles de tous les diabétiques qui s'injectent de l'insuline au Québec. Les lancettes, qui sont de petit format, pourraient vraisemblablement être incluses dans ces quantités. Ces données sont, cela va sans dire, purement approximatives. Le tableau 3 présente le calcul effectué.

À cette quantité de DBM, il faudrait ajouter les lancettes utilisées par les autres diabétiques, soit 220000 personnes, pour les tests de glycémie. Sachant qu'une lancette requiert environ 6 fois moins d'espace qu'une seringue $1 \mathrm{cc}$, et que l'on peut déposer environ 60 seringues dans un contenant de 1 litre, un diabétique qui utilise une lancette par jour, aurait besoin d'environ un contenant de ce type par année $(60$ × $6=360)$.

15. Selon cet estimé, le poids total des DBM produits en une année par tous les diabétiques du Québec qui s'injectent de l'insuline représente moins de la moitié du poids des DBM non anatomiques produits par le CHUM, soit $372531 \mathrm{~kg}$. 
Tableau 3

Production annuelle totale de DBM provenant des autosoins requis pour les diabétiques qui s'injectent de l'insuline

\begin{tabular}{|c|c|c|c|c|c|}
\hline $\begin{array}{l}\text { Instrument } \\
\text { pour injection } \\
\text { d'insuline }\end{array}$ & $\begin{array}{l}\text { A } \\
\text { Nombre } \\
\text { d'injections } \\
\text { par année }\end{array}$ & $\begin{array}{l}\text { B } \\
\text { Quantité par } \\
\text { contenant de } \\
7,61\end{array}$ & $\begin{array}{l}\mathrm{C} \\
\mathrm{N}^{\text {bre }} \text { de } \\
\text { contenants de } \\
7,6 \text { I requis } \\
(\mathrm{A} \div \mathrm{B}) \\
\end{array}$ & $\begin{array}{l}D \\
N^{\text {bre }} \text { total de } \\
\text { litres } \\
(C \times 7,6)\end{array}$ & $\begin{array}{l}\text { E } \\
\text { Équivalent } \\
\text { en } \\
\text { kilogrammes } \\
(D \div 5)\end{array}$ \\
\hline $\begin{array}{l}\text { Seringues } \\
\left(\mathrm{n}^{\text {bre de }}\right. \\
\text { seringues } \\
\text { requises })\end{array}$ & 22630000 & 300 & 75433 & & \\
\hline $\begin{array}{l}\text { Stylo injecteur } \\
\left(\mathrm{n}^{\text {bre }} \text { d'aiguilles }\right. \\
\text { requises })\end{array}$ & 67890000 & 1500 & 45260 & & \\
\hline TOTAL & 90520000 & & 120693 & 917266 & 183453 \\
\hline
\end{tabular}

- Les personnes atteintes de sclérose en plaques

Selon la Société de la sclérose en plaques, il y aurait 12000 personnes atteintes de sclérose en plaques au Québec. "Mais, ce sont de vieilles statistiques», nous dit-on. Selon le cas, ces personnes peuvent recevoir un traitement intramusculaire qui exige une injection par semaine ou l'un de trois traitements sous-cutanés qui exigent soit une injection par 2 jours, 3 injections par semaine ou une injection quotidienne. Ne connaissant pas le nombre - même approximatif - de personnes qui ont recours à l'un ou l'autre de ces traitements, il est difficile d'estimer le nombre de seringues en cause. Chose certaine, la personne qui utilise le plus grand nombre de seringues, soit une par jour, n'en utilise que 365 par année. Il lui faudrait à peine plus d'un contenant de 7,61 par année pour les récupérer de façon sécuritaire.

\subsubsection{Les usagers de drogues par injection}

On estime à 23000 le nombre d'usagers de drogues par injection au Québec ${ }^{22}$. Durant la période d'avril 2002 à mars 2003, 1107130 seringues leur ont été distribuées dans les centres d'accès aux seringues. Ce nombre n'inclut pas les seringues vendues en pharmacie dont le nombre est estimé à 200000 à Montréal et à 23500 à Québec ${ }^{23}$. Nous ignorons les quantités de seringues vendues en pharmacie dans les autres régions. Au total, c'est un minimum de 1330630 seringues qui ont été mises à la disposition des UDI au cours de cette année.

22. R. REMIS, P. LECLERC et A. C. VANDAL. La situation du sida et de l'infection au VIH au Québec 1996, Direction de santé publique de Montréal-Centre pour le ministère de la Santé et des Services sociaux du Québec, 1999, p. A3-5, cité dans MINISTÈRE DE LA SANTÉ ET DES SERVICES SOCIAUX et INSTITUT NATIONAL DE SANTÉ PUBLIQUE DU QUÉBEC. Liste officielle des centres d'accès aux seringues du Québec (distribution, vente et récupération) 2003-2004: Programme de prévention de la transmission du VIH et des hépatites chez les utilisateurs de drogues par injection, p. 5.

23. Lina NOËL, Richard CLOUTIER et Marika MUNGER. Statistiques sur les services relatifs aux programmes de prévention du VIH et des hépatites B et C offerts aux utilisateurs de drogues par injection du Québec, avril 2002 à mars 2003, ministère de la Santé et des Services sociaux et Institut national de santé publique du Québec, mai 2004, p. 5-7. 
La quantité de seringues utilisées variant considérablement d'une personne à l'autre, il est impossible d'estimer le nombre de contenants nécessaires pour récupérer toutes ces seringues. Nous savons seulement qu'il en faudrait au moins 23000 au départ. Le nombre dépend également des formats de contenants qui leur seront fournis, ceux-ci pouvant varier de 5 à 300 seringues. 


\section{L'encadrement réglementaire}

La récupération et l'élimination des seringues et des aiguilles usagées sont encadrées par le Règlement sur les déchets biomédicaux adopté en $1992^{24}$. Dans le but de favoriser la récupération des seringues usagées de nature domestique, des assouplissements à l'application de ce règlement ont été apportés par les Lignes directrices sur la récupération des seringues usagées publiées par le ministère de l'Environnement et de la Faune en 1996.

\subsection{Le Règlement sur les déchets biomédicaux}

Le Règlement sur les déchets biomédicaux prescrit des normes d'entreposage, de transport, de traitement et d'élimination pour l'ensemble des déchets biomédicaux. Certaines dispositions de ce règlement s'appliquent plus particulièrement à l'élimination des seringues et des aiguilles usagées. Les extraits du Règlement sont en italique.

\subsubsection{Champ d'application}

Art. 1 - Le présent règlement s'applique aux déchets biomédicaux suivants : [...]

$3^{\circ}$ tout déchet non anatomique constitué de l'un des éléments suivants :

a) un objet piquant, tranchant ou cassable qui a été en contact avec du sang, un liquide ou un tissu biologique, provenant de soins médicaux, dentaires ou vétérinaires ou d'un laboratoire de biologie médicale ou vétérinaire ou un tel objet provenant de l'exercice de la thanatopraxie.

[...]

Certains usages professionnels de seringues et d'aiguilles ne semblent pas couverts par cette énumération, soit l'acupuncture, l'électrolyse, le tatouage et le perçage.

Art. 2 - Le présent règlement ne s'applique pas : [...]

$5^{\circ}$ aux déchets biomédicaux non anatomiques provenant d'activités domestiques.

Les seringues, les aiguilles et les lancettes utilisées pour les autosoins, tout comme les seringues utilisées par les usagers de drogues par injection, sont exclues de l'application du Règlement.

Art. 3 - L'article 14, le deuxième alinéa de l'article 15, les articles 25, 37 à 39, 45 à 51, 63 et 64 ne s'appliquent pas à l'exploitant d'un système de transport de déchets biomédicaux, s'il en transporte moins de 50 kilogrammes par mois.

Toutefois, cet exploitant n'est pas tenu de maintenir les déchets biomédicaux à une température inférieure à $4{ }^{\circ} \mathrm{C}$.

Les exigences relatives au registre quotidien, au rapport annuel, au certificat d'autorisation, au véhicule (réservé exclusivement à cet usage, affiche, réfrigération, cuvette de rétention, compartiments en matière non poreuse) et à la désinfection après le déchargement ne s'appliquent pas à ceux qui transportent moins de $50 \mathrm{~kg}$ de DBM par mois.

De plus, ceux qui transportent eux-mêmes ces petites quantités de DBM ne sont pas tenus de les réfrigérer durant l'entreposage, comme l'exige le $2^{\mathrm{e}}$ alinéa de l'article 22.

24. Ce règlement sera révisé au cours des prochains mois. 


\subsubsection{Gestion des DBM}

Art. 6 - Les déchets biomédicaux non anatomiques doivent être traités par désinfection ou incinération.

Art. 11 - Nul ne peut rejeter dans un réseau d'égout des déchets biomédicaux.

Art. 12 - L'exploitant d'un lieu de production de déchets biomédicaux doit tenir à jour un registre dans lequel il inscrit chaque semaine la nature et la quantité des déchets biomédicaux produits.

Cette exigence s'applique à tous les producteurs de DBM.

Art. 14 - L'exploitant d'un système de transport de déchets biomédicaux doit tenir un registre quotidien indiquant :

$1^{\circ}$ la nature des déchets biomédicaux transportés ;

$2^{\circ}$ leur quantité ;

$3^{\circ}$ l'adresse du lieu de leur provenance et de leur destination.

Cette exigence ne s'applique pas à ceux qui transportent moins de $50 \mathrm{~kg}$ de DBM par mois.

Art. 15 - L'exploitant d'un lieu de production de déchets biomédicaux doit, le premier avril de chaque année, préparer un rapport conformément à la formule prescrite à l'annexe I.

L'exploitant d'une installation de traitement de déchets biomédicaux, par désinfection ou incinération, hors du lieu de leur production, d'une installation d'entreposage de déchets biomédicaux hors du lieu de leur production ou d'un système de transport de déchets biomédicaux doit, à cette même date, préparer un rapport conformément à la formule prescrite à l'annexe II.

Ceux qui transportent moins de $50 \mathrm{~kg}$ de DBM par mois doivent produire un rapport annuel de production de DBM (formule de l'annexe I), mais sont exemptés du rapport annuel de gestion de DBM (formule de l'annexe II)

Art. 22 - Les déchets biomédicaux destinés à être expédiés hors du lieu de leur production doivent être déposés dans des contenants rigides, scellés et étanches. Ces contenants doivent de plus être résistants à la perforation s'ils contiennent des déchets biomédicaux visés au sousparagraphe a du paragraphe 3 de l'article 1.

Ils doivent être maintenus dans un lieu réfrigéré à une température inférieure à $4{ }^{\circ} \mathrm{C}$.

Les dispositions du $1^{\text {er }}$ alinéa s'appliquent à tous. On y précise notamment que les contenants de DBM piquants et tranchants doivent résister à la perforation. L'exigence de la réfrigération ne s'applique pas aux DBM devant être transportés dont la quantité est inférieure à $50 \mathrm{~kg}$ par mois.

Art. 24 - [...] Les déchets biomédicaux non anatomiques doivent être expédiés à un titulaire d'un certificat d'autorisation pour l'exploitation d'une installation de traitement, par désinfection ou incinération, ou d'entreposage de déchets biomédicaux.

Actuellement, au Québec, seuls les établissements de santé munis d'autoclaves et l'entreprise spécialisée Services médicaux Med-Tech sont autorisés à traiter des DBM. 
Art. 25 - Les déchets biomédicaux ne peuvent être remis qu'au titulaire d'un certificat d'autorisation pour l'exploitation d'un système de transport de déchets biomédicaux.

L'exigence de recourir à un transporteur autorisé ne s'applique pas à ceux qui transportent moins de $50 \mathrm{~kg}$ de DBM par mois.

Art. 37 - L'exploitant d'un système de transport de déchets biomédicaux doit réserver exclusivement au transport de déchets biomédicaux le véhicule, le conteneur ou le contenant utilisé à ces fins.

Art. 38 - Cet exploitant doit apposer, sur les côtés et à l'arrière du véhicule utilisé, une affiche conforme à celle de l'annexe IV reproduisant le symbole international.

Art. 39 - Cet exploitant doit munir le véhicule utilisé :

$1^{\circ}$ d'un système de réfrigération permettant de maintenir en tout temps, à une température inférieure à $4{ }^{\circ} \mathrm{C}$ les déchets biomédicaux qui y sont contenus ;

$2^{\circ}$ d'une cuvette de rétention permettant de recueillir les écoulements provenant de ces déchets ; $3^{\circ}$ de compartiments en matière non poreuse conçus de façon à faciliter leur nettoyage.

Toutes les exigences relatives au véhicule ne s'appliquent pas à ceux qui transportent moins de $50 \mathrm{~kg}$ de DBM par mois.

Art. 65 - Toute infraction à l'une des dispositions des articles 5 à 11, 21, 22, 24, 25, 28, 29, 31 à 33, 35 à 37, 39, 40, 43 à 45, 56 ou 64 rend le contrevenant passible :

$1^{\circ}$ dans le cas d'une personne physique, d'une amende d'au moins $5000 \$$ et d'au plus 25000 \$;

$2^{\circ}$ dans le cas d'une corporation, d'une amende d'au moins 20000 \$ et d'au plus $500000 \$$.

Ces amendes sont portées au double dans le cas de récidive.

Les infractions pouvant entraîner ces amendes sont les dérogations

- aux procédés autorisés pour le traitement des DBM (art. 5 à 7);

- au maintien des installations et équipements en bon état de fonctionnement (art. 8);

- à l'interdiction de compresser mécaniquement des DBM (art. 10) ;

- à l'interdiction de les rejeter dans un réseau d'égout (art. 11) ;

- à l'interdiction de les mettre en contact avec d'autres types de matières résiduelles (art. 21);

- aux dispositions concernant les contenants requis et la réfrigération des DBM destinés à être expédiés hors du lieu de leur production (art. 24);

- à l'obligation de remettre les DBM uniquement à un transporteur autorisé (art. 25) ;

- aux exigences concernant l'aménagement des lieux d'entreposage et de traitement des DBM (art. 28) ainsi que des installations de nettoyage des contenants, des conteneurs et des véhicules (art. 29);

- à l'obligation de décharger directement les DBM du véhicule de transport au bâtiment destiné à leur entreposage ou à leur traitement (art. 31);

- à l'obligation pour l'exploitant d'une installation de traitement ou d'entreposage des DBM de refuser les déchets si les conditions relatives à la compression, aux contenants, à la réfrigération et aux étiquettes d'identification ne sont pas respectées (art. 32);

- à l'exigence de maintenir les DBM à une température de $4{ }^{\circ} \mathrm{C}$ (art. 33) ; 
- aux mesures prescrites en cas de fermeture temporaire ou définitive d'une installation de traitement ou d'entreposage de DBM (art. 35 et 36) ;

- à l'obligation de réserver exclusivement au transport de DBM le véhicule et le conteneur (art. 37) ;

- aux dispositions relatives à l'aménagement du véhicule (réfrigération, cuvette de rétention, compartiments en matière non poreuse) (art. 39) ;

- à l'obligation pour le transporteur de refuser les DBM si les conditions relatives à la compression, aux contenants, à la réfrigération et aux étiquettes d'identification ne sont pas respectées (art. 40) ;

- à l'interdiction de déplacer les DBM d'un véhicule à l'autre à moins d'un bris susceptible d'entraîner un déversement des DBM ou d'une substance en provenant (art. 43) ;

- aux mesures prévues en cas de déversement (art. 44);

- à l'obligation de désinfecter le véhicule et les conteneurs après le déchargement (art. 45) ;

- aux dispositions relatives à la garantie exigée de l'exploitant d'un incinérateur pour DBM (art. 56 et 64).

Toutefois, comme le règlement ne s'applique pas aux DBM non anatomiques provenant d'activités domestiques, la dérogation à l'une ou l'autre de ces dispositions (lorsque applicable) par les utilisateurs de seringues pour les autosoins ou pour la consommation de drogues ne peut entraîner aucune sanction.

\subsection{Les Lignes directrices sur la récupération des seringues usagées}

Les Lignes directrices sur la récupération des seringues usagées ont été formulées par le ministère de l'Environnement dans le but de faciliter la réalisation des programmes de récupération de seringues usagées de nature domestique, "c'est-à-dire utilisées par les consommateurs de drogues par injection, les diabétiques ou employées à des fins domestiques ».

On y précise que :

- les seringues usagées sont des déchets biomédicaux non anatomiques de la catégorie des objets piquants ou tranchants ;

- l'activité de récupération de seringues usagées est assimilée à une activité de production de DBM ;

- sont visés tous les établissements privés ou publics qui distribuent et récupèrent des seringues : pharmacies, cliniques médicales, centres spécialisés du réseau privé ou communautaire, CLSC, hôpitaux ou autres qui mettent à la disposition de leurs clients ou de leurs patients des contenants pour la récupération des seringues usagées de nature domestique ;

- seul le lieu initial de récupération (p. ex. pharmacie) est visé par ces lignes directrices. Les destinataires des seringues usagées ainsi récupérées (p. ex. $\mathrm{CH}$ muni d'autoclave) sont soumis à l'ensemble du Règlement sur les déchets biomédicaux ;

- le registre de production (art. 12 du Règlement) sera tenu au rythme de remplissage des contenants plutôt que de façon hebdomadaire. Il pourra aussi être remplacé par la facturation du transporteur ou du destinataire ;

- on devra prévoir un système de verrouillage pour les contenants de récupération des seringues rendant leur enlèvement impossible par quiconque n'y est pas autorisé ; 
- l'exigence de réfrigération des DBM (art. 22 du Règlement) ne s'applique pas aux programmes de récupération de seringues usagées ;

- si la quantité à transporter chaque mois est inférieure à $50 \mathrm{~kg}$, l'expéditeur peut effectuer le transport lui-même, sans permis d'exploitation à cette fin et sans devoir satisfaire aux exigences relatives au registre quotidien, au rapport annuel, au certificat d'autorisation, au véhicule (réservé exclusivement à cet usage, affiche, réfrigération, cuvette de rétention, compartiments en matière non poreuse) et à la désinfection après le déchargement. 



\section{Les procédés utilisés pour le traitement et l'élimination des déchets biomédicaux}

Suivant le Règlement sur les déchets biomédicaux (art. 6), les DBM non anatomiques doivent être traités par désinfection ou incinération. Actuellement, le procédé utilisé au Québec pour le traitement de la plupart des DBM non anatomiques, incluant les objets piquants et tranchants, est la décontamination thermique à la vapeur, puis l'enfouissement sanitaire.

Le groupe de travail n'a pas remis en question les choix de procédés arrêtés au début des années 1990 par le ministère de l'Environnement, en collaboration avec le ministère de la Santé et des Services sociaux, le Laboratoire de santé publique du Québec et l'Association des hôpitaux du Québec.

\subsection{La décontamination par la vapeur}

La décontamination des DBM par la vapeur se fait à l'aide d'un stérilisateur (autoclave) ${ }^{25}$. Le procédé, considéré comme assez simple, consiste à injecter de la vapeur à l'intérieur d'une enceinte fermée hermétiquement. La combinaison d'une température $\left(132{ }^{\circ} \mathrm{C}\right)$ et d'une pression $\left(30 \mathrm{psi}^{26}\right)$ pendant une durée donnée (90 minutes) tue les micro-organismes contaminants, éliminant ainsi les risques infectieux des déchets pour les manipulations ultérieures.

Un indicateur chimique inclus dans chaque charge à décontaminer indique que la charge a atteint la température recommandée. L'efficacité de la décontamination est vérifiée régulièrement au moyen d'indicateurs biologiques.

On trouve des autoclaves dans une soixantaine d'établissements à travers le Québec, surtout des hôpitaux, mais également quelques anciens hôpitaux dont la vocation a changé. L'entreprise spécialisée Services médicaux Med-Tech est aussi équipée de ce type d'appareil.

Quelques mots au sujet de Med-Tech : il s'agit d'une filiale de Stericycle, la seconde entreprise en importance dans ce domaine aux États-Unis. Cette entreprise dessert le marché québécois depuis plus de 10 ans. En faisant l'acquisition de Pyroval en 2002, elle est devenue la seule entreprise privée au Québec à offrir le service de décontamination et d'élimination des DBM non anatomiques à ceux qui n'ont pas accès à l'autoclave d'un établissement public.

\subsection{L’incinération}

En 1990, la compagnie DECOM exploitait un incinérateur pour déchets biomédicaux à Gatineau. De 15 à 20 incinérateurs étaient aussi en activité dans les hôpitaux québécois ${ }^{27}$. Les exigences plus sévères imposées par le Règlement sur les déchets biomédicaux et par le Règlement sur la qualité de l'atmosphère ont entraîné la fermeture de tous ces incinérateurs, à l'exception de celui

25. Voir illustration à l'annexe III.

26. Unité de pression en livres par pouce carré (pound per square inch).

27. COMMISSION D'ENQUÊTE SUR LES DÉCHETS DANGEREUX, Les déchets dangereux au Québec : une gestion environnementale, Québec, Bureau d'audiences publiques sur l'environnement, 1990, p. 326. 
de l'hôpital Notre-Dame, à Montréal. Par la suite, cet appareil a été jugé non rentable et il n'est plus utilisé depuis 1993.

Les DBM qui doivent être incinérés (déchets anatomiques humains et animaux, cytotoxiques et pharmaceutiques) sont normalement confiés à l'entreprise spécialisée Med-Tech qui les transporte à l'un des sites de la compagnie Stericycle aux États-Unis.

\subsection{L'enfouissement sanitaire}

Après avoir été décontaminés, les DBM peuvent être acheminés aux sites d'enfouissement sanitaire comme les déchets généraux. Selon les renseignements que nous avons pu obtenir, les sites d'enfouissement sanitaire accordent une attention spéciale aux déchets provenant des hôpitaux : ces déchets sont recouverts en priorité. Lorsqu'ils comprennent des objets piquants et tranchants, on pratique une tranchée spéciale dans laquelle ces déchets sont déversés, puis on recouvre et on compacte le tout immédiatement. 


\section{Les pratiques de récupération et d'élimination des seringues et des aiguilles usagées chez les différents types d'utilisateurs}

Après avoir évalué les quantités de DBM produites par les différents utilisateurs d'instruments piquants et tranchants, après avoir considéré l'encadrement réglementaire et les équipements disponibles, il convient d'examiner les pratiques de récupération et d'élimination des seringues et des aiguilles usagées qui ont cours chez ceux qui utilisent ces instruments, tant à des fins professionnelles que sur une base domestique.

\subsection{Les centres hospitaliers}

Notre enquête auprès des centres hospitaliers ne portait pas sur la façon dont les centres hospitaliers organisent l'ensemble du processus de récupération des DBM à l'intérieur de l'établissement (nous présumons qu'ils le font correctement), mais plutôt sur l'équipement dont ils disposent pour les traiter et les éliminer, de même que sur les collaborations établies avec d'autres producteurs de DBM. Au total, 85 établissements avec mission $\mathrm{CH}$ sur un total possible de 110 ont répondu au questionnaire.

Nous souhaitons toutefois attirer l'attention sur un élément du processus. Même si aucune question ne portait sur cet aspect, plusieurs responsables de services d'entretien sanitaire nous ont signalé des problèmes quant au tri des déchets à la source. Des déchets d'autre nature se retrouvent parfois dans les contenants prévus pour les DBM, ce qui a pour effet d'accroître inutilement le volume de DBM à traiter et à éliminer, augmentant d'autant le nombre de contenants réglementaires requis et le coût du traitement et de l'élimination des DBM.

\subsubsection{Les $\mathrm{CH}$ munis d'autoclaves}

Une soixantaine d'établissements à travers le Québec, généralement des hôpitaux, sont munis d'autoclaves pour la décontamination de leurs propres $\mathrm{DBM}^{28}$. La plupart d'entre eux traitent aussi des DBM provenant de l'extérieur. Après décontamination, ces déchets peuvent être ajoutés aux déchets généraux et acheminés à un site d'enfouissement sanitaire. Ils pourraient aussi être détruits dans un incinérateur municipal puisqu'ils ne sont plus considérés comme des DBM.

Parmi les $45 \mathrm{CH}$ munis d'autoclaves qui ont participé à notre enquête, 36 traitent des DBM produits par d'autres missions du même établissement et 29 traitent des DBM produits par d'autres établissements publics de santé. Parmi ces $29 \mathrm{CH}$, la moitié (14) offrent le service sans frais, tandis que les autres (15) exigent un paiement.

Plusieurs $\mathrm{CH}$ équipés d'autoclaves acceptent les DBM provenant de services privés (cliniques médicales, dentistes, acupuncteurs, thanatologues, etc.) de même que des organismes qui font la récupération de seringues auprès des individus (diabétiques, UDI et autres).

28. On trouve des autoclaves pour la décontamination des DBM dans toutes les régions du Québec à l'exception de Montréal, Laval, Québec et Chaudière-Appalaches. Nous reviendrons plus loin sur leur localisation précise. 
Les $\mathrm{CH}$ qui offrent le service à une ou plusieurs pharmacies se répartissent ainsi : 8 offrent le service sans frais ; 7 exigent un paiement. Aux organismes communautaires qui font la récupération de seringues, les $\mathrm{CH}$ sont deux fois plus nombreux à offrir ce service sans frais (8) qu'avec paiement (4).

Parmi la trentaine de $\mathrm{CH}$ qui offrent le même service aux cabinets privés (p. ex. cliniques médicales, dentistes, acupuncteurs, thanatologues et vétérinaires), ceux qui le font sans frais sont nettement moins nombreux que ceux qui exigent un paiement. La proportion varie selon les types de clients. Certains $\mathrm{CH}$ réclament un montant annuel. D'autres facturent les DBM au kilogramme seulement. Les tarifs fixés varient entre 1,50 et $5 \$$ le kilogramme.

Les $\mathrm{CH}$ qui traitent et éliminent les seringues usagées récupérées auprès des individus (surtout les UDI et les diabétiques) le font sans frais. Ils exigent généralement que ces personnes apportent leurs seringues à la pharmacie ou au CLSC, auxquels ils offrent le service de décontamination et d'élimination gratuitement.

\subsubsection{Les $\mathrm{CH}$ qui ne sont pas munis d'autoclaves}

Parmi les $\mathrm{CH}$ qui ont répondu à notre enquête, 40 ne sont pas munis d'autoclaves. Ils confient leurs DBM non anatomiques à Services médicaux Med-Tech, qui les décontamine au moyen de son autoclave, à Sainte-Catherine, sur la rive sud de Montréal, puis les achemine à un site d'enfouissement, tout comme le font les hôpitaux équipés d'autoclaves.

Cela va sans dire que les établissements qui doivent faire traiter leurs propres DBM à l'extérieur sont beaucoup moins enclins à accepter ceux des autres, y compris les seringues et les aiguilles récupérées auprès des individus (diabétiques, dialysés, UDI, etc.) que ceux qui sont dotés d'un autoclave. Une dizaine d'hôpitaux qui ne disposent pas d'autoclave ont néanmoins dit qu'ils accepteraient de recevoir - généralement avec paiement - des seringues provenant de l'extérieur.

\subsection{Les autres établissements publics de santé}

Parmi les 100 établissements avec mission CLSC qui ont participé à notre enquête auprès des CLSC et CHSLD, 38 ont indiqué que les seringues ${ }^{29}$ récupérées et éliminées par leur établissement provenaient uniquement des soins donnés par leur personnel. Une dizaine d'établissements récupèrent des seringues provenant des organismes communautaires et un nombre équivalent d'établissements récupèrent des seringues utilisées par des individus, diabétiques ou autres. Pour ce qui est des 28 établissements sans mission CLSC, sauf de rares exceptions, ils ne récupèrent que les seringues utilisées par leur personnel soignant.

Certains établissements qui refusent les seringues et les aiguilles provenant de l'extérieur ont motivé leur position de la façon suivante :

- service déjà offert par une autre mission de l'établissement ;

- service offert par un autre établissement ;

- service offert par les pharmaciens ;

- absence de politique locale à ce sujet ;

29. Notre question faisait mention uniquement des seringues et non pas des autres objets piquants ou tranchants tels que les aiguilles. 
- obligation de recourir à un autre établissement ou à une entreprise spécialisée pour le traitement et l'élimination de nos propres DBM ;

- manque d'espace pour l'entreposage ;

- manque d'espace pour de nouveaux congélateurs ;

- chambre réfrigérée trop exiguë ;

- manque de ressources humaines et financières ;

- absence d'équipement pour traiter les DBM (autoclave) ;

- limite fixée dans la réglementation concernant la quantité maximale de DBM pouvant être transportée sans le recours à une entreprise spécialisée (ce qui serait beaucoup plus coûteux) ;

- peur d'être ciblé comme lieu d'échange de seringues, crainte de dégradation de la qualité de vie.

En règle générale, les CLSC et les CHSLD acheminent leurs DBM aux hôpitaux équipés d'autoclave, lorsqu'il s'en trouve dans leur milieu. Les autres doivent recourir aux services de Med-Tech. Ils bénéficient toutefois des tarifs négociés par les services d'approvisionnement en commun.

La vaste majorité des CLSC et CHSLD qui ont participé à notre enquête ont indiqué que leur personnel qui offre des soins à domicile ou à l'extérieur de l'établissement reçoit les consignes suivantes :

- ne jamais replacer le capuchon sur l'aiguille ;

- déposer les seringues et les aiguilles dans des contenants pour DBM ;

- rapporter tous les objets piquants ou tranchants.

En ce qui a trait aux consignes données par le personnel de l'établissement (CLSC essentiellement) aux personnes qui utilisent des seringues à domicile pour des autosoins, on observe une très grande variété. La consigne de ne jamais replacer le capuchon sur l'aiguille est très répandue (68 établissements sur une centaine). Les consignes indiquées ci-après sont aussi mentionnées :

- déposer les seringues et les aiguilles dans un contenant en plastique ou en métal muni d'un couvercle et rapporter le contenant à la pharmacie (59);

- les déposer dans un contenant en plastique ou en métal muni d'un couvercle et rapporter le contenant au CLSC (30), soit deux fois moins qu'à la pharmacie ;

- les déposer dans un contenant pour DBM et rapporter le contenant au CLSC (43);

- les déposer dans un contenant pour DBM et rapporter le contenant à la pharmacie (36) ;

- les déposer dans un contenant en plastique ou en métal muni d'un couvercle et jeter le tout avec les déchets domestiques (36) ;

- utiliser le coupe-aiguilles (20).

Plusieurs répondants indiquent un ordre de priorité (p. ex. si la pharmacie refuse de récupérer les seringues, apporter le contenant au CLSC ou le jeter avec les déchets domestiques). 


\subsection{Les cliniques spécialisées}

Nous avons consulté deux cliniques spécialisées dans le traitement de maladies qui exigent des autosoins requérant l'usage de seringues et d'aiguilles. L'une d'elles traite le diabète ; l'autre, la sclérose en plaques.

La clinique spécialisée dans le traitement du diabète encourage l'utilisation du stylo injecteur. On juge ce moyen plus pratique que la seringue conventionnelle (qui exige que l'on remplisse d'abord la seringue d'insuline). Le stylo injecteur contient la cartouche d'insuline ; il suffit de tourner le bouton pour obtenir la quantité nécessaire. "Même des patients aveugles utilisent le stylo parce qu'ils peuvent entendre le déclic indiquant qu'ils ont la dose appropriée. » Il est recommandé d'utiliser une nouvelle aiguille à chaque injection.

Pour le moment du moins, il n'existe pas de dispositif tel que le stylo injecteur pour l'administration de médicaments autres que l'insuline. Les personnes atteintes de sclérose en plaques, par exemple, doivent donc recourir aux seringues conventionnelles.

Les deux cliniques spécialisées ne récupèrent pas les seringues et les aiguilles usagées de leurs patients. On invoque

- le manque d'espace : « Nous serions enterrés de boîtes. »

- les coûts : « Il y a des coûts associés à cette récupération. »

- et le manque de ressources : «Nous manquons de personnel, y compris pour la gestion des dossiers des patients. »

En ce qui a trait à l'élimination des aiguilles et des seringues souillées, la clinique du diabète recommande à ses patients de :

- ne jamais jeter les seringues et les aiguilles à la poubelle ;

- les déposer dans un contenant de plastique avec une petite ouverture ayant un couvercle qui se visse (p. ex. une bouteille d'eau de Javel) ;

- ne pas utiliser un contenant de verre ;

- demander à leur pharmacien ou à leur professionnel de la santé quelles sont les recommandations pour l'élimination des déchets dans leur région ${ }^{30}$.

Jusqu'à maintenant, cette clinique a encouragé l'usage de contenants opaques : «C'est plus discret. La personne risque moins d'être étiquetée comme malade et c'est moins attirant pour les enfants. »

La clinique de la sclérose en plaques recommande à ses patients de rapporter les contenants de seringues souillées à la pharmacie. Et si le pharmacien les refuse ? « Nous leur suggérons d'aller faire remplir leur ordonnance ailleurs. »

Il est à noter que ces deux cliniques spécialisées sont rattachées à de gros hôpitaux qui ne sont pas munis d'autoclaves.

30. CENTRE RÉGIONAL DU DIABÈTE, Technique d'injection: le stylo injecteur, Laval, Agence de développement de réseaux locaux de services de santé et de services sociaux de Laval, juin 2004, p. 6. 


\subsection{Les cliniques médicales privées}

L'information obtenue de six cliniques médicales privées indique que les pratiques y sont sensiblement les mêmes.

- Les DBM piquants et tranchants utilisés par les professionnels de la clinique sont déposés dans des contenants réglementaires. Toutes les personnes auxquelles nous avons posé la question ignoraient que, selon le règlement, ces DBM devaient être réfrigérés.

- On se débarrasse des DBM soit en les apportant à un hôpital de la région muni d'un autoclave, soit en les confiant à Med-Tech.

- Aucune clinique ne récupère les seringues et les aiguilles utilisées à domicile par ses patients. On leur suggère plutôt de les apporter à la pharmacie.

- Si une personne apportait à la clinique une ou quelques seringues trouvées dans l'environnement, on les accepterait et on les éliminerait avec les DBM de la clinique.

Rares sont les cliniques médicales qui participent aux programmes d'échange de seringues pour les UDI. En 2003-2004 on ne comptait que 3 cliniques médicales parmi les 700 centres d'accès aux seringues inscrites à la Liste officielle des centres d'accès aux seringues du Québec ${ }^{31}$.

Par ailleurs, même si l'on peut supposer que les cliniques médicales privées gèrent correctement leurs DBM, il faut signaler que sur 148 cas de "piqûres accidentelles dans la communauté », traités à l'hôpital Sainte-Justine de 1995 à 2003, 9 accidents sont survenus dans un cabinet privé de médecin ${ }^{32}$. Cela laisse croire qu'à certains endroits, des seringues et des aiguilles souillées ne sont pas placées hors de la portée des enfants.

\subsection{Les soins infirmiers privés}

Nous n'avons consulté qu'une seule entreprise de soins infirmiers privés. En plus d'offrir des soins et du soutien à domicile, cette entreprise procède notamment à des campagnes de vaccination dans les milieux de travail. Ses DBM piquants et tranchants sont déposés dans des contenants réglementaires.

Bien que située à proximité d'un hôpital muni d'un autoclave qui offre le service de décontamination des DBM à des clients de l'extérieur, cette entreprise recourt aux services de Med-Tech pour traiter et éliminer ses DBM.

\subsection{Les dentistes}

Les dentistes, comme les autres professionnels de la santé, recourent tantôt à un hôpital muni d'un autoclave, tantôt à Med-Tech, pour l'élimination de leurs DBM non anatomiques. Une troisième option leur est offerte : décontaminer quotidiennement ces DBM sur place (au moyen de l'autoclave servant à la stérilisation des instruments réutilisables) avant de les éliminer avec les déchets ordinaires. Les objets piquants et tranchants doivent toutefois être déposés dans des contenants rigides résistant aux perforations. Cette pratique est conforme à une entente intervenue

31. MINISTÈRE DE LA SANTÉ ET DES SERVICES SOCIAUX et INSTITUT NATIONAL DE SANTÉ PUBLIQUE DU QUÉBEC, Liste officielle des centres d'accès aux seringues du Québec (distribution, vente et récupération) 2003-2004 : Programme de prévention de la transmission du VIH et des hépatites chez les utilisateurs de drogues par injection, p. 7.

32. Denis BLAIS, hôpital Sainte-Justine, communication privée. 
entre l'Association des chirurgiens dentistes du Québec et le ministère de l'Environnement en 1992.

\subsection{Les acupuncteurs}

L'Ordre des acupuncteurs, qui existe depuis le $1^{\text {er }}$ juillet 1995 , compte entre 600 et 700 membres. Tous les acupuncteurs sont tenus de faire partie de l'Ordre.

Depuis le $1^{\mathrm{er}}$ avril 2003, l'Ordre exige l'utilisation d'aiguilles à usage unique. Pour se conformer au Règlement sur les déchets biomédicaux, l'Ordre exige que les acupuncteurs suivent la procédure suivante :

- déposer les DBM dans un contenant rigide résistant à la perforation, spécialement conçu à cette fin ;

- apposer une étiquette indiquant qu'il s'agit de matériel contaminé ;

- n'utiliser aucun contenant de verre ;

- conserver le contenant rempli scellé dans un lieu sûr, en attente de la prochaine collecte ;

- recourir à une compagnie spécialisée accréditée pour la collecte, le transport et l'élimination des DBM (certains $\mathrm{CH}$ munis d'un autoclave nous ont toutefois indiqué qu'ils traitaient et éliminaient aussi des DBM produits par des acupuncteurs);

- conserver le bon de collecte et le joindre au rapport annuel ;

- produire un rapport annuel, le conserver durant trois ans et le fournir sur demande ${ }^{33}$.

Les acupuncteurs ne réfrigèrent pas les contenants de DBM.

Selon le syndic de l'Ordre, "c'est la tolérance zéro quant aux dérogations à l'utilisation des aiguilles à usage unique et aux mesures à prendre pour l'élimination des aiguilles usagées ».

\subsection{Les pharmaciens}

Certains pharmaciens offrent des services, tels que tests de glycémie ou de cholestérol, qui requièrent l'utilisation d'instruments piquants ou tranchants. Les pharmaciens interrogés à ce sujet éliminent les DBM résultant de ces activités selon les normes : contenants réglementaires, décontamination dans un hôpital ou par Med-Tech avant l'enfouissement sanitaire.

Le code de déontologie des pharmaciens indique que « Le pharmacien doit, lorsque la demande lui en est faite par un de ses patients, collaborer à la récupération sécuritaire des médicaments périmés ou inutilisés, pour fins de destruction ${ }^{34} »$. Il n'existe pas de telle obligation relativement aux seringues et aux aiguille usagées. Toutefois, l'Ordre des pharmaciens du Québec « encourage les pharmaciens à mettre sur pied des programmes de récupération du matériel d'injection contaminés ${ }^{35} »$.

33. ORDRE DES ACUPUNCTEURS DU QUÉBEC, Guide de prévention des infections dans l'exercice de l'acupuncture, Montréal, Ordre des acupuncteurs du Québec, février 2000, p. 16.

34. Article 3.01.07.

35. ORDRE DES PHARMACIENS DU QUÉBEC, Informations professionnelles, $\mathrm{n}^{\circ}$ 94, avril 1998, p. 3. 
Dans les faits, les pratiques des pharmaciens en matière de récupération des seringues et des aiguilles utilisées par les individus à des fins domestiques varient considérablement d'un milieu à l'autre. La formule permettant l'accès le plus large à un moyen d'élimination sécuritaire consiste à offrir une installation où quiconque (diabétique, UDI ou autre, client ou pas) peut déposer luimême ses seringues et ses aiguilles usagées dans un contenant réglementaire, ce qui évite tout transvidage par le personnel de la pharmacie.

\subsubsection{Les seringues et les aiguilles provenant d'un usage thérapeutique}

En matière de récupération des seringues et des aiguilles utilisées à des fins thérapeutiques les pratiques suivantes ont été observées :

- le pharmacien donne des contenants réglementaires à ses clients et les récupère sans frais lorsqu'ils sont remplis ;

- il vend le premier contenant et remplace gratuitement les contenants remplis par la suite ;

- il donne des contenants de pilules vides aux clients et récupère sans frais les seringues et les aiguilles déposées dans ces contenants ;

- il vend tous les contenants ;

- il exige que le client achète ses contenants pour DBM pour accepter de récupérer les seringues et les aiguilles usagées ;

- il ne fournit aucun contenant ;

- il récupère les seringues et les aiguilles usagées de ses clients seulement ;

- il offre le service, mais sans le publiciser ;

- il ne fait aucune récupération.

\subsubsection{Les seringues provenant des programmes d'échange de seringues (PES)}

En 2003-2004, 409 pharmacies sur 1600 à travers le Québec étaient inscrites à la Liste officielle des centres d'accès aux seringues du Québec ${ }^{36}$. En général, les pharmacies qui vendent ou donnent des seringues aux UDI acceptent de les récupérer, comme l'indique le tableau 4.

On note toutefois des particularités dans certaines régions :

- Mauricie et Centre-du-Québec : aucune pharmacie n'est inscrite à la liste officielle des centres d'accès aux seringues ;

- Laurentides, Nord-du-Québec, Chaudière-Appalaches et Saguenay-Lac-Saint-Jean : toutes les pharmacies qui distribuent des seringues les récupèrent, mais ces pharmacies sont en très petit nombre ;

- Montérégie, Québec et Estrie: quelques rares pharmacies distribuent des seringues sans les récupérer ;

- Montréal : sur 150 pharmacies $^{37}$ qui distribuent des seringues aux UDI (en fait, toutes les vendent), seulement 44 les récupèrent.

36. Dans certains cas, il s'agit de plusieurs points de service d'une même pharmacie. 
Tableau 4

Les pharmacies qui distribuent des seringues aux UDI et celles qui les récupèrent, en 2003-2004

\begin{tabular}{lccc}
\hline Région & $\begin{array}{l}\text { Pharmacies qui } \\
\text { distribuent des seringues }\end{array}$ & $\begin{array}{l}\text { Pharmacies qui les } \\
\text { récupèrent }\end{array}$ & $\%$ \\
\hline $01-$ Bas-Saint-Laurent & 18 & 18 & 100 \\
\hline $02-$ Saguenay-Lac-Saint-Jean & 7 & 7 & 100 \\
\hline $03-$ Québec & 26 & 23 & 86 \\
\hline $04-$ Mauricie et Centre-du-Québec & 0 & 0 & - \\
\hline $05-$ Estrie & 40 & 38 & 95 \\
\hline $06-$ Montréal & 150 & 44 & 29 \\
\hline $07-$ Outaouais & 16 & 16 & 100 \\
\hline $08-$ Abitibi-Témiscamingue & 17 & 17 & 100 \\
\hline $09-$ Côte-Nord & 12 & 1 & 100 \\
\hline $10-$ Nord-du-Québec & 1 & 22 & 100 \\
\hline $11-$ Gaspésie-Îes-de-la-Madeleine & 22 & 4 & 100 \\
\hline $12-$ Chaudière-Appalaches & 4 & 13 & 100 \\
\hline $13-$ Laval & 13 & 29 & 100 \\
\hline $14-$ Lanaudière & 29 & 1 & 100 \\
\hline $15-$ Laurentides & 1 & 33 & 100 \\
\hline $16-$ Montérégie & 34 & & 97 \\
\hline
\end{tabular}

\subsection{Les tatoueurs et les perceurs}

Les artisans des trois salons de tatouage et perçage que nous avons visités se montrent soucieux de protéger leur clientèle contre les infections transmissibles par le sang. Dans chacun des cas, on utilise des aiguilles à usage unique, stérilisées et emballées individuellement. Après usage, les aiguilles sont cassées et déposées dans un collecteur pour DBM piquants et tranchants. Les instruments réutilisables qui peuvent avoir été en contact avec du sang sont stérilisés.

Cet échantillon n'est pas nécessairement représentatif des pratiques qui existent dans tous les milieux où l'on exerce les métiers de tatoueur et de perceur. Il n'existe aucun regroupement de tatoueurs et perceurs susceptible de formuler des recommandations ou des directives quant aux façons de procéder. Certaines personnes rencontrées ont dit souhaiter que le ministère de la Santé et des Services sociaux exerce une vigilance à cet égard et qu'il émette des normes de pratique

37. En 1998, l'Île de Montréal comptait 416 pharmacies communautaires. Source : DIRECTION DE LA SANTÉ PUBLIQUE DE MONTRÉAL-CENTRE, Services offerts aux UDI dans les pharmacies de Montréal et participation des pharmaciens au projet Stop Sida / UDI-Pharmacies, septembre 1996 juin 1998, Montréal, Régie régionale de la santé et des services sociaux de Montréal-Centre, septembre 2000, p. 18. 
sécuritaire, non seulement pour la prévention des infections, mais également en matière de prévention des traumatismes pouvant laisser des séquelles permanentes ${ }^{38}$.

Pour ce qui est des moyens de se débarrasser des contenants remplis de DBM piquants et tranchants, chacun se débrouille pour trouver une solution. La personne responsable d'un salon de tatouage et perçage apporte les contenants à un hôpital muni d'un autoclave. Une autre les apporte à un CLSC qui récupère et remplace les contenants réglementaires gratuitement. La troisième achète les contenants chez un distributeur d'équipement médical. Après avoir apporté quelques contenants de DBM à un CLSC qui se montrait peu empressé à les recevoir, elle les apporte maintenant à un organisme communautaire engagé dans la récupération de seringues auprès des UDI. Cet organisme les accepte sans réticence.

\subsection{Les électrolystes}

L'Association des électrolystes du Québec représente environ 500 personnes sur les 3000 qui exercent ce métier au Québec. Il ne s'agit pas d'un ordre professionnel. L'adhésion à l'organisme est volontaire.

Les nouveaux membres reçoivent une trousse d'accueil incluant des recommandations concernant la désinfection des instruments. La présidente de l'Association signale que la formation reçue en ce domaine varie selon les établissements d'enseignement.

Les électrolystes sont préoccupées par la prévention des infections. L'Association organise des conférences sur le sujet, ce qui suscite beaucoup d'intérêt. Au moment de notre rencontre, l'Association avait de la documentation sur la transmission du VIH et du VHB, mais ne disposait d'aucune information sur le VHC. Elle souhaiterait d'ailleurs recevoir des mises à jour sur ces questions de la part du MSSS.

L'Association recommande l'utilisation de filaments jetables qui ne serviront qu'une seule fois. Et, comment se débarrasse-t-on de ces filaments après usage ? Les électrolystes que nous avons rencontrées récupèrent les filaments soit dans un petit contenant pour DBM piquants et tranchants (0,5 litre), soit dans un flacon de pilules vides. Pour éliminer les contenants remplis de filaments (un par année, parfois moins), elles ont indiqué qu'il était préférable d'avoir un ami pharmacien qui accepte de les récupérer. L'Association souhaiterait d'ailleurs que les pharmaciens soient incités à récupérer les contenants de filaments usagés ou que l'on trouve un autre type d'endroit qui les accepterait.

\subsection{Les thanatologues}

Même si la Corporation des thanatologues du Québec n'est pas un ordre professionnel, elle s'est donné des règlements généraux, un comité de discipline et un code d'éthique. Une forte proportion des thanatologues sont membres de cet organisme.

38. Le ministère de la Santé et des services sociaux a publié, en 1999, un guide intitulé Tatoueurs et perceurs, protégez vos clients et protégez-vous contre le VIH et les hépatites B et C. Santé Canada a produit la même année un document intitulé Guide de prévention des infections: Pratiques de prévention des infections dans les services personnels: tatouage, perçage des oreilles, perçage corporel et électrolyse. Ces textes ne traitent que de la prévention des infections, et non pas des traumatismes pouvant laisser des séquelles permanentes. 
Les thanatopracteurs sont très préoccupés par les questions de santé et de prévention, affirme la secrétaire générale de la Corporation. On souhaite recevoir de l'information sur les moyens appropriés pour éviter la transmission de maladies et pour se débarrasser des DBM de façon sécuritaire. Le code d'éthique indique d'ailleurs que « le membre s'engage envers le public à [...] maintenir des normes hygiéniques et sanitaires élevées ».

Parmi les six thanatologues consultés, deux acheminent leurs DBM à un hôpital muni d'un autoclave. L'un d'eux y reçoit les services de décontamination et d'élimination des DBM gratuitement ; 1'autre doit verser $200 \$$ par année. Les quatre autres confient leurs DBM à Med-Tech.

\subsection{Les vétérinaires}

Pour connaître les pratiques et les préoccupations des vétérinaires en matière de récupération et d'élimination des DBM, notre consultation s'est limitée à l'Ordre des médecins vétérinaires. Cet ordre professionnel regroupe l'ensemble des quelque 2000 médecins vétérinaires du Québec.

Tout médecin vétérinaire doit se conformer à des normes minimales d'exercice. L'élimination sécuritaire des DBM fait partie des préoccupations. La vérification du mode d'élimination des DBM est au nombre des questions de routine qui sont posées au moment des inspections. On exige l'utilisation de contenants réglementaires. On indique que les vétérinaires doivent se défaire de leurs DBM selon les règlements en vigueur. Cependant, ce n'est pas l'Ordre qui voit à l'application du Règlement sur les déchets biomédicaux, mais le ministère de l'Environnement.

Plusieurs vétérinaires confient leurs DBM à Med-Tech. Certains hôpitaux munis d'autoclaves traitent aussi des DBM non anatomiques que des vétérinaires leur acheminent.

\subsection{Les autres utilisateurs professionnels}

Les autres utilisateurs de seringues et d'aiguilles à des fins professionnelles que nous avons consultés soit l'UQAM (centre de recherche), cégep du Vieux-Montréal (enseignement en soins infirmiers), Corporation Urgences Santé et Héma-Québec (qui produit de grandes quantités de DBM) utilisent tous les contenants réglementaires et acheminent leurs DBM à Med-Tech.

Certains hôpitaux munis d'autoclaves nous ont également indiqué qu'ils traitaient et éliminaient des DBM provenant de centres de recherche, de laboratoires, d'établissements d'enseignement, de résidences privées pour personnes âgées, de services ambulanciers et de services de santé de grandes entreprises.

\subsection{Les utilisateurs de seringues et d'aiguilles pour les autosoins}

Les diabétiques sont ceux qui utilisent le plus grand nombre de seringues pour les autosoins. Ils utilisent aussi d'autres instruments piquants et tranchants : les aiguilles pour stylo injecteur et les lancettes. D'autres maladies sont également traitées à domicile avec des seringues, notamment la sclérose en plaques. Nous n'avons pas mené de consultation auprès des individus, mais nous avons obtenu certains renseignements de la part des associations de malades. 


\subsubsection{Les diabétiques}

L'association Diabète Québec constate des lacunes dans les consignes qui sont données aux diabétiques. Ceux qui désirent se débarrasser de leurs seringues et de leurs aiguilles usagées de façon sécuritaire éprouvent souvent des difficultés. Telle pharmacie refuse tout simplement de les récupérer; telle autre les refuse si elles ne sont pas dans les contenants fournis par cette pharmacie. Certains CLSC les refusent aussi. Plusieurs diabétiques n'ont finalement d'autre possibilité que de les jeter aux ordures ménagères.

Une intervenante de l'association rappelle que non seulement les seringues, mais également les lancettes et les aiguilles des stylos injecteurs, sont susceptibles de causer des accidents et de transmettre des infections. "Les diabétiques, comme toute autre personne, peuvent être atteints du VIH, du VHC ou du VHB », dit-elle. Des intervenants à une clinique du diabète sont du même avis : « Le diabète de type 1 (10\% des cas) se déclare généralement durant le jeune âge. Chez les jeunes, on retrouve parfois des UDI qui peuvent avoir contracté une de ces infections. »

Dans un article publié au printemps 1998 dans Plein soleil ${ }^{39}$, le magazine de Diabète Québec, on formulait des recommandations concernant l'élimination des seringues, des aiguilles et des lancettes.

- Façon la plus efficace : les déposer dans un contenant de plastique épais et les rapporter à la pharmacie.

- Autre solution : les placer dans un contenant de plastique épais opaque avec bouchon ou couvercle pouvant être refermé solidement (p. ex. contenant d'eau de Javel ou d'assouplisseur) ou dans un contenant de métal (p. ex. pot de café), sceller le contenant avec un ruban adhésif avant de le jeter aux ordures.

- Ne pas remettre le capuchon sur la seringue ni briser l'aiguille afin d'éviter de se blesser. Si la personne souffre d'une baisse de l'acuité visuelle, le danger est encore plus grand.

- Ne pas couper l'aiguille avec un ciseau ou un instrument tranchant. Cette mesure est dangereuse car l'aiguille peut être propulsée ou échappée.

- Ne pas utiliser les contenants de verre.

- Ne pas retourner les contenants de DBM au recyclage.

- Si l'on utilise le coupe-aiguilles - ce qui rend la seringue inutilisable - traiter le reste de la seringue comme si l'aiguille n'avait pas été sectionnée car elle demeure piquante et le risque de contamination est toujours présent. Idéalement, retourner le coupe-aiguilles rempli à la pharmacie.

Il est impossible de savoir combien de diabétiques suivent ces consignes. Chose certaine, la préoccupation de l'élimination sécuritaire des seringues et des aiguilles est présente chez les diabétiques eux-mêmes, comme chez les professionnels de la santé qui les traitent.

39. Guylaine FORGET, «Les seringues, aiguilles et lancettes utilisées... Savez-vous comment en disposer? ", Arc-en-ciel, Association du diabète Laval-Laurentides M.R.C. Des Moulins inc., cité dans Plein soleil, Diabète Québec, printemps 1998, p. 13-14. 


\subsubsection{Les personnes atteintes de sclérose en plaques}

La Société de la sclérose en plaques reçoit, de la part de personnes atteintes de cette maladie et de la part de ses sections locales, des demandes d'information sur la façon de se débarrasser correctement des seringues usagées. Il n'y a pas de consigne claire à ce sujet.

Par l'intermédiaire d'une clinique spécialisée, nous avons appris que les fabricants de médicaments injectables pour le traitement de la sclérose en plaques fournissaient gratuitement les contenants de récupération aux patients. Il faut préciser que, contrairement à l'insuline, qui est un médicament peu coûteux, les immunomodulateurs utilisés par les personnes atteintes de sclérose en plaques sont très coûteux (de l'ordre de $14000 \$$ à $21000 \$$ par année). Les intervenants de cette clinique recommandent aux patients de rapporter les contenants remplis de seringues usagées à la pharmacie. "Depuis quelques années, il n’y a pas de problème, nous diton. Les patients coopèrent bien. »

\subsection{Les usagers de drogues par injection}

En 2002-2003, au Québec, 816898 seringues ont été récupérées, soit 73,8\% des seringues distribuées dans les centres d'accès aux seringues (CAS). Cette proportion est passée de $79 \%$ à $74 \%$ entre 2001-2002 et 2002-2003. Le taux de récupération a été de 74,9\% dans les centres spécialisés de prévention (p. ex. CACTUS Montréal) et de 68,9\% dans les autres CAS. Parmi ces autres CAS, ce sont les organismes communautaires à vocation multiple (p. ex. L'Anonyme) qui enregistrent généralement les meilleurs taux de récupération $(82,4 \%$ comparativement à $44,9 \%$ pour les CAS du réseau de la santé et les pharmacies) ${ }^{40}$.

Pour connaître les pratiques de récupération des seringues chez les UDI, nous nous sommes adressés à des organismes communautaires et à quelques intervenants du réseau public (CLSC, $\mathrm{CH}$, DSP) qui oeuvrent auprès d'eux dans différentes villes du Québec. Même si tous déploient des efforts immenses pour inciter les UDI à rapporter leurs seringues usagées ${ }^{41}$, un certain nombre de ces seringues se retrouvent dans l'environnement, notamment dans des parcs, sur la voie publique, dans les puisards, etc.

Les intervenants auprès des UDI que nous avons rencontrés avancent diverses explications aux lacunes dans la récupération des seringues usagées.

- Ceux qui jettent leurs seringues dans l'environnement seraient surtout des UDI qui ne demandent qu'un petit nombre de seringues aux organismes communautaires ou encore qui ne fréquentent pas ces organismes et se procurent des seringues ailleurs (p. ex. à la pharmacie).

- Certains pharmaciens refusent de récupérer les seringues usagées. Un organisme communautaire reçoit des seringues apportées par des UDI qui les achètent à la pharmacie mais que les pharmaciens refusent de récupérer. "Ces gens viennent porter leurs seringues souillées et ne demandent aucun matériel d'injection. »

40. Lina NOËL, Richard CLOUTIER et Marika MUNGER. Statistiques sur les services relatifs aux programmes de prévention du VIH et des hépatites B et $C$ offerts aux utilisateurs de drogues par injection du Québec, avril 2002 à mars 2003, ministère de la santé et des Services sociaux et Institut national de santé publique du Québec, mars 2004, p. 9.

41. À cette fin, l'Association des intervenants en toxicomanie du Québec a produit le magazine $F X$. Le MSSS a aussi produit une affiche et un autocollant dont les message est « Je rapporte mes seringues. » 
- Diverses mesures telles que la fermeture de piqueries et l'installation de caméras de surveillance contribuent à déplacer et à étaler le problème. Les travailleurs de rue, qui doivent parcourir des distances de plus en plus longues, sont dans l'impossibilité de revenir plusieurs fois par jour au site fixe d'échange de seringues; ils ne peuvent alors transporter tout le matériel stérile nécessaire et rapporter tous les contenants de seringues usagées que les UDI pourraient leur remettre ${ }^{42}$.

- Des UDI, craignant d'être interceptés par la police alors qu'ils sont en possession de seringues, même si cela n'a rien d'illégal, se débarrassent de leurs seringues usagées le plus rapidement possible, n'importe où.

- Il est difficile de trouver des moyens adéquats de sensibilisation : les UDI lisent peu, d'où l'efficacité limitée de l'information écrite.

- Certains UDI sont désorganisés au point où ils n'ont plus peur ni du sida, ni de la mort. Il est difficile alors de les convaincre de l'importance de s'occuper de la santé et de la sécurité des autres.

- Le nombre de collecteurs extérieurs de seringues est insuffisant : plus il y aura d'endroits où les UDI pourront déposer leurs seringues usagées, plus le taux de récupération sera élevé.

Outre l'échange de seringues dans les sites fixes, plusieurs initiatives sont prises par les organismes communautaires, en collaboration notamment avec les directions de santé publique, certains CLSC et certaines municipalités, pour améliorer le taux de récupération des seringues chez les UDI.

- À Montréal, on a créé des unités mobiles d'intervention qui se déplacent dans différents secteurs selon un horaire hebdomadaire préétabli.

- À Québec, un intervenant d'un organisme communautaire se rend aux divers endroits publics fréquentés par les UDI pour les sensibiliser à l'importance de récupérer les seringues. L'organisme établit des ententes avec des citoyens ou des commerçants à qui il donne une brève formation et fournit des contenants sécuritaires ainsi que des pinces. Sur appel de résidants, on se rend aussi sur place pour récupérer des seringues laissées à des endroits inappropriés.

- Dans certaines régions, on fournit gratuitement aux UDI des contenants sécuritaires adaptés à leurs besoins.

- Dans différentes villes, les travailleurs et travailleuses de rue transportent des contenants (pas toujours sécuritaires) dans leur sac à dos afin de récupérer des seringues qui, probablement, ne seraient pas rapportées au site fixe.

- Des organismes communautaires, des CLSC et des municipalités, entre autres, installent des collecteurs extérieurs de seringues (voir annexe II). À Montréal, actuellement, on compte 25 de ces collecteurs. On en trouve aussi quelques-uns dans d'autres villes.

- On organise des corvées saisonnières de ramassage de seringues dans l'environnement.

- Dans le quartier Centre-Sud de Montréal, le balayeur de rue, un employé d'un organisme communautaire, financé par la Direction de santé publique, ratisse régulièrement certains

42. Des organismes communautaires de Québec et de Gatineau souhaiteraient disposer d'un véhicule qui permettrait de visiter différents quartiers comme le font les unités mobiles de Montréal. Le temps épargné en déplacement par les travailleurs de rue pourrait ainsi être consacré à l'intervention. On pourrait aussi distribuer plus de matériel stérile et récupérer plus de seringues usagées. 
endroits fréquentés par les UDI afin de récupérer les seringues abandonnées dans l'environnement. Il en ramasse environ 2000 par année.

- À Montréal, également, une coalition regroupant la Ville de Montréal, la Direction de santé publique, des CLSC et des organismes communautaires a été formée en 2001, pour remédier à la présence de seringues dans le voisinage et préserver la qualité de vie des quartiers touchés par le phénomène ${ }^{43}$.

43. VILLE DE MONTRÉAL, DIRECTION DE SANTÉ PUBLIQUE DE MONTRÉAL-CENTRE, CLSC ET ORGANISMES COMMUNAUTAIRES, Coalition pour remédier à la présence de seringues à la traîne dans le voisinage (communiqué de presse), Montréal, 30 mai 2001. 


\section{Divers modes de collaboration déjà expérimentés}

L'objectif de récupérer et d'éliminer de façon sécuritaire toutes les seringues et toutes les aiguilles usagées provenant des diverses sources ne pourra se réaliser sans la mise en place d'un système intégré, c'est-à-dire d'une organisation permanente selon laquelle les opérations reliées à chacune des étapes de gestion des seringues et des aiguilles seront cohérentes. Cela exige la collaboration de plusieurs partenaires.

Diverses formes de collaboration, qui constituent des embryons plus ou moins développés d'un tel système intégré, existent dans plusieurs régions, principalement pour la récupération des seringues auprès des UDI. Mais il reste beaucoup à faire pour que tous les utilisateurs de seringues et d'aiguilles puissent vraiment se débarrasser de ces instruments, après usage, de façon sécuritaire à la fois pour eux-mêmes, pour les autres et pour l'environnement.

Des expériences de collaboration déjà réalisées dans certaines régions peuvent inspirer ceux qui souhaitent mettre en place un système intégré de gestion des seringues et des aiguilles usagées dans leur milieu. Nous en présentons quelques-unes, en étant persuadés qu'elles sont loin d'être les seules dignes de mention.

\subsection{Les centres d'accès aux seringues pour les UDI}

Les programmes d'échange de seringues (PES), instaurés en 1989 par les directions de santé publique, dans une perspective de réduction des méfaits liés à l'usage des drogues, permettent aux UDI de se procurer soit gratuitement, soit à prix modique, du matériel d'injection stérile. Les PES font appel à la collaboration de plusieurs acteurs. En 2003-2004, on comptait au Québec 700 centres d'accès aux seringues ainsi répartis :

- $53 \mathrm{CH}$,

- 187 CLSC,

- 3 cliniques médicales,

- 4 centres spécialisés,

- 44 organismes communautaires,

- 409 pharmacies ${ }^{44}$.

Ces services sont inégalement développés selon les régions et ils mériteraient sans doute d'être consolidés. Ainsi, il parait surprenant qu'aucun établissement public (CH ou CLSC) de la région de Québec ne soit inscrit à la liste des centres d'accès aux seringues. Étonnant aussi que, dans la région de la Mauricie et du Centre-du-Québec, les pharmacies soient totalement absentes du programme. À Montréal, c'est la faible proportion (29\%) des pharmacies qui acceptent de récupérer les seringues usagées, parmi celles qui vendent du matériel d'injection aux UDI, qui est préoccupante.

44. MINISTÈRE DE LA SANTÉ ET DES SERVICES SOCIAUX et INSTITUT NATIONAL DE SANTÉ PUBLIQUE DU QUÉBEC. Liste officielle des centres d'accès aux seringues du Québec (distribution, vente et récupération) 2003-2004: Programme de prévention de la transmission du VIH et des hépatites chez les utilisateurs de drogues par injection. p. 7. 
Par ailleurs, plusieurs $\mathrm{CH}$, surtout parmi ceux qui sont munis d'autoclaves, acceptent de traiter et d'éliminer sans frais les seringues récupérées auprès des UDI. Plusieurs autres se montrent disposés à offrir ce service si la demande leur en est faite.

\subsection{Les services offerts par les $\mathrm{CH}$ munis d'autoclaves}

La majorité des $\mathrm{CH}$ munis d'autoclaves offrent le service de décontamination et d'élimination des DBM aux autres missions du même établissement, aux autres établissements publics de santé et à des organismes communautaires du milieu. Plusieurs offrent aussi ce service, à prix modique, à d'autres « clients » tels que les cliniques médicales, les cliniques dentaires, les acupuncteurs, les thanatologues, les vétérinaires, etc.

En voici quelques exemples :

- le $\mathrm{CH}$ Pierre-Boucher, à Longueuil, décontamine et élimine les DBM de 31 clients, soit 2 CLSC, 6 CHSLD, 14 cliniques médicales, 5 cliniques dentaires, 2 laboratoires, 1 acupuncteur et 1 service ambulancier;

- le Centre de santé et de services sociaux de la Haute-Yamaska (Granby) compte 42 clients pour ce service, soit 1 CLSC, 3 CHSLD, 9 cliniques médicales, 14 cliniques dentaires, 2 établissements de formation, 1 usine, 5 cliniques vétérinaires, 6 maisons funéraires, 1 jardin zoologique ;

- l'Hôtel-Dieu de Saint-Jérôme offre le même service à 51 clients, soit 1 CLSC, 10 CHSLD, 2 centres de réadaptation, 8 dentistes, 23 cliniques médicales, 1 entreprise funéraire, 1 vétérinaire, 1 centre de recherche, 1 organisme communautaire, 1 communauté religieuse, 1 infirmière et 1 centre de détention;

- les $\mathrm{CH}$ de la Côte-Nord affirment répondre à toutes les demandes et cela, sans frais ;

- le CH Saint-Eustache ne réclame aucuns frais à ceux qui apportent de petites quantités de DBM à traiter et à éliminer, soit les organismes communautaires, les cliniques médicales privées, les services infirmiers privés à domicile, les dentistes et les acupuncteurs.

En rendant accessible gratuitement ou à peu de frais leurs ressources humaines et matérielles pour le traitement et l'élimination des DBM, les $\mathrm{CH}$ diminuent d'autant les motifs que pourraient avoir les utilisateurs de seringues et d'aiguilles de se débarrasser de ces instruments de façon inappropriée. Dans la plupart des cas, toutefois, le service est offert surtout aux professionnels qui font usage de seringues et d'aiguilles. D'autres utilisateurs tels que les tatoueurs, les perceurs et les électrolystes, de même que ceux qui se servent de seringues et d'aiguilles pour les autosoins, éprouvent souvent de la difficulté à trouver un endroit où ils pourront éliminer leurs DBM piquants et tranchants de façon sécuritaire.

\subsection{La mobilisation de différents milieux pour la récupération des seringues souillées dans la région de la Mauricie et du Centre-du-Québec}

En 1998, la Direction de santé publique de la Mauricie et du Centre-du-Québec a mobilisé des intervenants de différents milieux tels que CLSC, équipes de santé et sécurité du travail, municipalités et travailleurs de rue afin d'élaborer une stratégie visant à prévenir les incidents reliés aux seringues souillées.

Plusieurs actions ont été réalisées, dont la production et la diffusion d'une affiche à l'intention de la population, la production et la diffusion d'un dépliant à l'intention des travailleurs municipaux, 
la formation des animateurs de terrains de jeu et l'implantation d'une politique régionale sur la récupération des seringues souillées.

Cette politique régionale, toujours en vigueur, précise le rôle des différents intervenants et organismes concernés. Par exemple :

- les organismes communautaires associés aux PES obtiennent gratuitement de la Direction de santé publique les contenants réglementaires, qu'ils peuvent apporter à un centre hospitalier lorsqu'ils sont remplis. La DSP assume les frais de décontamination et d'élimination de ces DBM ;

- les CLSC acceptent de récupérer les seringues des UDI et celles qui sont ramassées dans l'environnement par des individus ;

- Info-Santé CLSC donne de l'information aux personnes qui trouvent des seringues sur la façon sécuritaire de les manipuler et sur le moyen de s'en débarrasser.

La politique comprend aussi un mécanisme de signalement des incidents ou de la présence de seringues (appel d'Info-santé à la DSP) ainsi qu'une procédure d'intervention à la suite d'un signalement (appel de la DSP au secteur des travaux publics des municipalités ou aux travailleurs de rue afin qu'ils se rendent sur place pour récupérer ces seringues). Le tout permet à la DSP de tracer un portrait de la réalité et de mieux cibler ses interventions de prévention.

\subsection{Le comité de concertation pour la réduction des seringues à la traîne dans l’environnement à Montréal}

Deux ans après son lancement officiel, la coalition regroupant la Ville de Montréal, la direction de santé publique, des CLSC et des organismes communautaires, formée pour remédier à la présence de seringues dans l'environnement, estimait que la situation avait évolué positivement. Plusieurs mesures et procédures ont été mises en place de manière à réduire le nombre de seringues dans l'environnement et le risque qu'elles représentent pour la population, notamment :

- l'identification d'un répondant unique (Service des travaux publics de la ville de Montréal) accessible 24 heures sur 24 pour le ramassage rapide des seringues signalées sur la voie publique ;

- l'augmentation substantielle du nombre de parcs faisant l'objet de corvées systématiques de récupération par le Service des parcs ;

- l'installation d'un nombre croissant de collecteurs extérieurs sécuritaires et fonctionnels dans les zones considérées plus problématiques ;

- l'intensification des messages d'élimination sécuritaire auprès des usagers des PES ;

- la distribution de contenants de récupération sécuritaires pour les UDI ;

- la multiplication des messages préventifs à la population et à certains groupes à risque ;

- un meilleur monitorage des seringues distribuées et récupérées ;

- l'intégration dans les procédures d'Info-santé des mesures à prendre lorsque des seringues sont trouvées dans l'environnement ${ }^{45}$.

45. COMITÉ DE CONCERTATION POUR LA RÉDUCTION DES SERINGUES À LA TRAÎNE DANS L'ENVIRONNEMENT À MONTRÉAL, État de situation à l'hiver 2003, 3 avril 2003, p.1. 
L'action de cette coalition est centrée essentiellement sur les seringues trouvées dans l'environnement, phénomène que l'on associe à l'usage de drogues par injection. La question des seringues et des aiguilles provenant d'autres usages (p. ex. les autosoins) et des piqûres accidentelles qui résultent de leur élimination inadéquate n'a pas été traitée par cette instance.

\subsection{La récupération des seringues usagées de diabétiques en Abitibi-Témiscamingue}

En plus du programme de récupération de seringues chez les UDI, la DSP de l'AbitibiTémiscamingue a décidé d'offrir aux autres usagers de seringues à domicile (surtout des diabétiques) les moyens de se débarrasser de leurs seringues usagées de façon sécuritaire ${ }^{46,47}$.

Jusqu'en 1999, la majorité des diabétiques jetaient leurs seringues aux ordures ménagères. On déplorait des piqûres accidentelles chez les enfants (entre 1 et 5 enfants par année) et chez plusieurs travailleurs, surtout ceux qui étaient affectés à la collecte des ordures ménagères et aux centres de tri. Les diabétiques semblaient intéressés à un service régional de récupération pourvu que cela soit gratuit. Les pharmaciens se sont dits prêts à collaborer à un projet régional pourvu que cela ne coûte rien et que cela ne complique pas leur tâche.

Après validation au moyen d'un projet pilote, une entente est intervenue entre la régie régionale de la santé et des services sociaux, les hôpitaux munis d'autoclaves, les pharmaciens et la direction régionale du ministère de l'Environnement sur le processus suivant :

- le pharmacien sensibilise le client diabétique à la nécessité d'éliminer ses seringues de façon sécuritaire (distribution d'un dépliant) ;

- on fait la promotion du coupe-aiguilles pour réduire le volume de DBM ;

- le diabétique est invité à rapporter ses seringues ou ses aiguilles usagées au pharmacien ;

- le pharmacien les récupère gratuitement et les apporte à l'hôpital ;

- l'hôpital procède, sans frais, à la décontamination de ces DBM et les achemine au site d'enfouissement sanitaire avec ses propres déchets.

La régie régionale a aussi pu compter sur l'appui des cliniques pour diabétiques et des associations locales de diabétiques.

Pour réduire les coûts, outre l'utilisation du coupe-aiguilles, la DSP approvisionne les hôpitaux en chaudières de plastique commerciales (fournies par McDonald's, Tim Hortons, etc.). Les hôpitaux les remettent aux pharmaciens pour la récupération des seringues.

Ceux qui ont réalisé cette expérience dans la région en ont dégagé quelques leçons, ce qui les a amenés à « corriger le tir ${ }^{48} »$.

- On a découvert que les diabétiques n'étaient pas les seuls «fautifs". Il y avait aussi les utilisateurs de la dialyse péritonéale. On a donc élaboré un message de sensibilisation à leur intention.

46. Daniel GAGNÉ, La récupération des seringues usagées de diabétiques en Abitibi-Témiscamingue (présentation PowerPoint), Direction de santé publique de l'Abitibi-Témiscamingue, mai 2004.

47. RÉGIE RÉGIONALE DE LA SANTÉ ET DES SERVICES SOCIAUX EN ABITIBITÉMISCAMINGUE. Projet visant à faciliter la récupération des seringues utilisées à domicile en Abitibi-Témiscamingue, 22 mai 1997, $11 \mathrm{p}$.

48. Daniel GAGNÉ, op. cit. 
- On a constaté une confusion dans les messages : afin de récupérer les seringues usagées, des gens utilisaient les bacs de récupération pour matières recyclables, ce qui a entraîné une augmentation des piqûres accidentelles chez les travailleurs des centres de tri. On a donc cessé d'utiliser le mot récupération dans les messages publics.

- Des autocollants invitant les usagers à rapporter leurs seringues usagées à leur pharmacien ont été apposés sur les boîtes de seringues neuves. De plus, un message a été diffusé à la télévision afin de sensibiliser la population aux risques que présentent les seringues jetées dans les ordures ménagères et dans les bacs de recyclage, et pour inviter les usagers à les rapporter à leur pharmacien (service gratuit).

- Dans certains cas, les chaudières commerciales présentent l'inconvénient de fondre au stérilisateur, ce qui peut endommager l'appareil ${ }^{49}$. Au moins un hôpital a décidé de ne plus les utiliser et de fournir aux pharmaciens de grands contenants pour DBM dans lesquels ils déposent les seringues et les aiguilles usagées apportées par les clients dans des contenants non sécuritaires.

- À défaut de pouvoir fournir des contenants à DBM à tous les usagers de seringues et d'aiguilles, au moins un pharmacien fournit au client un contenant à pilules vide (format 1000 comprimés) afin qu'il y dépose ses seringues, ce qui est, évidemment, plus sécuritaire que les sacs de plastique, les sacs de papier, les bouteilles d'eau, etc. dans lesquels les clients rapportent parfois leurs seringues.

49. C'est aussi le cas d'autres contenants en plastique qui ne sont pas prévus pour supporter le degré de chaleur que doit atteindre l'autoclave et cela, non seulement en Abitibi-Témiscamingue. 



\section{Des obstacles à surmonter}

Au cours de nos consultations, nous avons cherché systématiquement à savoir quels étaient les obstacles à la mise en place d'un système intégré de récupération des seringues et des aiguilles usagées. L'analyse des réponses reçues à nos questionnaires écrits et des témoignages recueillis lors de nos rencontres nous a permis de cerner ce qui nous semble constituer les huit principaux obstacles. À notre avis, tous ces obstacles sont surmontables. Dans la plupart des cas, les expériences des uns peuvent constituer des solutions aux problèmes vécus ou appréhendés par les autres.

\subsection{Un règlement qui ne s’applique pas aux individus}

La présence de seringues et d'aiguilles à des endroits inappropriés est souvent attribuée à la négligence de certaines personnes qui en font un usage soit professionnel, soit domestique. Sans nier que, dans ce domaine comme dans plusieurs autres, certaines personnes puissent avoir des comportements irresponsables, il nous semble qu'il faille chercher ailleurs les principales causes de ce phénomène.

Ainsi, nous avons appris à plusieurs de nos interlocuteurs, y compris à des représentants d'organismes d'envergure nationale et des représentants de municipalités, que le Règlement sur les déchets biomédicaux ne s'applique pas aux DBM provenant de l'usage domestique. Lorsque les individus jettent leurs seringues et leurs aiguilles aux ordures ménagères, ils ne contreviennent aucunement au Règlement.

Par ailleurs, nous avons entendu plusieurs témoignages selon lesquels

- des diabétiques, qui trouvent inapproprié de jeter les seringues, les aiguilles et les lancettes à la poubelle, en accumulent de grandes quantités à la maison, en attendant de trouver un endroit où ils pourraient s'en débarrasser de façon sécuritaire ;

- d'autres ont tenté en vain de les apporter à la pharmacie ou au CLSC, mais on refusait de les récupérer ;

- des producteurs de petites ou très petites quantités de DBM (p. ex. soins infirmiers à domicile, tatoueurs, électrolystes) sont accueillis sans enthousiasme lorsqu'ils apportent leurs DBM à la pharmacie ou au CLSC.

Ces obstacles pourraient être éliminés, du moins en grande partie, par des modifications au Règlement sur les déchets biomédicaux, par la désignation de lieux où les usagers pourraient apporter leurs contenants de seringues et d'aiguilles en ayant l'assurance qu'ils seront acceptés, de même que par une campagne d'information et de sensibilisation auprès des différents publics concernés.

\subsection{Des consignes contradictoires}

Les consignes données aux utilisateurs de seringues à des fins domestiques sont contradictoires, tant en ce qui concerne le traitement à donner aux aiguilles que les contenants à utiliser et les moyens de les éliminer. Dans une même région, voire dans une même ville, les consignes sont différentes. Pour ce qui est des contenants, la liste des options proposées - parfois selon un ordre de priorité, parfois comme un moindre mal - est impressionnante. Quant au moyen de s'en débarrasser, la contradiction typique est la suivante : le CLSC dit à l'usager de rapporter ses 
seringues et ses aiguilles à la pharmacie alors que le pharmacien considère que c'est le CLSC qui a la responsabilité de les récupérer. Cette absence de consigne claire empêche le service InfoSanté CLSC d'établir un protocole qui pourrait être diffusé à l'échelle du Québec.

Le groupe de travail a examiné les différentes consignes qui sont données aux usagers de seringues et d'aiguilles, du point de vue de leurs incidences sur la santé et la sécurité de l'utilisateur, sur la santé et la sécurité de la population, sur la santé et la sécurité des travailleurs et sur la préservation de la qualité de l'environnement. Le tableau 5 résume le point de vue du groupe de travail sur les différentes consignes données à travers le Québec. Si aucune formule ne présente que des avantages, certaines sont carrément à proscrire (p. ex. l'usage de contenants en verre).

Force est de constater que les pratiques les plus sécuritaires pour la population et pour les travailleurs qui font la manutention et le traitement des DBM (p. ex. l'utilisation de contenants réglementaires pour DBM) sont les plus coûteuses et celles qui ont pour effet d'acheminer les plus grandes quantités de matières plastiques aux sites d'enfouissement.

D'autres pratiques, telles que l'usage du coupe-aiguilles, ou encore celles qui consistent à couper ou à casser l'aiguille offrent l'avantage de rendre la seringue inutilisable. À l'instar de plusieurs intervenants qui se sont exprimés sur cette question, le groupe de travail ne saurait faire la recommandation d'utiliser le coupe-aiguilles étant donné les risques de blessures (et, éventuellement d'infection) qui persistent en raison du bout d'aiguille qui reste attaché à la seringue. La seringue elle-même devrait être considérée comme les autres DBM. Le groupe de travail ne saurait davantage recommander de casser ou de couper l'aiguille, étant donné le risque d'échapper l'aiguille et de ne pas la retrouver, risque d'autant plus important que certains utilisateurs (p. ex. plusieurs diabétiques) ont une acuité visuelle déficiente.

La consigne de ne pas replacer le capuchon sur l'aiguille - importante pour les utilisateurs professionnels de seringues - a pour effet de rendre les seringues usagées plus dangereuses pour la population et pour les travailleurs.

Les contenants domestiques en plastique considérés comme résistant à la perforation, tels que bouteilles d'eau de Javel ou d'assouplisseur, ne résistent pas toujours à la perforation. De plus, ces contenants, tout comme les boîtes en métal, sont opaques, ce qui risque davantage de causer des accidents chez ceux qui font la manutention des déchets (concierges, éboueurs, travailleurs des centres de tri), si ces contenants ne portent pas clairement la mention « Pas pour recyclage» et s'ils sont jetés parmi les ordures ménagères.

Le groupe de travail est d'avis qu'il faut privilégier les approches les plus sécuritaires pour la population et pour les travailleurs. Des solutions doivent être trouvées pour rendre accessibles à moindre coût les contenants réglementaires pour DBM piquants et tranchants et pour en favoriser un usage judicieux, notamment par le tri à la source des déchets qui y sont déposés.

Pour ce qui est de l'endroit où se débarrasser des contenants remplis de seringues ou d'aiguilles usagées, un très grand nombre d'intervenants - tant dans le réseau public de santé qu'à l'extérieur - ont manifesté leur malaise devant la formule qui consiste à les jeter aux ordures ménagères. En de nombreux endroits, on se heurte cependant à l'absence d'autre solution. 


\section{Tableau 5}

Avantages et inconvénients des diverses consignes données aux utilisateurs de seringues et d'aiguilles

\begin{tabular}{|c|c|c|c|c|}
\hline Consigne & $\begin{array}{l}\text { Pour protéger la santé et la } \\
\text { sécurité de l'utilisateur }\end{array}$ & $\begin{array}{l}\text { Pour protéger la santé et la } \\
\text { sécurité de la population }\end{array}$ & $\begin{array}{l}\text { Pour protéger la santé et la } \\
\text { sécurité des travailleurs }\end{array}$ & $\begin{array}{l}\text { Pour préserver la qualité } \\
\text { de l'environnement }\end{array}$ \\
\hline $\begin{array}{l}\text { 1. Couper l'aiguille avec un } \\
\text { coupe-aiguilles } \\
\text { (dans le but de réduire le } \\
\text { volume de DBM à } \\
\text { entreposer et à } \\
\text { décontaminer). }\end{array}$ & $\begin{array}{l}\text { Pas d'inconvénient si la personne } \\
\text { coupe les aiguilles qu'elle a } \\
\text { utilisées elle-même et si elle n'est } \\
\text { pas affectée par des troubles de } \\
\text { vision ni par des tremblements. }\end{array}$ & $\begin{array}{l}\text { La seringue ne peut être réutilisée. } \\
\text { Le bout d'aiguille qui reste } \\
\text { attaché à la seringue peut causer } \\
\text { des blessures et, éventuellement, } \\
\text { des infections. }\end{array}$ & $\begin{array}{l}\text { Le bout d'aiguille qui reste } \\
\text { attaché à la seringue peut causer } \\
\text { des blessures et, éventuellement, } \\
\text { des infections. }\end{array}$ & $\begin{array}{l}\text { Aucune différence : le tout } \\
\text { se retrouvera au site } \\
\text { d'enfouissement. }\end{array}$ \\
\hline $\begin{array}{l}\text { 2. Couper l'aiguille avec des } \\
\text { ciseaux } \\
\text { ou } \\
\text { casser l'aiguille et } \\
\text { l'insérer dans le piston. }\end{array}$ & $\begin{array}{l}\text { Risque d'échapper l'aiguille et de } \\
\text { ne pas la retrouver, surtout } \\
\text { lorsque l'acuité visuelle est } \\
\text { déficiente. }\end{array}$ & $\begin{array}{l}\text { La seringue ne peut être réutilisée. } \\
\text { L'entourage pourrait se blesser } \\
\text { sur une aiguille perdue et, } \\
\text { éventuellement contracter une } \\
\text { infection. }\end{array}$ & $\begin{array}{l}\text { Pas de problème si les aiguilles } \\
\text { sont déposées dans des contenants } \\
\text { sécuritaires. }\end{array}$ & $\begin{array}{l}\text { Aucune différence : le tout } \\
\text { se retrouvera au site } \\
\text { d'enfouissement. }\end{array}$ \\
\hline $\begin{array}{l}\text { 3. Replacer le capuchon sur } \\
\text { l'aiguille. }\end{array}$ & $\begin{array}{l}\text { Risque d'accident, mais pas } \\
\text { d'infection pour l'usager } \\
\text { domestique. } \\
\text { Risque d'infection pour } \\
\text { l'utilisateur professionnel. }\end{array}$ & $\begin{array}{l}\text { Avantage dans le cas de seringues } \\
\text { laissées à des endroits } \\
\text { inappropriés. }\end{array}$ & $\begin{array}{l}\text { Avantage pour ceux qui font la } \\
\text { manutention, le transport, la } \\
\text { décontamination et l'élimination. }\end{array}$ & $\begin{array}{l}\text { Aucune différence : le tout } \\
\text { se retrouvera au site } \\
\text { d'enfouissement. }\end{array}$ \\
\hline $\begin{array}{l}\text { 4. Ne jamais replacer le } \\
\text { capuchon sur l'aiguille. }\end{array}$ & $\begin{array}{l}\text { L'usager domestique peut se } \\
\text { blesser mais ne peut s'infecter } \\
\text { avec ses propres seringues. } \\
\text { Important pour l'utilisateur } \\
\text { professionnel. }\end{array}$ & $\begin{array}{l}\text { Plus de risques dans le cas de } \\
\text { seringues laissées à des endroits } \\
\text { inappropriés. }\end{array}$ & $\begin{array}{l}\text { Plus de risques pour ceux qui font } \\
\text { la manutention, le transport, la } \\
\text { décontamination et l'élimination. }\end{array}$ & $\begin{array}{l}\text { Aucune différence : le tout } \\
\text { se retrouvera au site } \\
\text { d'enfouissement. }\end{array}$ \\
\hline
\end{tabular}


Tableau 5 (suite)

Avantages et inconvénients des diverses consignes données aux utilisateurs de seringues et d'aiguilles

\begin{tabular}{|c|c|c|c|c|}
\hline Consigne & $\begin{array}{l}\text { Pour protéger la santé et la } \\
\text { sécurité de l'utilisateur }\end{array}$ & $\begin{array}{l}\text { Pour protéger la santé et la } \\
\text { sécurité de la population }\end{array}$ & $\begin{array}{l}\text { Pour protéger la santé et la } \\
\text { sécurité des travailleurs }\end{array}$ & $\begin{array}{l}\text { Pour préserver la qualité de } \\
\text { l'environnement }\end{array}$ \\
\hline $\begin{array}{l}\text { 5. Déposer les seringues et } \\
\text { les aiguilles dans un } \\
\text { contenant à DBM } \\
\text { piquants et tranchants } \\
\text { réglementaire. }\end{array}$ & $\begin{array}{l}\text { Pas d'effet pour l'usager } \\
\text { domestique. } \\
\text { Moyen le plus sécuritaire pour } \\
\text { l'utilisateur professionnel. }\end{array}$ & $\begin{array}{l}\text { Moyen le plus sécuritaire, dans la } \\
\text { mesure où les contenants sont } \\
\text { bien fermés et déposés à des } \\
\text { endroits appropriés. }\end{array}$ & $\begin{array}{l}\text { Moyen plus sécuritaire que les } \\
\text { contenants domestiques réutilisés } \\
\text { qui ne résistent pas toujours à la } \\
\text { perforation et ont déjà causé des } \\
\text { piqûres accidentelles. }\end{array}$ & $\begin{array}{l}\text { Beaucoup de matières } \\
\text { plastiques sont acheminées au } \\
\text { site d'enfouissement et y } \\
\text { demeureront durant de } \\
\text { nombreuses années. }\end{array}$ \\
\hline
\end{tabular}

6. Déposer les seringues et Pas d'inconvénient pour l'usager les aiguilles dans un contenant en plastique résistant à la perforation muni d'un couvercle (eau de Javel, assouplisseur, boissons gazeuses, beurre d'arachide, etc.)

Avantage : gratuit.
Pas de problème si ces contenants sont déposés à des endroits appropriés.
Risques pour ceux qui font la manutention, le transport, la décontamination et l'élimination.

Risques pour l'utilisateur professionnel.
Plusieurs de ces contenants ne résistent pas complètement à la perforation et ont déjà été la cause d'accidents.
7. Déposer les seringues et Pas d'inconvénient pour l'usager les aiguilles dans un contenant en métal (contenant de café avec couvercle en métal, boîte de conserve ouverte avec un ouvreboîte pointu et refermée avec du ruban adhésif).

Avantage : gratuit.

\section{domestique.}

Pas de risques pour l'utilisateur

professionnel à la condition qu'un

tel contenant soit fermé

solidement.
Pas de problème si ces contenants sont solidement fermés et son déposés à des endroits appropriés.

Pas de problème si les contenants sont solidement fermés, bien identifiés « Pas pour recyclage » et déposés à des endroits appropriés.
Réutilise des contenants qui, au mieux, seraient recyclés, mais dont plusieurs se retrouveraient au site d'enfouissement.

Doivent être déposés dans d'autres contenants sécuritaires pour être acceptés par MedTech et, en général, par les établissements qui font la décontamination par autoclave. 
Tableau 5 (suite)

Avantages et inconvénients des diverses consignes données aux utilisateurs de seringues et d'aiguilles

\begin{tabular}{|c|c|c|c|c|}
\hline Consigne & $\begin{array}{l}\text { Pour protéger la santé et la } \\
\text { sécurité de l'utilisateur }\end{array}$ & $\begin{array}{l}\text { Pour protéger la santé et la } \\
\text { sécurité de la population }\end{array}$ & $\begin{array}{l}\text { Pour protéger la santé et la } \\
\text { sécurité des travailleurs }\end{array}$ & $\begin{array}{l}\text { Pour préserver la qualité de } \\
\text { l'environnement }\end{array}$ \\
\hline $\begin{array}{l}\text { 8. Déposer les seringues et } \\
\text { les aiguilles dans un } \\
\text { contenant en verre. } \\
\text { Avantage : gratuit. }\end{array}$ & $\begin{array}{l}\text { Aucun inconvénient pour l'usager } \\
\text { domestique. } \\
\text { Risques pour l'utilisateur } \\
\text { professionnel si le contenant se } \\
\text { brise, p. ex. à l'occasion d'un } \\
\text { transport. }\end{array}$ & $\begin{array}{l}\text { Risques de blessures et, } \\
\text { éventuellement, d'infection si le } \\
\text { contenant se brise. }\end{array}$ & $\begin{array}{l}\text { Si le contenant se brise, risques } \\
\text { pour ceux qui font la } \\
\text { manutention, le transport, la } \\
\text { décontamination et l'élimination. }\end{array}$ & $\begin{array}{l}\text { Avantage de réutiliser des } \\
\text { contenants qui, au mieux. se } \\
\text { retrouveraient au recyclage, } \\
\text { mais dont plusieurs se } \\
\text { retrouveraient au site } \\
\text { d'enfouissement. }\end{array}$ \\
\hline $\begin{array}{l}\text { 9. Remplir le contenant } \\
\text { d'eau de Javel. }\end{array}$ & $\begin{array}{l}\text { Aucun inconvénient pour l'usager } \\
\text { domestique, sauf le coût de l'eau } \\
\text { de Javel et le risque de respirer } \\
\text { des vapeurs de chlore. }\end{array}$ & $\begin{array}{l}\text { Trop de conditions à respecter } \\
\text { pour obtenir l'assurance d'une } \\
\text { véritable décontamination. }\end{array}$ & $\begin{array}{l}\text { En augmentant le poids du } \\
\text { contenant, on augmente le risque } \\
\text { qu'il éclate ou que le couvercle se } \\
\text { détache. }\end{array}$ & Aucun avantage. \\
\hline $\begin{array}{l}\text { 10. Indiquer sur le contenant } \\
\text { « Pas pour le recyclage ». }\end{array}$ & $\begin{array}{l}\text { Aucun effet pour l'usager } \\
\text { domestique. }\end{array}$ & $\begin{array}{l}\text { Pas de problème si les contenants } \\
\text { sont déposés à des endroits } \\
\text { appropriés. }\end{array}$ & $\begin{array}{l}\text { Utile, surtout si le contenant n'est } \\
\text { pas transparent. } \\
\text { Réduit les risques de blessures } \\
\text { pour les travailleurs de centres de } \\
\text { tri. }\end{array}$ & Aucun effet. \\
\hline
\end{tabular}




\subsection{Le refus de certains pharmaciens de récupérer les seringues et les aiguilles usagées}

L'obstacle qui a été le plus souvent mentionné est le refus de certains pharmaciens de récupérer les seringues et les aiguilles usagées. Comme les seringues et les aiguilles utilisées pour les autosoins sont normalement vendues à la pharmacie, bon nombre d'intervenants trouvent qu'il serait logique que les clients rapportent leurs contenants de seringues usagées à la pharmacie au moment où ils s'y rendent pour en acheter de nouvelles.

De leur côté, des pharmaciens nous ont fait part de problèmes vécus en ce domaine :

- le pharmacien qui accepte de récupérer les seringues usagées écope de celles qui sont achetées chez des compétiteurs qui, eux, ne les récupèrent pas ;

- les clients rapportent leurs seringues à la pharmacie dans toute sorte de contenants, y compris des sacs de plastique et des sacs de papier, ce qui a déjà causé des accidents ;

- il y a pénurie de pharmaciens, la récupération des médicaments périmés représente déjà un surcroît de tâche pour lequel les pharmaciens ne sont pas rémunérés ;

- on manque d'espace pour l'entreposage des contenants de seringues usagées ;

- les coûts d'élimination des seringues usagées sont prohibitifs.

D'autres nous ont signalé des problèmes qu'ils appréhendaient, notamment la crainte de procédures compliquées et de tracasseries administratives s'ils apportaient à un hôpital muni d'un autoclave les seringues et les aiguilles usagées récupérées auprès des individus. Mais les principales appréhensions étaient liées à la récupération des seringues usagées des UDI :

- peur d'être identifié comme site d'échange de seringues et d'attirer ainsi une clientèle désagréable à l'égard du personnel ou des autres clients ;

- crainte de vol ou de vandalisme.

Le groupe de travail est d'avis que les problèmes de manque d'espace et de surcroît de travail pourraient être réduits sensiblement par une augmentation du nombre d'endroits où les individus peuvent apporter les seringues et les aiguilles usagées. Il estime cependant que ce service rendu à la société ne doit pas mettre en danger la santé et la sécurité des pharmaciens ni celle de leur personnel, d'où l'importance de faciliter l'accès aux contenants pour DBM piquants et tranchants. Des solutions doivent également être trouvées pour que les pharmaciens qui récupèrent les seringues et les aiguilles usagées n'aient pas à en supporter les coûts d'élimination. À cet égard, l'expérience de la région de l'Abitibi-Témiscamingue pourrait être appliquée dans d'autres régions.

En ce qui a trait aux problèmes appréhendés, précisons que les $\mathrm{CH}$ qui offrent le service de décontamination et d'élimination des DBM à des clients extérieurs ont mis au point une procédure simple de réception et de facturation. De tous les « clients » que nous avons interrogés, aucun ne s'est plaint ni de la qualité du service ni de tracasseries administratives.

Les craintes au sujet du vol, du vandalisme ou de diverses formes de violence liées au fait de vendre des seringues sans ordonnance aux UDI et de récupérer le matériel souillé ne sont pas fondées, du moins si l'on se fie aux résultats d'un projet pilote qui a été réalisé à Montréal du $1^{\text {er }}$ avril 1994 au 30 avril 1995. L'expérience de 8 pharmacies participantes (qui acceptaient de vendre des seringues aux UDI et récupéraient le matériel souillé) et de 8 pharmacies témoins qui 
vendaient des seringues, mais pas nécessairement aux UDI, a montré que les incidents rapportés dans les pharmacies participantes n'étaient pas plus nombreux que ceux qui s'étaient produits dans les pharmacies témoins ${ }^{50}$.

Une autre étude réalisée auprès des pharmacies de Montréal qui «vendaient des seringues à des personnes non diabétiques sans prescription » (Projet Stop Sida/UDI-Pharmacies) a révélé que le nombre d'incidents était relativement peu élevé. Ces pharmacies, dont l'expérience totalisait environ 437 années de vente de seringues, ont rapporté 36 cas de difficultés dans l'interaction des UDI avec la clientèle (UDI impatients, qui ne veulent pas attendre leur tour, etc.), 15 cas de difficultés relatives à la sécurité du personnel ou des lieux (vol à l'étalage, menaces, etc.), 10 difficultés en matière de gestion du personnel (résistance des pharmaciens ou des autres membres du personnel) et un cas de plainte de l'entourage ${ }^{51}$.

\subsection{Le refus de certains CLSC de récupérer les seringues et les aiguilles usagées}

Plusieurs personnes rencontrées ont aussi déploré le refus de certains CLSC de récupérer les seringues et les aiguilles usagées. Parmi les motifs de refus invoqués par les CLSC eux-mêmes, mentionnons :

- le manque d'espace pour l'entreposage ou la réfrigération des DBM ;

- le manque de ressources humaines et financières ;

- la limite fixée dans la réglementation concernant la quantité maximale de DBM pouvant être transportée sans le recours à une entreprise spécialisée (ce qui serait beaucoup plus coûteux) ;

- l'absence d'équipement (autoclave) pour traiter leurs propres DBM.

Le problème du manque d'espace pour l'entreposage, tout comme celui du manque de ressources humaines et financières, pourrait être réduit si l'on augmentait considérablement le nombre d'endroits où les individus peuvent rapporter leurs seringues et leurs aiguilles usagées.

Le manque d'espace pour la réfrigération ne devrait pas constituer un obstacle à la récupération de seringues usagées de nature domestique. En effet, les Lignes directrices sur la récupération des seringues usagées formulées par le ministère de l'Environnement et de la Faune en mars 1996 stipulent déjà que l'exigence de réfrigération des DBM (art. 22 du Règlement sur les déchets biomédicaux) ne s'applique pas aux programmes de récupération de seringues usagées de nature domestique.

La limite de $50 \mathrm{~kg}$ de DBM par mois pouvant être transportés par l'expéditeur lui-même (ici, le CLSC) jusqu'au lieu d'entreposage ou de traitement constitue un obstacle réel à la récupération pour certains CLSC. À certaines périodes de l'année, p. ex. au moment des campagnes de vaccination, l'ajout de seringues provenant de l'extérieur aux DBM produits par les activités du CLSC lui-même risque fort d'entraîner un dépassement de la limite fixée. Le groupe de travail est

50. Louise VALIQUETTE, Marcel FORTIER et Carole MORISSETTE. Projet pilote de vente et de récupération de seringues en pharmacie pour les utilisateurs de drogues injectables, 1er avril 199430 avril 1995, Montréal, Régie régionale de la santé et des services sociaux de Montréal-Centre, 27 mai 1996, p. 10.

51. DIRECTION DE LA SANTÉ PUBLIQUE DE MONTRÉAL-CENTRE. Services offerts aux UDI dans les pharmacies de Montréal et participation des pharmaciens au projet Stop Sida / UDI-Pharmacies, septembre 1996 - juin 1998, Montréal, Régie régionale de la santé et des services sociaux de Montréal-Centre, septembre 2000, p. 31-32. 
d'avis que cette limite de $50 \mathrm{~kg}$ devrait être rehaussée afin de ne plus représenter un empêchement à la récupération des seringues et des aiguilles usagées pour les CLSC qui font décontaminer leurs DBM dans un autre établissement de santé de leur milieu.

\subsection{La non-disponibilité des autoclaves et le coût de la décontamination et de l'élimination des DBM}

Comme motif pour refuser de récupérer et d'éliminer les seringues et les aiguilles provenant de l'extérieur, des CLSC et des CH invoquent l'obligation de confier leurs propres DBM à un autre établissement public ou de recourir à l'entreprise privée. Plusieurs estiment ne pas être en mesure d'assumer les coûts additionnels que ce service à la communauté représenterait. À notre avis, l'ampleur des difficultés peut être relativisée, compte tenu des quantités de DBM en cause (voir section 3.2) et des tarifs dont bénéficient les établissements de santé publics pour l'élimination de leurs DBM.

Dans la mesure où la limite de $50 \mathrm{~kg}$ de DBM par mois pouvant être transportés sans les contraintes réglementaires serait rehaussée, les CLSC qui font décontaminer leurs propres DBM dans un autre établissement public (ou dans une autre mission du même établissement) pourraient en transporter une quantité plus grande. Ces CLSC reçoivent le service du $\mathrm{CH}$ soit sans frais, soit à prix modique. Quant aux CLSC qui utilisent les services de l'entreprise privée, ils bénéficient des tarifs négociés par les services d'approvisionnement en commun pour les établissements publics de santé ${ }^{52}$. Bien que ces tarifs varient d'une région à l'autre, ils demeurent beaucoup moindres que ceux qui sont généralement appliqués aux petits producteurs privés de DBM tels que cliniques médicales, pharmacies, organismes communautaires et autres. Dans leur cas, compte tenu des volumes en cause, le tarif pour la collecte, le traitement et l'élimination des DBM équivaut parfois à 5 fois et même 10 fois le prix payé par le secteur public pour $1 \mathrm{~kg}$ du même type de déchets.

Les $\mathrm{CH}$ qui ne possèdent pas d'autoclaves bénéficient également des tarifs négociés par les services d'approvisionnement en commun. Dans quatre régions, soit Montréal, Laval, Québec et Chaudière-Appalaches, les $\mathrm{CH}$ ne sont pas équipés d'autoclaves. Dans d'autres régions, certains secteurs couvrant parfois de vastes territoires sont également dépourvus de ces appareils.

Le tableau 6 présente un portrait de la distribution des autoclaves en regard des réseaux locaux de services de santé et de services sociaux nouvellement créés. Il indique aussi le montant approximatif qui, au cours de la dernière année, a été versé à l'entreprise privée pour l'élimination des DBM non anatomiques dans les régions qui ne disposent d'aucun autoclave. Ces montants n'incluent pas le coût des sacs et des boîtes exigés pour le transport ${ }^{53}$.

52. À titre d'exemple, les tarifs actuels pour la collecte, le traitement et l'élimination des DBM non anatomiques (sans bac) sont de $0,55 \$$ le kg pour les établissements publics de Montréal et de Laval et ils sont de 0,57 \$ le kg pour ceux des régions de Québec et de Chaudière-Appalaches.

53. Constitué à partir de données provenant de plusieurs sources, ce portrait est le plus fidèle que nous soyons en mesure de tracer. Il pourrait néanmoins receler quelques inexactitudes. 
Tableau 6

Présence d’autoclaves dans les réseaux locaux de services de santé et de services sociaux (RLSSSS), juillet 2004

\begin{tabular}{|c|c|c|c|}
\hline Région & $\begin{array}{l}\text { Nombre de } \\
\text { réseaux locaux }\end{array}$ & $\begin{array}{l}\text { Nombre } \\
\text { d'autoclaves }\end{array}$ & Remarques \\
\hline 01 Bas-Saint-Laurent & 8 & 8 & 1 autoclave par réseau. \\
\hline 02 Saguenay-Lac-Saint-Jean & 6 & 6 & 1 autoclave par réseau. \\
\hline 03 Québec & 4 & 0 & $\begin{array}{l}4 \text { RLSSSS sont créés : Charlevoix, Portneuf, Québec-Nord et Québec-Sud. } \\
\text { Montant versé à l'entreprise privée l'an dernier : } \pm 250000 \$ \text {. }\end{array}$ \\
\hline 04 Mauricie et Centre-du-Québec & 8 & 8 & $\begin{array}{l}3 \text { autoclaves sur le territoire du RLSSSS Trois-Rivières ; } 2 \text { au même CH à Drummondville. } \\
3 \text { RLSSSS n'en ont pas : Haut-St-Maurice (La Tuque), Vallée-de-la-Batiscan (Saint-Tite), et } \\
\text { Maskinongé (Louiseville). }\end{array}$ \\
\hline 05 Estrie & 7 & 8 & $\begin{array}{l}4 \text { autoclaves sur le territoire du RLSSSS Sherbrooke, } 1 \text { dans chacun des RLSSSS MRC } \\
\text { Memphrémagog (Magog), MRC Granit (Lac Mégantic), MRC Asbestos et MRC Coaticook. } \\
2 \text { RLSSSS n'en ont pas : Haut-Saint-François (Weedon) et Val-Saint-François (Windsor). }\end{array}$ \\
\hline 06 Montréal & 12 & 0 & Montant versé à l'entreprise privée l'an dernier : $\pm 1000000 \$$. \\
\hline 07 Outaouais & 5 & 2 & 2 autoclaves dans le même RLSSSS (Hull-Gatineau). \\
\hline 08 Abitibi-Témiscamingue & 6 & 5 & $\begin{array}{l}1 \text { autoclave par réseau sauf Témiscaming, qui collabore à la fois avec Ville-Marie et avec } \\
\text { l'Ontario. }\end{array}$ \\
\hline 09 Côte-Nord & 8 & 3 & $\begin{array}{l}1 \text { autoclave dans les RLSSSS Sept-Îles et Manicouagan (Baie-Comeau). Celui du CS Basse- } \\
\text { Côte-Nord (Blanc-Sablon) reçoit chaque semaine par bateau les DBM de } 8 \text { dispensaires. } \\
5 \text { RLSSSS n'ont pas d'autoclave. }\end{array}$ \\
\hline 10 Nord-du-Québec & - & 1 & 1 autoclave à Chibougamau. \\
\hline 11 Gaspésie-Îles-de-la-Madeleine & 5 & 5 & 1 autoclave par réseau. \\
\hline 12 Chaudière-Appalaches & 5 & 0 & Montant versé à l'entreprise privée l'an dernier : $\pm 100000 \$ ?$ \\
\hline 13 Laval & 1 & 0 & Montant versé à l'entreprise privée l'an dernier : plus de $76000 \$$. \\
\hline 14 Lanaudière & 2 & 3 & $\begin{array}{l}1 \text { autoclave dans le RLSSSS Lanaudière-Nord (Joliette) et } 2 \text { dans le RLSSSS } \\
\text { Lanaudière-Sud. }\end{array}$ \\
\hline 15 Laurentides & 7 & 6 & $\begin{array}{l}4 \text { RLSSSS ont un autoclave ; } 1 \text { RLSSSS en a } 2 \text { (Mont-Laurier et L'Annonciation); } \\
2 \text { RLSSSS n'en ont pas : Thérèse-de-Blainville et Pays-d'En-Haut (Ste-Adèle). }\end{array}$ \\
\hline 16 Montérégie & 11 & 9 & $\begin{array}{l}1 \text { autoclave sur le territoire de } 9 \text { des } 11 \text { RLSSSS ; } 2 \text { RLSSSS n'en ont pas : Huntingdon et } \\
\text { Presqu'île (Vaudreuil-Dorion). }\end{array}$ \\
\hline 17 Nunavik & - & 1 & $\begin{array}{l}\text { L'autoclave, situé à Kuujjuaq, sert à la décontamination des DBM de } 6 \text { autres villages de } \\
\text { l'Ungaga. Ces DBM sont transportés par avion. }\end{array}$ \\
\hline TOTAL & \multicolumn{3}{|c|}{63 autoclaves dans 55 RLSSSS + 2 (Nord-du-Québec et Nunavik) } \\
\hline
\end{tabular}


Le groupe de travail est d'avis que, dans une perspective non seulement de coût/bénéfice immédiat pour le $\mathrm{CH}$ lui-même, mais également de service à la communauté, les décisions prises il y a 10 ou 15 ans par certains hôpitaux de ne pas s'équiper d'autoclave pourraient être reconsidérées. La mise en place des réseaux locaux de services de santé et de services sociaux nous semble un bon moment pour le faire.

Le coût actuel d'un autoclave pour la décontamination des DBM de modèle 510 avec tiroir (format retenu par le nouvel hôpital Pierre-Le Gardeur de Lachenaie) est de 110000 \$, ce qui comprend : le prix de l'appareil, le transport, la supervision de la mise en marche, la formation d'un utilisateur et une garantie d'une année sur les pièces. Ce montant ne comprend pas les coûts du déchargement de l'appareil, de sa mise en place et des raccordements mécaniques (électricité, vapeur, eau, drainage et ventilation) qui peuvent varier d'un endroit à l'autre selon l'ampleur du travail à exécuter. Il est à noter que ce montant, qui nous a été communiqué en août 2004, n'a pas été obtenu à la suite d'un appel d'offres. Il est présenté ici à titre indicatif seulement. Précisons que la « durée de vie » de ce type d'appareil est d'environ 15 ans.

À la lumière des témoignages recueillis et de nos propres observations, les deux formules, soit posséder son propre autoclave et confier ses DBM non anatomiques à l'entreprise privée, présentent à la fois des avantages et des inconvénients. Le tableau 7 résume ces avantages et inconvénients.

Notre enquête auprès des $\mathrm{CH}$ comportait des questions sur les coûts des différents moyens utilisés pour l'élimination des DBM non anatomiques :

- autoclave sur place (achat des contenants et autres fournitures réglementaires, entreposage et manutention des DBM dans l'établissement, opération et entretien de l'autoclave, transport vers le site d'enfouissement, autres);

- décontamination des DBM dans un autre établissement public (aucun $\mathrm{CH}$ parmi ceux qui ont répondu à l'enquête n'utilisait ce moyen) ;

- recours à l'entreprise privée (achat des contenants et autre matériel d'emballage, entreposage et manutention des DBM dans l'établissement, collecte, traitement et élimination par l'entreprise spécialisée, etc.).

Si le coût des contenants (coût équivalent, quel que soit le moyen utilisé pour l'élimination) et les frais de collecte, traitement et élimination par l'entreprise privée étaient assez faciles à repérer, les autres données étaient beaucoup plus aléatoires. Les coûts des opérations liées au traitement et à l'élimination des DBM sont très souvent inclus parmi les coûts d'entretien sanitaire ou encore d'élimination de l'ensemble des déchets de l'hôpital et il était difficile pour les établissements d'évaluer la proportion du temps de travail du personnel (et donc les coûts) directement liée à la gestion des DBM non anatomiques.

Il n'a donc pas été possible de procéder à une comparaison chiffrée précise des coûts de chacune des formules. Toutefois, compte tenu du coût d'un autoclave (achat, opération et entretien) et de sa durée de vie, il est évident que le recours à l'entreprise privée coûte plus cher que la décontamination des DBM au moyen de l'autoclave de son propre établissement ou d'un autre établissement public. 
Tableau 7

Avantages et inconvénients pour un CH de posséder son propre autoclave ou de confier ses DBM non anatomiques à l'entreprise privée

\begin{tabular}{|c|c|c|c|}
\hline & Autoclave dans l'hôpital & Entreprise privée & Identique \\
\hline $\begin{array}{l}\text { Temps requis pour le tri à la } \\
\text { source (sur les étages) }\end{array}$ & & & $X$ \\
\hline $\begin{array}{l}\text { Temps pour chargement et } \\
\text { déchargement de l'autoclave } \\
\text { et pour nettoyage } \\
( \pm 1 \mathrm{~h} / \text { cycle })\end{array}$ & Requis & Non requis : économie & \\
\hline $\begin{array}{l}\text { Temps pour emballage (sacs } \\
\text { et boîtes) }\end{array}$ & Non requis : économie & Requis & \\
\hline
\end{tabular}

Coût des sacs et boîtes pour

le transport des DBM

piquants et tranchants

Non requis : économie Requis

\begin{tabular}{lll}
\hline Espace pour l'autoclave & Requis & Non requis \\
\hline Espace requis pour & Moindre : on peut lancer un & Plus grand $:$ il faut entrepo- \\
entreposage des DBM & cycle de décontamination & ser les boîtes de contenants \\
& dès que l'autoclave est & et de sacs en attendant le \\
& rempli & moment de la collecte
\end{tabular}

Responsabilité du contrôle

de la qualité de la

décontamination

Assumée par le $\mathrm{CH}$

Pas de responsabilité pour le

$\mathrm{CH}$

Accidents de travail liés à

l'utilisation de l'autoclave

Appareil considéré comme sécuritaire par la très grande majorité des $\mathrm{CH}$ qui l'utilisent

Pas de risque pour les employés du $\mathrm{CH}$

\begin{tabular}{lll}
\hline Achat de l'autoclave & $110000 \$ \div 15=7333 \$ /$ an & Non requis : économie \\
\hline Coût installation autoclave & Variable selon l'endroit & Non requis : économie \\
\hline Coût entretien et réparations & Peu élevé & Aucun : économie \\
\hline
\end{tabular}

Coûts pour

- collecte

Aucun : économie

- traitement

Faible : l'autoclave consomme peu d'énergie

Coût élevé pour les trois opérations

- et élimination

Transport au site

d'enfouissement : requis*

Possibilité de traiter et

éliminer les seringues de

l'extérieur (service à la

Oui, à relativement peu de

frais.

Augmentation des coûts proportionnelle au volume communauté)

* La présence d'objets piquants et tranchants dans les déchets ordinaires de l'hôpital entraîne habituellement une augmentation du montant exigé par le site d'enfouissement. Au moins un $\mathrm{CH}$ a réduit considérablement ses coûts en plaçant les DBM, après décontamination, dans un conteneur différent. Il ne paie le montant additionnel (100 \$ par levée) que pour les conteneurs de DBM. 


\subsection{Le coût des contenants réglementaires}

Le coût des contenants conçus spécifiquement pour les DBM piquants et tranchants constitue un obstacle important à la récupération des seringues et des aiguilles auprès de ceux qui en font un usage domestique. Comme nous l'avons vu précédemment, certains pharmaciens donnent les contenants à leurs clients et les remplacent sans frais lorsqu'ils sont remplis. Cette pratique semble toutefois l'exception. Des CLSC donnent des contenants à certains usagers, mais il ne s'agit certes pas d'une pratique très répandue. Dans le cadre des PES, des contenants sécuritaires sont aussi donnés aux UDI. Là encore, cette approche n'est pas systématique.

Des pharmaciens et des CLSC ont aménagé dans leurs locaux des installations permettant aux individus de déposer eux-mêmes leurs seringues et leurs aiguilles dans le grands contenants réglementaires. Moins onéreuse que la distribution de contenants pour DBM piquants et tranchants aux individus, cette approche implique quand même des coûts que plusieurs ne sont pas disposés à assumer.

Mais, combien coûte au juste un contenant réglementaire pour DBM piquants et tranchants ? Le réseau public de la santé et des services sociaux, par l'intermédiaire des services d'approvisionnement en commun, achète les produits directement du fabricant. Les pharmaciens s'approvisionnent surtout auprès de distributeurs. Deux fabricants ainsi qu'un distributeur ont bien voulu nous faire connaître les prix auxquels ils vendent ces contenants à leurs clients (voir tableau 8). Précisons que ces prix n'ont pas été obtenus au moyen d'un appel d'offres. Ils sont donnés à titre indicatif seulement. Les prix inscrits ici pour le réseau de la santé et des services sociaux sont ceux qui ont été convenus avec Approvisionnements Montréal. Ces prix peuvent varier quelque peu d'une région à l'autre. Les prix de vente du distributeur aux pharmaciens peuvent aussi varier selon les quantités achetées, par exemple par les chaînes et bannières pharmaceutiques. Le prix indiqué ici est le prix courant. Les prix des contenants vendus par MedTech sont ceux qui ont été payés par certains clients de cette entreprise durant la dernière année.

On constate que le prix de vente d'un même produit aux pharmaciens par l'intermédiaire des distributeurs peut représenter le double et même le triple de celui qui est consenti au réseau public de la santé et des services sociaux. Ce à quoi le pharmacien ajoutera sa marge de profit au moment de le vendre au client.

Dans la perspective de rendre les contenants sécuritaires accessibles aux usagers de seringues et d'aiguilles à des fins domestiques, il serait beaucoup plus avantageux d'explorer les solutions qui permettent de les obtenir au prix payé par le réseau public des services de santé et des services sociaux.

À partir des données du tableau 8, on peut calculer, à titre d'exemple, ce qu'il en coûterait par année pour fournir gratuitement des contenants pour DBM piquants et tranchants à une personne diabétique qui requiert 4 injections par jour : 4 injections x 365 jours $=1460$ seringues ou aiguilles.

Étant donné qu'un contenant de 7,6 litres peut renfermer entre 300 et 400 seringues, la récupération sécuritaire des seringues exigerait environ 5 contenants de 7,6 litres, au coût de 4,12 \$ l'unité (prix payé par Approvisionnements Montréal), soit 20,60 \$ par année.

Les lancettes utilisées par ces personnes, qui sont aussi des DBM piquants, trouveraient vraisemblablement leur place dans ces contenants, à travers les seringues et les aiguilles. 
Tableau 8

Comparaison des prix de quelques contenants de récupération pour DBM piquants et tranchants*

\begin{tabular}{|c|c|c|c|c|c|}
\hline Contenant $\mathbf{n}^{0}$ & Volume & $\begin{array}{l}\text { Quantité } \\
\text { ser. } 1 \text { cc* }\end{array}$ & $\begin{array}{l}\text { Caractéristiques } \\
\text { Exemples d'utilisation }\end{array}$ & $\begin{array}{l}\text { Prix } \\
\text { SSS } * *\end{array}$ & $\begin{array}{l}\text { Prix } \\
\text { pharmaciens }\end{array}$ \\
\hline 2300439 & 7,61 & 300 à 400 & $\begin{array}{l}\text { Empilable } \\
\text { Dessus transparent } \\
\text { Col sécuritaire } \\
\text { Ex. : boîtes extérieures, } \\
\text { tatoueurs, acupuncteurs }\end{array}$ & 4,12 & 9,10 \\
\hline BD 30460 & 1,4 & 75 à 90 & $\begin{array}{l}\text { Ouverture sens unique } \\
\text { Non empilable } \\
\text { Col sécuritaire }\end{array}$ & 2,30 & 4,50 \\
\hline TYCO 8906 & 11 & $\pm 60 ?$ & $\begin{array}{l}\text { Couvercle à fermeture } \\
\text { temporaire } \\
\text { Empilable } \\
\text { Populaire en CLSC }\end{array}$ & 1,10 & 3,28 \\
\hline TYCO 8506Y & 51 & 250 à 285 & $\begin{array}{l}\text { Ouverture horizontale à } \\
\text { bascule } \\
\text { Partie du bas empilable } \\
\text { Accepté dans hôpitaux } \\
\text { pour enfants }\end{array}$ & 4,25 & 6,61 \\
\hline BD 300475 & 5,11 & 260 à $300 ?$ & $\begin{array}{l}\text { Ouverture horizontale à } \\
\text { bascule } \\
\text { Peut se fixer au mur avec } \\
\text { support et clef } \\
\text { Bas empilable }\end{array}$ & 3,99 & 7,85 \\
\hline Med-Tech & 4,51 & $\pm 225 ?$ & $\begin{array}{l}\text { Donné par une pharmacie } \\
\text { à ses clients et utilisé par } \\
\text { un service infirmier privé } \\
\text { Non empilable }\end{array}$ & & 3,25 \\
\hline Med-Tech & 251 & & $\begin{array}{l}\text { Chaudière } \\
\text { On peut y déposer des } \\
\text { contenants domestiques } \\
\text { Ex. : org. communautaires }\end{array}$ & & 7,05 \\
\hline $\begin{array}{l}\text { TYCO } \\
8935\end{array}$ & 541 & & $\begin{array}{l}\text { Ouverture coulissante } \\
16 \times 19 \mathrm{~cm} \text {, rouge } \\
\text { Ex. : org. communautaires }\end{array}$ & 16,00 & \\
\hline
\end{tabular}

\footnotetext{
* Notre intention ici n'est pas de recommander l'un ou l'autre de ces contenants, mais uniquement de comparer leur prix respectif.

** Quantité approximative de seringues $1 \mathrm{cc}$ que l'on peut déposer dans le contenant.

*** Prix vendu au réseau de la santé et des services sociaux.
} 
Comme une aiguille pour stylo injecteur occupe environ 5 fois moins d'espace qu'une seringue $1 \mathrm{cc}$, la récupération sécuritaire des aiguilles exigerait environ 1 contenant de 7,6 litres, au coût de $4,12 \$$ par année, ou encore 5 contenants de 1 litre à $1,10 \$$ l'unité, soit 5,50\$.

Pour ce qui est des lancettes utilisées par les personnes atteintes de diabète, mais traitées par d'autres moyens que l'injection d'insuline, sachant qu'une lancette requiert environ 6 fois moins d'espace qu'une seringue $1 \mathrm{cc}$, et que l'on peut déposer environ 60 seringues dans un contenant de 1 litre, un diabétique qui utilise une lancette par jour, aurait besoin d'environ un contenant de ce type par année $(60 \times 6=360)$, au coût de 1,10 \$ l'unité.

Comme l'indique le tableau 9, le coût total des contenants pour DBM requis pour tous les diabétiques québécois au cours d'une année, au tarif payé par le secteur public de la santé et des services sociaux, peut donc être estimé à 739225 \$.

\section{Tableau 9}

\section{Coût des contenants pour DBM requis pour les diabétiques par année}

\begin{tabular}{lccc}
\hline $\begin{array}{l}\text { Type de diabétiques } \\
\text { (format de contenant) }\end{array}$ & $\begin{array}{c}\mathbf{N}^{\text {bre }} \text { contenants } \\
\text { requis par année }\end{array}$ & Prix l'unité en \$ & Coût total en \$ \\
\hline $\begin{array}{l}\text { Diabétiques qui s'injectent de } \\
\text { l'insuline (contenant 7,6 1 pour } \\
\text { seringues, aiguilles et lancettes) }\end{array}$ & $120693^{*}$ & 4,12 & 497225 \\
\hline $\begin{array}{l}\text { Diabétiques qui ne s'injectent pas } \\
\text { d'insuline (contenant 1 1 pour } \\
\text { lancettes) }\end{array}$ & $220000^{* *}$ & 1,10 & 242000 \\
\hline TOTAL & & & 739225 \\
\hline
\end{tabular}

* Voir tableau 3.

** Diabétiques traités autrement que par l'injection d'insuline.

\subsection{L'attitude répressive des policiers à l'endroit des UDI}

Les directions de santé publique et des intervenants auprès des UDI de certaines grandes villes ont indiqué que l'attitude répressive des policiers à l'égard des UDI était un obstacle à la mise en place d'un système intégré permettant la récupération et l'élimination de toutes les seringues usagées. Des UDI, craignant d'être interceptés par la police alors qu'ils sont en possession de seringues - même si cela n'a rien d'illégal -, s'empresseraient de se débarrasser de leurs seringues en les jetant là où ils le peuvent, plutôt que de les rapporter à un site d'échange de seringues. Même des seringues stériles fournies par la santé publique sont confisquées par des policiers.

Le sujet a été abordé d'abord avec le représentant de l'Union des municipalités du Québec. Ce dernier voit là une situation où « les positions de la santé publique et celles de la sécurité publique sont en confrontation ». Il souligne que les municipalités peuvent suggérer aux policiers de ne pas garer leur voiture près des sites d'échange de seringues, mais que la Loi de police leur interdit de faire de l'ingérence dans le travail des policiers. Afin de trouver une solution, il préconise la tenue d'une rencontre au sommet réunissant les principaux acteurs : grandes villes, santé publique, corps de police et groupes communautaires, et même les ministres de la Santé, de la Sécurité publique et des Affaires municipales. 
Nous avons aussi échangé sur le sujet avec le représentant de l'Association des directeurs de police du Québec. Ce dernier a fait en sorte que le sujet soit discuté, en juin 2004, lors d'une réunion regroupant une trentaine de directeurs de police, tant des services de police municipaux que de la Sûreté du Québec. Voici les réponses que ce groupe a fournies à deux questions que nous avions posées :

QUESTION 1 - Avoir des seringues sur soi, sans être en possession de drogue, peut-il constituer un motif d'arrestation sous prétexte que cela constituerait un indice que la personne a pu s'adonner à des activités illégales?

RÉPONSE - Non. Les pouvoirs d'arrestation en vertu du code criminel ne le permettent pas. Le droit de fouille est accessoire au pouvoir d'arrestation.

QUESTION 2- La confiscation de seringues stériles est-elle une pratique approuvée ?

RÉPONSE - Non, sauf si cette confiscation permet d'étayer une preuve criminelle pour une infraction sous enquête.

Sur la question du harcèlement des policiers à l'égard des UDI, le directeur général de l'Association des directeurs de police du Québec dit que, selon l'Association canadienne des chefs de police, le fait de poursuivre n'est pas laissé à la discrétion des policiers. « Si le policier a des motifs de croire qu'une personne a commis un acte illégal, il doit poursuivre. » Il précise toutefois que «dans leur formation, les policiers ne sont pas incités à pourchasser les drogués, mais ceux qui ont commis des infractions ».

L'Association des directeurs de police du Québec n'a pas adopté de position officielle sur la question du harcèlement, par exemple les policiers qui garent leur voiture à la porte d'un site d'échange de seringues et surveillent les allées et venues de la clientèle. Le directeur général est d'avis que l'on pourrait demander aux policiers de mettre fin à une telle pratique. Il verrait aussi d'un bon œil « la création d'un petit comité comprenant des délégués du MSSS et de certains corps de police afin d'étudier l'impact des mesures de coercition sur les personnes qui ont des seringues. »

Le groupe de travail est d'avis qu'il faudrait profiter de l'ouverture manifestée tant par l'Union des municipalités du Québec que par l'Association des directeurs de police du Québec pour poursuivre les échanges sur cette question.

\subsection{Le manque de leadership et de coordination}

Plusieurs ont souligné un manque d'intérêt ou de leadership des autorités régionales ou nationales en santé face à la question de la récupération et de l'élimination sécuritaires des seringues et des aiguilles usagées. On note une confusion entre les rôles que doivent assumer les instances du réseau de la santé et des services sociaux, celles du ministère de l'Environnement et les municipalités, notamment. Des clarifications s'imposent également en ce qui a trait aux rôles que doivent jouer respectivement les équipes en maladies infectieuses, en santé au travail et en santé environnementale.

Plusieurs réclament la manifestation d'une volonté ferme de la part du MSSS de régler le problème. Ils souhaitent une politique à l'échelle du Québec, des consignes cohérentes, des 
mandats clairs pour chacun des acteurs concernés, accompagnés des budgets appropriés. Tout cela, pour que cesse l'organisation à la pièce dans chacune des régions ou sous-régions.

Le groupe de travail est d'avis que le ministère de la Santé et des Services sociaux doit, effectivement, assumer un leadership dans ce dossier, appuyé dans les régions par les agences de développement de réseaux locaux de services de santé et de services sociaux. 


\section{Pour la mise en place d'un système intégré de récupération et d'élimination de toutes les seringues et de toutes les aiguilles usagées}

Le nombre de piqûres accidentelles qui surviennent chaque année, notamment chez les enfants et chez certaines catégories de travailleurs, de même que les coûts croissants de ces accidents incitent le groupe de travail à recommander la mise en place d'un système intégré de récupération et d'élimination de toutes les seringues et de toutes les aiguilles usagées. Ce système devra permettre de surmonter les différents obstacles qui ont été mentionnés au chapitre 8 .

\subsection{Le modèle général proposé}

En tenant compte des commentaires et suggestions recueillis au cours de nos consultations ainsi que des expériences positives réalisées dans divers milieux, nous proposons un modèle général d'organisation comportant les éléments suivants :

- l'application du Règlement sur les déchets biomédicaux à tous ceux qui font usage de seringues et d'aiguilles, y compris ceux qui en font un usage domestique ;

- des modifications au Règlement afin de faciliter la récupération des seringues et des aiguilles auprès des individus ;

- des consignes simples, les mêmes pour tous les utilisateurs de seringues et d'aiguilles à des fins domestiques ;

- l'utilisation généralisée des contenants réglementaires ;

- un accès facilité aux contenants réglementaires ;

- des lieux désignés où l'on serait tenu de récupérer les seringues et les aiguilles usagées ;

- des moyens accessibles pour la décontamination et l'élimination des DBM ;

- une campagne d'information adaptée aux différents publics visés ;

- la conciliation santé publique et sécurité publique.

Ce mode d'organisation fait appel à la collaboration des différents acteurs concernés. Il exigera en outre l'affirmation du leadership du ministère de la Santé et des Services Sociaux en ce domaine et l'indispensable collaboration du ministère de l'Environnement.

Le tableau 10 indique les endroits où les différents types d'utilisateurs de seringues et d'aiguilles, de même que ceux qui en font la récupération auprès des individus ou en trouvent dans l'environnement, pourraient s'en débarrasser de façon sécuritaire. 


\section{Tableau 10}

Endroits où pourraient être acheminés les DBM piquants et tranchants, selon le modèle proposé

\begin{tabular}{|c|c|c|c|c|c|c|c|c|}
\hline \multirow[t]{2}{*}{ Source des objets piquants et tranchants } & \multicolumn{8}{|c|}{ Endroits où ils peuvent apporter leurs DBM piquants et tranchants } \\
\hline & Pharmacie & CLSC & $\begin{array}{l}\text { Organisme } \\
\text { commu- } \\
\text { nautaire }\end{array}$ & $\begin{array}{l}\text { Clinique } \\
\text { spécialisée }\end{array}$ & $\begin{array}{l}\text { Collecteur } \\
\text { dans un } \\
\text { lieu public }\end{array}$ & $\begin{array}{l}\text { CH avec } \\
\text { autoclave }\end{array}$ & $\begin{array}{l}\text { CH sans } \\
\text { autoclave }\end{array}$ & Med-Tech \\
\hline Individus (autosoins, UDI, etc.) & Sans frais & Sans frais & Sans frais & Sans frais & Sans frais & & & \\
\hline $\begin{array}{l}\text { Seringues trouvées par des individus dans } \\
\text { l'environnement* }\end{array}$ & Sans frais & Sans frais & Sans frais & Sans frais & Sans frais & & & \\
\hline Tatoueurs, perceurs, électrolystes & & Sans frais & & & & & & \\
\hline $\begin{array}{l}\text { Seringues récupérées dans l'environnement par } \\
\text { des services publics (petites quantités) }\end{array}$ & & Sans frais & & & & & & \\
\hline $\begin{array}{l}\text { Seringues récupérées par OSBL (refuges pour } \\
\text { itinérants, maisons de jeunes, etc.) }\end{array}$ & & Sans frais & & & & & & \\
\hline $\begin{array}{l}\text { Seringues récupérées dans l'environnement par } \\
\text { services publics (grandes quantités) }\end{array}$ & & & & & & Sans frais & & $\begin{array}{c}\text { Avec } \\
\text { paiement }\end{array}$ \\
\hline $\begin{array}{l}\text { Organismes qui récupèrent auprès des individus } \\
\text { et qui bénéficient des tarifs négociés par les } \\
\text { services d'achat en commun du secteur public } \\
\text { (p. ex. CLSC) }\end{array}$ & & & & & & Sans frais & & $\begin{array}{c}\text { Avec } \\
\text { paiement }\end{array}$ \\
\hline $\begin{array}{l}\text { Organismes qui récupèrent auprès des individus } \\
\text { mais ne bénéficient pas des tarifs négociés par } \\
\text { les services d'achat en com-mun du secteur } \\
\text { public (pharmacies, OC) }\end{array}$ & & & & & & Sans frais & Sans frais & $\begin{array}{c}\text { Avec } \\
\text { paiement }\end{array}$ \\
\hline $\begin{array}{l}\text { Services privés qui utilisent ces objets à des fins } \\
\text { professionnelles (cliniques médicales, dentistes, } \\
\text { acupuncteurs, thanatologues, vétérinaires, } \\
\text { centres d'hébergement, etc.) }\end{array}$ & & & & & & $\begin{array}{l}\text { À prix } \\
\text { modique }\end{array}$ & & $\begin{array}{c}\text { Avec } \\
\text { paiement }\end{array}$ \\
\hline Établissements publics de santé & & & & & & $\begin{array}{l}\text { Sans frais ou } \\
\text { à prix } \\
\text { modique }\end{array}$ & & $\begin{array}{c}\text { Avec } \\
\text { paiement }\end{array}$ \\
\hline $\begin{array}{l}\text { Établissements privés accessibles au public } \\
\text { (hôtels, restaurants, etc.) }\end{array}$ & & & & & & $\begin{array}{l}\text { Sans frais ou } \\
\text { à prix } \\
\text { modique }\end{array}$ & & $\begin{array}{l}\text { Avec } \\
\text { paiement }\end{array}$ \\
\hline
\end{tabular}

* Il peut s'agir d'une ou de quelques seringues, à l'occasion. Toute clinique médicale serait aussi tenue de les accepter. 


\section{2 Étendre l'application du Règlement sur les déchets biomédicaux à tous ceux qui font usage de seringues et d'aiguilles}

Dans sa formulation actuelle, le Règlement sur les déchets biomédicaux prescrit des obligations à ceux qui offrent des soins médicaux, dentaires ou vétérinaires, aux laboratoires de biologie médicale ou vétérinaire et à ceux qui exercent la thanatopraxie. Il ne semble pas couvrir les activités d'acupuncture et il ne s'applique certes pas aux tatoueurs, aux perceurs et aux électrolystes. De plus, il exclut expressément les DBM provenant d'activités domestiques (autosoins, usage de drogues par injection et autres).

Dans divers domaines, l'État impose des contraintes aux individus pour assurer la sécurité des populations ou la protection de l'environnement. Il en est ainsi de l'obligation de porter la ceinture dans un véhicule automobile ou de l'imposition d'un droit environnemental à l'achat de pneus neufs afin d'en assurer la récupération et le recyclage. Dans le même esprit, le groupe de travail estime que l'élimination sécuritaire des déchets biomédicaux que sont les seringues et les aiguilles usagées devrait être obligatoire pour tous et que toute dérogation à cette règle devrait être sanctionnée.

C'est pourquoi il recommande

1. Que le champ d'application du Règlement sur les déchets biomédicaux soit étendu à tous les producteurs de déchets biomédicaux provenant tant de l'usage domestique que des activités professionnelles ${ }^{54}$.

\subsection{Modifier le Règlement afin de faciliter la récupération des seringues et des aiguilles auprès des individus}

Les Lignes directrices sur la récupération des seringues usagées prévoient déjà des assouplissements à l'application du Règlement sur les déchets biomédicaux afin de faciliter la réalisation des programmes de récupération des seringues usagées de nature domestique. Elles suppriment l'obligation de réfrigérer les DBM devant être transportés et elles réduisent les exigences relatives à la tenue du registre de production.

Un obstacle à la récupération demeure en ce qui a trait au transport. Des CLSC et des pharmacies sont susceptibles de récupérer auprès des individus (diabétiques, UDI ou autres) des quantités de seringues et d'aiguilles qui, ajoutées aux DBM provenant de leurs activités régulières, s'élèveront à $50 \mathrm{~kg}$ ou plus par mois. Or, le règlement fixe à moins de $50 \mathrm{~kg}$ par mois la quantité de DBM pouvant être transportée sans que l'on soit tenu de respecter toutes les exigences du Règlement relatives au transport : permis, véhicule réservé, réfrigéré, etc., ce qui augmente sensiblement les coûts. Si cette exigence demeure inchangée, ceux qui sont disposés à faire la récupération de seringues et d'aiguilles auprès des individus et qui ont la possibilité de les apporter à un hôpital muni d'un autoclave, pour décontamination et élimination, pourraient être empêchés de le faire.

Une limite a sans doute été fixée pour éviter que tout un chacun s'improvise transporteur de DBM. Cependant, cette limite semble arbitraire : en quoi serait-il plus dangereux de transporter

54. On trouvera à l'annexe $\mathrm{V}$ la liste des modifications proposées au Règlement sur les déchets biomédicaux. 
$59 \mathrm{~kg}$ de DBM piquants et tranchants dans des contenants réglementaires plutôt que 49 ? En quoi y aurait-il plus de risque à transporter $49 \mathrm{~kg}$ de DBM deux fois par mois plutôt qu'une fois par mois?

Sans déterminer la façon de le faire (augmenter la limite par mois, augmenter la limite par année, transformer la limite en moyenne ou autre chose), le groupe de travail recommande

2. Que soit supprimée du Règlement sur les déchets biomédicaux la limite de moins de $50 \mathrm{~kg}$ de DBM par mois pouvant être transportés sans satisfaire à toutes les exigences (permis, camion réservé exclusivement à cette fin, réfrigération, système de désinfection, etc.) de telle sorte qu'elle ne puisse constituer un obstacle au transport de tous les DBM piquants et tranchants récupérés auprès des individus.

Par ailleurs, l'obligation de réfrigérer les DBM devant être transportés « à l'extérieur de leur lieu de production », dont sont exemptés les organismes qui font la récupération des seringues auprès des individus, s'applique aux autres producteurs de DBM (cliniques médicales, dentistes, etc.), de même qu'à ceux qui reçoivent ces DBM pour le traitement et l'élimination (p. ex. CH muni d'un autoclave qui reçoit les seringues et les aiguilles récupérées par une pharmacie).

Nous avons constaté que cette disposition du règlement est méconnue d'un grand nombre de « petits producteurs » comme les cliniques médicales, les dentistes, les acupuncteurs, etc. Elle est donc peu appliquée dans ces milieux. Elle est cependant bien connue des hôpitaux et pourrait constituer pour eux une raison de refuser d'éliminer avec leurs propres DBM les seringues et les aiguilles récupérées par les CLSC, les pharmacies, les organismes communautaires, etc.

Or, à l'hôpital, les DBM piquants et tranchants, déposés dans des contenants en plastique rigide, passent parfois des semaines dans les chambres des patients, à la température de la pièce. Les couvercles des contenants, une fois fermés, ne peuvent plus être rouverts. Il nous semble peu logique d'exiger qu'au lieu d'entreposage de l'hôpital, ils soient réfrigérés en attendant d'être décontaminés à l'autoclave sur place ou d'être transportés par une entreprise privée spécialisée qui en fera le traitement et l'élimination.

Pour ces raisons, le groupe de travail recommande

3. Que soit supprimée du Règlement sur les déchets biomédicaux toute obligation de réfrigérer les DBM piquants et tranchants.

\subsection{Donner des consignes simples, les mêmes pour tous les utilisateurs de seringues et d'aiguilles à des fins domestiques}

Plus une consigne est simple, claire et générale, plus elle a des chances d'être suivie. Prenons comme exemple l'alcool au volant. Selon le Code de la sécurité routière, quiconque conduit un véhicule routier avec une alcoolémie supérieure à 80 milligrammes d'alcool par 100 millilitres de sang peut se voir retirer sur-le-champ son permis de conduire pour une période de 30 jours (art. 202.4). Cela s'applique à tous, peu importe que la personne ait à franchir une distance de 
$5 \mathrm{~km}$ ou de 50 kilomètres; peu importe qu'elle conduise son véhicule pour une promenade dominicale ou pour des raisons professionnelles.

De la même façon, l'élimination non sécuritaire des déchets biomédicaux met en danger la santé et la sécurité des autres. La consigne devrait être la même pour tous :

Il est interdit de jeter des seringues et des aiguilles usagées dans un réseau d'égout, dans les ordures ménagères et dans les bacs de recyclage.

Les seringues et les aiguilles usagées doivent être déposées dans un contenant pour DBM piquants et tranchants et apportées à la pharmacie, au CLSC, à une clinique privée spécialisée dans le traitement de maladies qui requièrent des autosoins au moyen de seringues et d'aiguilles) ou à un organisme communautaire engagé dans la récupération de seringues

ou encore

être déposées dans un collecteur extérieur de seringues.

La consigne étant la même pour tous, il serait plus facile de produire des outils d'information et de sensibilisation adaptés à un large public. De plus - avantage important - la personne vue en possession d'un contenant de seringues usagées ne serait plus considérée automatiquement comme une "droguée ", avec tous les préjugés que cela comporte. Celui qui rapporte des seringues ou des aiguilles à l'un des endroits désignés serait tout simplement un citoyen responsable.

Le groupe de travail recommande donc

4. Que le Règlement sur les déchets biomédicaux interdise formellement de jeter des seringues et des aiguilles usagées dans un réseau d'égout, dans les ordures ménagères et dans les bacs de recyclage.

5. Que les consignes soient les mêmes pour tous les usagers de seringues et d'aiguilles à des fins domestiques : les seringues et les aiguilles usagées doivent obligatoirement être déposées dans un contenant pour DBM piquants et tranchants et apportées à la pharmacie, au CLSC, à une clinique privée spécialisée dans le traitement de maladies qui requièrent des autosoins au moyen de seringues ou d'aiguilles ou à un organisme communautaire engagé dans la récupération de seringues, ou encore déposées dans un collecteur extérieur de seringues.

Dans le même esprit, l'autorisation accordée aux dentistes de jeter leurs DBM non anatomiques aux ordures ménagères, après les avoir décontaminés sur place au moyen de leur stérilisateur pour instruments réutilisables, devrait être retirée. S'il est interdit aux citoyens qui utilisent des seringues et des aiguilles à des fins domestiques de les jeter aux ordures ménagères, il serait difficile de justifier qu'une catégorie de professionnels de la santé le fasse, même si ces DBM ont été décontaminés au préalable. L'Ordre des dentistes du Québec s'est d'ailleurs montré ouvert à cette idée. 
Le groupe de travail recommande donc

6. Que l'autorisation accordée aux dentistes de jeter leurs DBM non anatomiques aux ordures ménagères, après les avoir décontaminés sur place au moyen de leur stérilisateur pour instruments réutilisables, soit révoquée.

\subsection{Généraliser l'utilisation des contenants réglementaires}

L'analyse présentée au tableau 5 de la section 8.2 montre que les divers types de contenants utilisés pour y déposer les seringues usagées comportent des avantages et des inconvénients. Ceux qui sont le plus sécuritaires, soit les collecteurs pour DBM piquants et tranchants, sont aussi, malheureusement, les plus chers.

Cela va sans dire qu'il est préférable de rapporter des seringues dans un contenant pour pilules (donné par le pharmacien) bien identifié «Déchets biomédicaux piquants et tranchants », ou encore dans une bouteille d'eau de Javel que dans un sac en papier ou en plastique. Toutefois, ces contenants ne résistent pas aussi bien à la perforation que les contenants réglementaires. De plus, la multiplicité des formes et des dimensions des contenants complique la tâche des personnes qui en font la récupération (au CLSC, à la pharmacie, à la clinique spécialisée, dans un organisme communautaire) et de ceux qui en font la manutention (entreposage, transport et traitement). Cela augmente le risque qu'un contenant en plastique opaque ou en métal rempli de seringues mais non identifié se retrouve au recyclage. Les contenants de toutes dimensions et de tous formats sont plus difficile à empiler et à transporter. Enfin, certains de ces contenants fondent durant le processus de décontamination à l'autoclave et risquent d'endommager l'appareil.

Pour toutes ces raisons et afin de protéger la santé et la sécurité des travailleurs appelés à faire la récupération et la manutention des DBM, le groupe de travail recommande

7. Que tous les utilisateurs d'instruments piquants et tranchants, tant à des fins professionnelles qu'à des fins domestiques, soient tenus de les déposer dans des contenants pour DBM spécifiquement prévus à cette fin.

\subsection{Faciliter l'accès aux contenants réglementaires}

Les contenants réglementaires pour DBM piquants et tranchants coûtent cher. Nombreux sont ceux qui manifestent de la réticence à payer ce prix pour des contenants de déchets. Il semble que personne ne soit disposé à assumer cette dépense, pas plus le client que le pharmacien, pas plus le patient que le CLSC. Or, pour que l'utilisation de ces contenants soit généralisée, il faudra que ceux qui utilisent des seringues et des aiguilles à des fins domestiques puissent se les procurer au moindre prix ou mieux, gratuitement.

Nous avons vu, au chapitre 8, que les prix consentis par les fabricants aux services d'approvisionnement en commun sont deux ou trois fois inférieurs à ceux que paient les pharmaciens qui achètent les contenants auprès d'un distributeur. Cela milite en faveur d'un mode d'acquisition de ces contenants par l'intermédiaire du réseau public de la santé et des services sociaux. 
Après consultation de certains utilisateurs réguliers de tels contenants, le MSSS pourrait choisir un nombre limité de modèles et de formats, par exemple, un moyen et un petit. Ces contenants devraient notamment être empilables, afin de réduire l'espace requis pour leur entreposage, être tous de la même couleur (jaune) afin de faciliter leur identification, être dotés d'un col sécuritaire ou d'un mécanisme permettant une fermeture temporaire et, enfin, être dotés d'un mécanisme assurant une fermeture définitive.

La négociation des prix avec les fabricants pourrait être effectuée pour l'ensemble du Québec par le MSSS (ou son mandataire), ce qui pourrait permettre d'obtenir des prix encore plus avantageux, compte tenu du volume d'achat.

Peut-on envisager que ces contenants soient vendus au prix coûtant, c.-à-d. au prix payé au fabricant? Il serait étonnant que l'on puisse le faire par le réseau des pharmacies, étant donné toutes les procédures administratives que cela exigerait pour un objet valant un ou quelques dollars. Pourrait-on les vendre au prix coûtant par l'intermédiaire des CLSC ? Les services offerts par les CLSC sont habituellement gratuits, mais ces établissements vendent à tout le moins certains vaccins. Cependant, cela exigerait beaucoup de transactions et de formalités administratives pour le CLSC. Néanmoins, le montant exigé, soit le prix coûtant, serait encore trop élevé pour la personne âgée diabétique démunie. Faudrait-il alors créer des exceptions en raison de l'âge ou du niveau de revenu ? Cela compliquerait davantage la procédure. En outre, il serait délicat de vendre le contenant aux personnes diabétiques ou dialysées et de le donner aux UDI.

Vaudrait-il mieux vendre les contenants au prix coûtant (autant que possible), mais obtenir qu'ils soient remboursés par l'assurance médicaments? L'usager paierait alors une fraction du prix, tandis que l'État assumerait le reste de la facture. Ceux qui bénéficient d'assurances privées tenteraient également de faire rembourser le coût des contenants par leurs assurances, mais ils devraient en payer une partie (p. ex. $20 \%$ ). Tout cela exigerait encore beaucoup de paperasse et le montant total payé par les usagers et par l'État s'en trouverait considérablement augmenté.

Dans tous les cas où les contenants réglementaires seraient vendus, il faudrait faire en sorte qu'ils soient détaxés. Autrement, la perception des taxes sur chacun de ces petits montants augmenterait les formalités administratives et rendrait encore plus difficilement acceptable l'idée de les vendre au prix coûtant.

Après avoir examiné ces diverses possibilités, il nous apparaît que, dans la mesure où l'utilisation des contenants réglementaires deviendrait une mesure de protection de la santé publique, et compte tenu des montants en cause (739225 \$ dollars par année pour tous les diabétiques du Québec, tel qu'indiqué au tableau 9, ce à quoi s'ajouterait un certain montant pour les autres utilisateurs de seringues et d'aiguilles pour les autosoins), il serait préférable que les contenants réglementaires soient distribués gratuitement aux individus.

Il existe au moins deux mécanismes suivant lesquels, dans le cadre de programmes de santé publique, des produits pharmaceutiques sont remis gratuitement aux patients : les médicaments contre les infections transmises sexuellement (ITS) et les vaccins liés au programme d'immunisation.

Dans le cas des médicaments contre les ITS, on procède ainsi :

1) le pharmacien achète le médicament ;

2) sur ordonnance médicale, le pharmacien remet le médicament gratuitement au patient, c.-à-d. que ce dernier n'a à payer ni la coassurance, ni la franchise ; 
3) le pharmacien réclame à la Régie de l'assurance maladie du Québec (RAMQ) le coût du médicament, sans marge de profit, ainsi que des honoraires de 7,80 \$ par ordonnance.

Dans le cas des vaccins gratuits, on procède ainsi :

1) le MSSS mandate Approvisionnements Montréal (étant donné son expertise en ce domaine) pour procéder à des appels d'offres, lorsque plusieurs fournisseurs vendent le même produit, ou à des ententes de gré à gré dans les cas où un seul fournisseur vend le produit recherché ;

2) un dépositaire provincial reçoit tous les vaccins achetés ;

3) les dépôts régionaux (habituellement des pharmacies d'hôpital ou des directions de santé publique) commandent au dépôt provincial les quantités de vaccins requises ;

4) les CLSC et les cliniques médicales commandent au dépôt régional les quantités de vaccins dont ils ont besoin ;

5) les CLSC administrent les vaccins gratuitement. Normalement, les cliniques médicales font de même.

Si les contenants pour DBM étaient remis gratuitement aux patients par les pharmaciens suivant la formule des médicaments contre les ITS, il faudrait éviter que le prix des contenants se trouve multiplié, soit par des honoraires versés aux pharmaciens pour remettre les contenants à leurs clients, soit par des honoraires versés aux médecins pour des consultations demandées dans le seul but de se faire prescrire un contenant. Le problème est sans doute facilement contournable pour 80000 diabétiques, en liant le contenant à la prescription d'insuline. Cependant, 220000 autres diabétiques utilisent des lancettes (qui deviennent des DBM) en vente libre pour leurs tests de glycémie, mais sont traités autrement que par l'injection d'insuline. Auraient-ils besoin d'une prescription médicale pour le contenant ? Il faudrait également déterminer si les UDI, qui peuvent obtenir du matériel d'injection sans ordonnance à la pharmacie, pourraient obtenir les mêmes contenants sans ordonnance.

Pour demander aux pharmaciens d'offrir ce service (entreposer, puis donner les contenants) sans aucuns honoraires, on pourrait invoquer notamment que ce service n'exige aucune opération financière, qu'il n'implique ni analyse de dossier, ni conseils à donner, ni interaction médicamenteuse à surveiller.

L'application d'un mécanisme inspiré de celui qui est en usage pour les vaccins gratuits serait plus simple au moins sous deux aspects : d'une part, aucune prescription médicale ne serait requise et, d'autre part, les exigences pour le transport et l'entreposage de boîtes en plastique seraient infiniment plus simples que celles qui sont imposées pour la manutention et la conservation des vaccins (p. ex. réfrigération). Les contenants pour DBM pourraient être distribués tant par les pharmaciens que par les CLSC. Et pourquoi pas par les organismes communautaires et les cliniques spécialisées?

D'autres formules pourraient sans doute être imaginées ou explorées, l'objectif visé étant d'offrir sans frais des contenants réglementaires pour DBM à tous ceux qui utilisent des instruments piquants et tranchants pour des autosoins, selon le procédé le moins coûteux possible pour l'État et, au bout du compte, pour la collectivité. Il appartiendra au MSSS, s'il retient cette recommandation, de déterminer le meilleur moyen à utiliser pour atteindre l'objectif fixé. 
Le groupe de travail recommande donc

8. Que le MSSS ou l'un de ses mandataires négocie avec les fabricants l'achat d'un nombre limité de modèles et de formats de contenants pour DBM piquants et tranchants répondant aux besoins des utilisateurs de seringues et d'aiguilles pour les autosoins.

9. Que ces contenants soient distribués gratuitement par les pharmaciens et/ou les CLSC.

\subsection{Désigner des lieux où l’on serait tenu de récupérer les seringues et les aiguilles usagées}

Même si tous ceux qui font usage de seringues et d'aiguilles sont sensibilisés à la nécessité de les éliminer de façon sécuritaire et même si tous ont accès à des contenants réglementaires, encore faudra-t-il qu'ils disposent d'un endroit où ils pourront s'en débarrasser.

Nous l'avons vu, certains pharmaciens et certains CLSC acceptent déjà de récupérer les seringues et les aiguilles usagées. Certains d'entre eux donnent même les contenants réglementaires à leurs patients. Toutefois, comme plusieurs intervenants consultés nous l'ont dit, on ne saurait compter uniquement sur la générosité des différents acteurs pour que toutes les seringues et toutes les aiguilles soient récupérées de façon sécuritaire.

Le groupe de travail croit qu'en multipliant le nombre d'endroits désignés pour la récupération des seringues et des aiguilles, on atteindra à la fois deux objectifs :

- les utilisateurs de ces instruments à des fins domestiques seront incités à les rapporter, puisqu'il se trouvera nécessairement à proximité un endroit où leurs contenants réglementaires remplis seront acceptés sans frais ;

- ceux qui offriront le service de récupération n'hériteront pas d'une charge de travail trop lourde puisque celle-ci sera répartie entre plusieurs acteurs.

Tout comme il est naturel de rapporter les canettes et les bouteilles à l'épicerie, les pneus hors d'usage au garage et les restes de peinture à la quincaillerie, les lieux désignés pour rapporter les seringues et les aiguilles devraient être des endroits naturellement fréquentés par les utilisateurs.

Il serait irréaliste de croire qu'un service de transport puisse faire la collecte des contenants de seringues et d'aiguilles à domicile. Les quantités en cause sont insuffisantes. Les éco-centres ne constituent pas une solution non plus : ils sont trop peu nombreux (Montréal n'en compte que cinq) et les déchets biomédicaux y sont refusés.

Il n'y a pas lieu de désigner comme endroit de récupération de seringues et d'aiguilles tous les cabinets de professionnels où l'on fait usage d'instruments piquants et tranchants. Chacun conviendra qu'il serait inapproprié de demander à la population d'apporter ses seringues et ses aiguilles usagées à la maison funéraire ou chez le vétérinaire. Ceux qui produisent de très petites quantités de DBM piquants et tranchants (dentistes, acupuncteurs, tatoueurs, perceurs et électrolystes) ne vendent pas de seringues ni d'aiguilles et ils ne traitent pas de maladies qui requièrent l'usage de seringues ou d'aiguilles à domicile. 
Certains centres hospitaliers ont installé des contenants de récupération soit à la réception, soit aux urgences, soit dans certains locaux accessibles au public (p. ex. toilettes) où quiconque peut déposer ses seringues et ses aiguilles. Plusieurs $\mathrm{CH}$ munis d'autoclaves acceptent aussi de décontaminer et d'éliminer (souvent sans frais) les DBM que leur apportent les pharmacies, les CLSC et les organismes communautaires qui, eux, les récupèrent auprès des individus. À l'exception des cliniques spécialisées dans le traitement de certaines maladies telles que le diabète, la sclérose en plaques et l'insuffisance rénale, qui requièrent des autosoins à l'aide de seringues ou d'aiguilles, il nous semble inapproprié de demander aux $\mathrm{CH}$ de recevoir, de surcroît, les contenants de seringues et d'aiguilles apportés par les individus.

Quant aux cliniques médicales privées avec ou sans rendez-vous, il semble peu réaliste de leur demander d'offrir le service de récupération de seringues et d'aiguilles à ceux qui en font un usage domestique. D'ailleurs, en 2003-2004, seulement deux cliniques privées (en Montérégie) et une clinique universitaire (en Estrie) étaient inscrites à la liste officielle des centres d'accès aux seringues pour les UDI, au Québec. L'organisation matérielle et l'organisation du travail dans ces cliniques nous semblent peu propices à l'ajout de ce service. Nous croyons cependant raisonnable de demander aux cliniques médicales privées de sensibiliser leurs patients qui font usage de seringues ou d'aiguilles à la nécessité de les éliminer de façon sécuritaire. Aucune clinique ne devrait, non plus, refuser de récupérer et d'éliminer avec ses propres DBM une seringue qu'une personne apporterait après l'avoir trouvée dans l'environnement.

Le groupe de travail est d'avis que les endroits suivants devraient être désignés comme lieux de récupération sans frais des seringues et des aiguilles provenant d'un usage domestique :

\section{- les $\operatorname{CLSC}^{55}$}

En 2004, on comptait 147 CLSC au Québec. Plusieurs d'entre eux avaient plusieurs points de service. Selon la Loi sur les services de santé et les services sociaux, le CLSC doit offrir notamment des services de nature préventive. Certains des services déjà offerts, par exemple en toxicomanie et en dépistage du VIH-sida, touchent des personnes qui peuvent être utilisatrices de seringues ou d'aiguilles. En 2003-2004, 187 points de service des CLSC étaient inscrits à la liste officielle des centres d'accès aux seringues.

La description des services généraux offerts par les centres de santé et de services sociaux inclut «des interventions ayant pour objet la distribution et la récupération de matériel pour injection sécuritaire auprès des personnes qui font usage de drogues par injection et la récupération de seringues usagées trouvées ou utilisées par la population ${ }^{56} \gg$.

Des CLSC acceptent les aiguilles usagées des tatoueurs et des perceurs. Le groupe de travail est d'avis que cette pratique doit être encouragée et qu'elle devrait s'étendre aux électrolystes. En assurant un contact avec ces petits producteurs de DBM dont la pratique n'est pas encadrée par un ordre professionnel, les CLSC pourraient connaitre leurs besoins, par exemple en matière de techniques de désinfection, et leur fournir un minimum d'information (au moyen de dépliants,

55. Dans le cadre de la réorganisation du réseau de la santé et des services sociaux, les CLSC disparaîtront en tant qu'établissements autonomes. Toutefois, la mission qu'ils accomplissent demeurera certainement. Étant donné l'incertitude qui existe concernant la façon dont on désignera finalement cette mission, nous avons maintenu l'appellation CLSC pour nos recommandations.

56. MINISTÈRE DE LA SANTÉ ET DES SERVICES SOCIAUX, Les services généraux offerts par les centres de santé et de services sociaux, Québec, ministère de la Santé et des Services sociaux, septembre 2004, p.51. 
brochures ou autres) sur les moyens de prévenir les infections et, éventuellement, divers traumatismes susceptibles de laisser des séquelles permanentes.

Le service de récupération et d'élimination des seringues usagées devrait aussi être offert par les CLSC aux organismes communautaires (p. ex. refuges pour itinérants, maisons de jeunes, groupes de soutien en toxicomanie) qui, sans faire partie des PES, récupèrent parfois des seringues dans leurs locaux ou aux alentours. Précisons que la contribution exigée des CLSC ne s'étendrait pas à la récupération des seringues et des aiguilles utilisées à des fins professionnelles par tous les petits producteurs privés de DBM (soins infirmiers à domicile, centres d'hébergement privés, etc.), pas plus que les CLSC ne seraient tenus de leur fournir les contenants réglementaires.

Plus des deux tiers des CLSC qui ont répondu à notre enquête considèrent que la récupération, l'entreposage et l'élimination sécuritaires de seringues provenant de l'extérieur (UDI, PES, autosoins et seringues trouvées dans l'environnement) sont des responsabilités que devraient assumer les CLSC en général. Dans les mêmes proportions, ils réclament l'augmentation ou même l'existence de budgets à cette fin. Rappelons à ce sujet que les CLSC peuvent soit acheminer leurs DBM non anatomiques pour traitement et élimination aux hôpitaux munis d'autoclaves (qui, dans bien des cas, font maintenant partie du même établissement), soit recourir aux services de l'entreprise privée, en bénéficiant des tarifs négociés par les services d'approvisionnement en commun.

- les cliniques spécialisées dans le traitement de certaines maladies

Les cliniques spécialisées dans le traitement de certaines maladies telles que le diabète, la sclérose en plaques et l'insuffisance rénale sont généralement intégrées à un centre hospitalier ou à un centre de santé qui produit lui-même de grandes quantités de DBM. Les $\mathrm{CH}$ peuvent déjà compter sur du personnel affecté à la gestion de ces DBM. De plus, les CH sont équipés d'un autoclave ou ils confient leurs DBM à l'entreprise privée spécialisée en bénéficiant des tarifs convenus avec les services d'approvisionnement en commun. Dans le contexte où les contenants réglementaires seraient fournis gratuitement aux patients, il nous semble raisonnable d'exiger que les cliniques spécialisées dans le traitement de maladies qui requièrent des autosoins au moyen de seringues et d'aiguilles soient tenues d'offrir le service de récupération et d'élimination des seringues usagées.

- les pharmacies communautaires

On trouve actuellement au Québec quelque 1600 pharmacies communautaires (c.-à-d. autres que celles des hôpitaux). Certaines d'entre elles comptent plusieurs points de service. Cela fait de la pharmacie un lieu facilement accessible pour la plupart des gens. Normalement, c'est à la pharmacie que les patients achètent les seringues, les aiguilles et les lancettes qu'ils utilisent pour leurs autosoins. Ils y achètent également leurs médicaments injectables.

Certains pharmaciens offrent déjà le service de récupération des seringues à leur clientèle, souvent sans le publiciser. Tous les pharmaciens que nous avons rencontrés se reconnaissent une certaine responsabilité dans la récupération des seringues et des aiguilles. Cependant, la plupart considèrent qu'ils n'ont pas à assumer les coûts des contenants pour DBM piquants et tranchants ni les coûts de collecte, de traitement et d'élimination de ces DBM. La récupération des médicaments périmés, à laquelle ils sont tenus de collaborer par leur code de déontologie, est une 
responsabilité qu'ils jugent déjà lourde. Par ailleurs, certains ont des réticences à s'afficher comme un lieu où les UDI peuvent apporter leurs seringues, pour des raisons déjà énoncées.

Tant l'Ordre des pharmaciens du Québec que l'Association québécoise des pharmaciens propriétaires considèrent que « ceux qui vendent des seringues devraient les récupérer ».

Dans le contexte où les contenants réglementaires seraient fournis gratuitement aux patients et dans la mesure où les pharmaciens n'auraient pas à assumer les frais d'élimination des seringues et des aiguilles récupérées, le groupe de travail estime que tous les pharmaciens devraient être tenus d'accepter les seringues et les aiguilles usagées qui leur seraient apportées par les individus, clients ou non.

- les organismes communautaires engagés dans l'échange de seringues

Les organismes communautaires qui oeuvrent auprès des UDI offrent déjà, avec le soutien des directions de santé publique, le service de récupération des seringues. Ils déploient des efforts immenses pour inciter les UDI à rapporter leurs seringues usagées. Certains leur fournissent des contenants réglementaires dans différents formats répondant à leurs besoins. Quelques-uns ont installé des collecteurs de seringues à l'extérieur de leurs locaux.

Des organismes communautaires nous ont dit qu'ils recevaient parfois des seringues apportées par des diabétiques qui ne trouvaient pas d'endroit où s'en débarrasser de façon sécuritaire. La responsable d'un salon de tatouage nous a aussi dit qu'elle apportait ses contenants d'aiguilles usagées à un organisme communautaire.

Il est entendu que les organismes communautaires qui oeuvrent auprès des UDI continueront à offrir des services essentiellement à cette clientèle. Nous estimons cependant qu'ils ne devraient pas refuser les seringues ou les aiguilles utilisées à d'autres fins domestiques (p. ex. autosoins).

- Les collecteurs de seringues installés dans des lieux publics

Des collecteurs de seringues sont installés dans divers lieux publics, surtout à Montréal, qui en compte 25. L'entretien de ces collecteurs est sous la responsabilité respectivement de la Ville de Montréal (7), de l'UQAM (3), d'organismes communautaires (7), de CLSC (6), d'un pharmacien et de la Direction de santé publique de Montréal. On trouve quelques-uns de ces collecteurs dans d'autres villes, par exemple, à l'entrée d'un hôpital ou près des locaux d'un organisme communautaire. D'autres municipalités ont manifesté leur ouverture ou leur intérêt à ce que de tels équipements soient installés aux endroits problématiques.

Plusieurs personnes consultées représentant des milieux aussi diversifiés que les pharmacies, les organismes communautaires, les directeurs de police et Services médicaux Med-Tech ont suggéré l'augmentation du nombre de collecteurs de seringues dans les lieux publics comme moyen de réduire le nombre de seringues qui se retrouvent à des endroits inappropriés.

Ce dispositif n'est toutefois pas une panacée. Pour une utilisation optimale, il doit être installé à un endroit approprié. Il faut qu'une personne soit responsable de son entretien: changer les contenants et ramasser les seringues qui peuvent se retrouver à l'extérieur du contenant, le tout en 
respectant les normes de sécurité, en plus de remplir le formulaire de suivi ${ }^{57}$. De plus, il arrive à l'occasion que certains collecteurs de seringues soient vandalisés. À la lumière des expériences vécues ici et ailleurs, il y aurait lieu de vérifier si des dispositifs plus robustes pourraient être utilisés.

Quiconque utilise des seringues ou de aiguilles à des fins domestiques devrait pouvoir les apporter à l'un ou l'autre de ces cinq endroits sans devoir expliquer ou justifier quoi que ce soit. Dans l'ensemble, on peut s'attendre à ce que les organismes communautaires engagés dans l'échange de seringues continuent de recevoir essentiellement des seringues usagées des UDI. Les cliniques spécialisées recevront sans doute uniquement des seringues et des aiguilles apportées par des patients qui y sont traités. Les pharmacies recevront surtout des seringues provenant de leur clientèle qui s'y procure ses médicaments et son matériel d'injection ainsi que les seringues usagées d'un certain nombre d'UDI, surtout là où l'on vend ou donne du matériel d'injection sans ordonnance. Les CLSC, eux, recevront des DBM piquants et tranchants provenant de diverses sources. Quant aux collecteurs de seringue installés dans des lieux publics, ils devraient être de moins en moins associés uniquement aux UDI.

Les personnes qui trouvent des seringues dans l'environnement devraient aussi pouvoir les apporter à ces endroits. À cette fin, les CLSC, les cliniques spécialisées, les pharmacies et les organismes communautaires devraient toujours être munis d'un grand contenant réglementaire permettant d'y déposer le contenant (souvent non réglementaire) dans lequel des seringues trouvées dans l'environnement auraient été apportées. Ce grand contenant réglementaire devrait aussi être mis à la disposition des individus qui, surtout durant la phase de transition vers l'usage généralisé des contenants réglementaires, apporteront des seringues dans des contenants de fortune.

L'installation de contenants réglementaires pour DBM piquants et tranchants devrait aussi être encouragée chez les propriétaires ou gestionnaires de lieux privés accessibles au public (p. ex. hôtels, restaurants, bars). Ce serait un moyen peu coûteux pour eux d'éviter les piqûres accidentelles chez leur personnel ou leur clientèle de même que les autres frais pouvant résulter d'une mauvaise gestion des seringues et des aiguilles (p. ex. seringues jetées dans les toilettes).

Le Groupe de travail recommande donc

10. Que soient désignés comme lieux où les utilisateurs de seringues et d'aiguilles à des fins domestiques peuvent s'en débarrasser gratuitement et de façon sécuritaire :

- les CLSC (ou autre appellation de cette mission dans les centres de santé et de services sociaux),

- les cliniques spécialisées dans le traitement de maladies qui requièrent des autosoins au moyen de seringues ou d'aiguilles,

- les pharmacies communautaires,

- les organismes communautaires engagés dans l'échange de seringues,

- les collecteurs de seringues installés dans des lieux publics.

57. Voir à ce sujet le document Recommandations pour l'installation et l'utilisation de la boîte de récupération de seringues usagées produit par la Direction de santé publique de Montréal-Centre dans le cadre des travaux du comité intersectoriel sur la réduction des seringues à la traîne, juin 2002. 
11. Que tous ces établissements, services ou organismes, de même que les cliniques médicales privées, soient tenus de récupérer et d'éliminer sans frais avec leurs propres DBM les seringues trouvées dans l'environnement apportées occasionnellement par des individus.

12. Que les CLSC soient aussi tenus de recevoir et d'éliminer sans frais avec leurs propres DBM les instruments piquants et tranchants provenant des activités de tatouage, de perçage et d'électrolyse de même que les seringues récupérées par les organismes communautaires tels que refuges pour itinérants, maisons de jeunes et groupes de soutien en toxicomanie et celles qui sont trouvées en petite quantité dans l'environnement par des services publics (CPE, écoles, municipalité, police ou autres).

13. Que les municipalités, les établissements publics de santé, les établissements d'enseignement, les centres sportifs, les maisons de jeunes et les autres services publics ou communautaires situés dans des secteurs où l'on trouve fréquemment des seringues dans l'environnement soient invités à examiner, en collaboration avec les ressources en santé publique au besoin, la pertinence d'installer sur leur terrain un ou des collecteurs de seringues usagées. (N. B. Chaque organisme ou établissement sera responsable de fournir, installer et entretenir ces équipements.)

14. Que les propriétaires ou gestionnaires de lieux privés accessibles au public (p. ex. hôtels, restaurants, bars) soient invités à installer aux endroits appropriés des collecteurs de seringues à l'intention de leur clientèle.

\subsection{Faciliter l'accès aux équipements collectifs pour la décontamination et l'élimination des DBM}

Ceux qui rendent service à la société en récupérant les seringues et les aiguilles usagées auprès des individus ne doivent pas être obligés d'assumer, de surcroît, les frais du traitement et de l'élimination de ces DBM. Actuellement, deux services existent pour la décontamination des DBM non anatomiques: les autoclaves que l'on trouve dans une soixantaine d'hôpitaux et l'entreprise Services médicaux Med-Tech.

Plusieurs hôpitaux munis d'autoclaves - payés par les fonds publics - acceptent de décontaminer et d'éliminer des DBM non anatomiques provenant de l'extérieur. Le groupe de travail estime que tous les hôpitaux équipés de ce type d'appareil devraient accepter de le faire sans frais pour les CLSC, les cliniques spécialisées dans le traitement du diabète et autres, les pharmacies et les organismes communautaires qui récupèrent les seringues et les aiguilles usagées auprès des individus, de même que pour les services municipaux ou autres services publics qui récupèrent des seringues dans l'environnement.

Par ailleurs, en acceptant de décontaminer et d'éliminer à prix modique les DBM provenant d'autres services professionnels (cliniques médicales, dentistes, thanatologues, acupuncteurs, centres d'hébergement privés, etc.), ces hôpitaux peuvent compenser les frais encourus par le traitement gratuit des seringues et des aiguilles récupérées auprès des individus, tout en empêchant que certains utilisateurs professionnels soient tentés de se débarrasser de leurs DBM de façon non sécuritaire pour éviter de débourser les montants exigés par l'entreprise privée. 
Le groupe de travail est d'avis que, dès maintenant, les hôpitaux qui ne possèdent pas d'autoclave et qui recourent aux services de l'entreprise privée devraient quand même accepter de recevoir et d'éliminer sans frais avec leurs propres DBM les seringues et les aiguilles récupérées auprès des individus par ceux qui ne bénéficient pas des tarifs négociés par les services d'approvisionnement en commun (soit les pharmacies, certains organismes communautaires et, éventuellement, certaines cliniques spécialisées.). Bien entendu, ceux qui récupèrent des seringues et des aiguilles auprès des individus mais qui désirent continuer de recourir aux services de l'entreprise privée pourraient continuer de le faire, mais dans ce cas ils devraient en défrayer eux-mêmes les coûts.

Dans certaines régions, on trouve déjà dans chaque réseau local de services de santé et de services sociaux un établissement doté d'un autoclave (voir tableau 6). Étant donné les économies qui pourraient être réalisées par les hôpitaux eux-mêmes, et vu l'importance de cet équipement pour le service à la communauté, les hôpitaux qui ne sont pas munis d'autoclave devraient évaluer, avec les agences de développement des réseaux locaux de services de santé et de services sociaux, la possibilité de se doter de cet appareil. Dans les régions où les réseaux locaux couvrent de petits territoires, des ententes pourraient exister afin qu'un même hôpital muni d'un autoclave reçoive et élimine les seringues récupérées auprès des individus sur plus d'un territoire de réseau local.

Selon nous, les $\mathrm{CH}$ n'ont pas à craindre d'être "enterrés » sous les DBM provenant de l'extérieur. Des données datant du début des années 90 indiquent que les hôpitaux génèrent près de $85 \%$ des déchets biomédicaux au Québec. Les autres producteurs se partageraient les $15 \%$ qui restent ${ }^{58}$. Les proportions ont-elles changé depuis ce temps ? Impossible de répondre avec certitude à cette question. Par exemple, avec le virage ambulatoire, les autosoins exigeant l'usage de seringues et d'aiguilles à la maison sont plus nombreux. En même temps, l'usage de plus en plus répandu du stylo injecteur au lieu des seringues conventionnelles, chez les diabétiques, réduit le volume de DBM provenant de cette source. Aucune méthode nouvelle ne semble avoir révolutionné les pratiques des médecins, des dentistes ou des laboratoires au point de modifier sensiblement les quantités de seringues et d'aiguilles requises. Par contre, on peut croire que l'incinération des cadavres, de plus en plus répandue, réduit les volumes de DBM piquants et tranchants provenant des thanatologues. De leur côté, les programmes d'échange de seringues, mis en place en vue de prévenir la transmission du VIH et des hépatites, ont vraisemblablement contribué à augmenter le volume de seringues en circulation chez les usagers de drogues par injection.

Les diminutions compenseraient-elles les augmentations ? Un indice nous est fourni par les enquêtes que nous avons menées auprès des hôpitaux. Parmi les hôpitaux munis d'autoclaves qui offrent le service de décontamination des DBM non anatomiques à de nombreux « clients » tels que pharmacies, CLSC, cliniques médicales, dentistes, acupuncteurs, thanatologues et autres, la proportion de DBM provenant de l'extérieur qu'ils traitent est généralement inférieure à $15 \%$ de leur volume total. En voici des exemples :

- CH Pierre-Boucher, Longueuil : 31 clients, moins de $7 \%$ de DBM provenant de l'extérieur ;

- Hôtel-Dieu de Saint-Jérôme : 51 clients, moins de $2 \%$ de DBM provenant de l'extérieur ;

- Centre hospitalier de Rouyn-Noranda : 21 clients, incluant ceux qui récupèrent les seringues auprès des UDI et des diabétiques, moins de $5 \%$ de DBM provenant de l'extérieur.

58. Cette donnée est mentionnée notamment dans la présentation du Règlement sur les déchets biomédicaux, Le règlement en bref, site Web du ministère de l'Environnement. 
Dans les circonstances, il est raisonnable de croire que, même si tous les DBM non anatomiques d'une région aboutissaient à l'hôpital de cette région, la proportion de DBM provenant de l'extérieur de l'hôpital ne dépasserait pas $15 \%$.

Rendre ce service entraînerait-il des difficultés majeures pour l'hôpital ? Dans notre enquête auprès des $\mathrm{CH}$, nous avons posé la question suivante "Ce service [décontaminer au moyen de votre autoclave des seringues usagées provenant de l'extérieur et les éliminer] que vous rendez à des utilisateurs de l'extérieur vous cause-t-il des problèmes?» À cette question, 31 établissements ont répondu «Cela ne nous cause aucun problème." Les autres ont mentionné les problèmes suivants :

- surcroît de travail important pour le personnel (3) ;

- surcroît (ou léger surcroît) de travail pour le personnel (5) ;

- augmentation des accidents de travail $(1)^{59}$;

- augmentation significative des coûts (2);

- légère augmentation des coûts (1).

Quelques-uns ont aussi mentionné des problèmes liés à la négligence de certains utilisateurs extérieurs (contenants inadéquats, mal identifiés ou laissés à des endroits inappropriés).

En somme, le service peut être rendu à la communauté sans inconvénient majeur.

Afin que ce service à la communauté - rendu dans le but d'éliminer si possible les piqûres d'aiguilles accidentelles «dans la communauté »- ne devienne pas la cause d'accidents chez le personnel préposé à la manutention et au traitement des DBM dans les hôpitaux, ces derniers devraient s'assurer que leur personnel reçoive une formation adéquate et qu'il bénéficie d'un environnement et de conditions de travail sécuritaires.

Par ailleurs, les lacunes dans le tri des déchets à la source ont parfois des incidences sur le volume de DBM à traiter et sur les coûts du traitement et de l'élimination. Une attention spéciale devrait être portée au tri à la source des DBM tant par les « clients » extérieurs que par les hôpitaux euxmêmes.

Le groupe de travail recommande donc

15. Que tous les hôpitaux équipés d'autoclaves acceptent de décontaminer et d'éliminer sans frais les seringues et les aiguilles usagées récupérées auprès des individus par les CLSC, les cliniques spécialisées dans le traitement de maladies qui requièrent des autosoins au moyen de seringues ou d'aiguilles, les pharmacies et les organismes communautaires, de même que celles qui sont récupérées dans l'environnement par les services municipaux ou par d'autres services publics.

59. Des piqûres accidentelles sont survenues parce que des seringues avaient été déposées dans des contenants autres que les contenants réglementaires. Elles n'ont toutefois pas exigé l'application de mesures de prophylaxie. 
16. Que les hôpitaux équipés d'autoclaves soient invités à offrir à prix modique les services de décontamination et d'élimination des DBM non anatomiques provenant d'autres services professionnels (cliniques médicales, dentistes, thanatologues, acupuncteurs, centres d'hébergement privés, etc.), ce qui permettrait de compenser, du moins en partie, les frais encourus par le traitement sans frais des seringues et des aiguilles récupérées auprès des individus.

17. Que les hôpitaux qui ne sont pas munis d'autoclave évaluent, avec l'agence de développement de réseaux locaux de services de santé et de services sociaux, la possibilité de se doter de cet appareil. (N. B. Dans les régions où les réseaux locaux couvrent de petits territoires, des ententes pourraient exister afin qu'un même hôpital muni d'un autoclave reçoive et élimine les seringues et les aiguilles récupérées auprès des individus sur des territoires de réseaux locaux voisins.)

18. Que, dès maintenant, les hôpitaux qui ne possèdent pas d'autoclave soient tenus d'éliminer sans frais avec leurs propres DBM les seringues et les aiguilles récupérées auprès des individus par ceux qui ne bénéficient pas des tarifs négociés par les services d'approvisionnement en commun (soit les pharmacies, certains organismes communautaires et, éventuellement, certaines cliniques spécialisées).

19. Que les hôpitaux s'assurent que leur personnel préposé à la manutention et au traitement des DBM reçoive une formation adéquate et qu'il bénéficie d'un environnement et de conditions de travail sécuritaires.

\subsection{Offrir aux professionnels et aux travailleurs une formation et des mesures de prévention appropriées}

Les piqûres accidentelles et la résistance à participer au processus de récupération des seringues et des aiguilles résultent souvent d'un manque d'information et de formation. Par ailleurs, l'absence de récupération systématique des seringues et des aiguilles amène des individus à s'en débarrasser de façon non sécuritaire, ce qui peut causer des accidents. On peut briser ce cercle vicieux en offrant aux professionnels et aux travailleurs concernés une formation et des mesures de prévention appropriées.

Dans le secteur de la santé et des services sociaux, diverses mesures sont déjà prises pour inciter le personnel soignant qui utilise des instruments piquants et tranchants à adopter des équipements et des méthodes de travail sécuritaires. Il faut en faire une préoccupation constante, étant donné les nombreux accidents qui s'y produisent encore chaque année. Les professionnels de la santé doivent aussi être sensibilisés aux dangers que représentent pour d'autres travailleurs (préposés à l'entretien ménager, personnel de la buanderie, de la stérilisation, etc.) les objets piquants et tranchants laissés à des endroits inappropriés. Ces travailleurs, pour leur part, doivent être prévenus de la présence possible d'instruments piquants et tranchants souillés parmi les objets qu'ils sont appelés à manipuler; ils doivent être informés de la marche à suivre lorsque cela se produit, être incités à adopter des équipements et des méthodes de travail sécuritaires et savoir ce qu'il faut faire advenant une piqûre accidentelle. Des mesures de prévention doivent être offertes aux personnes qui présentent le plus de risques d'être exposées à du sang ou à des liquides biologiques. 
De la formation et des mesures de prévention doivent aussi être proposées aux autres utilisateurs d'instruments piquants et tranchants à des fins professionnelles (acupuncteurs, thanatologues, électrolystes, tatoueurs, perceurs, etc.). Cela, tant pour leur propre protection que pour celle de leur clientèle et des autres travailleurs de leur milieu, de même que pour la protection de ceux qui font la manutention des déchets jusqu'à leur élimination définitive.

D'autres catégories de travailleurs (concierges, agents de sécurité, policiers, éboueurs, préposés à l'entretien des parcs, etc.), tout comme ceux de divers services publics (garderies, écoles, postes, etc.) ou privés (restaurants, hôtels, stations-service, etc.), peuvent aussi se trouver en présence de seringues ou d'aiguilles usagées. Ils doivent recevoir une formation sur les risques que cela représente, sur la façon d'éliminer ces objets de façon sécuritaire et sur les démarches à faire en cas de piqûre accidentelle.

Une attention particulière doit être portée au personnel des endroits désignés pour la récupération des seringues et des aiguilles (pharmacies, CLSC, cliniques spécialisées et organismes communautaires), qui doivent aussi recevoir la formation appropriée et pouvoir bénéficier de mesures de prévention et de prophylaxie postexposition.

Cette formation, de même que la mise en place de mesures de prévention, pourrait être offerte par les équipes en santé et sécurité du travail locales et régionales. Ce personnel est mandaté et payé par la CSST, sur la base de contrats établis entre le MSSS et la CSST. Pour faire en sorte que tous les professionnels et tous les travailleurs concernés puissent bénéficier de la formation et des mesures de prévention appropriées, une entente doit donc être convenue entre ces deux instances.

Pour ces raisons, le groupe de travail recommande

20. Que le ministère de la Santé et des Services sociaux effectue les démarches nécessaires auprès de la CSST afin que les équipes en santé et sécurité du travail soient mandatées pour donner de la formation aux travailleurs et aux professionnels à risque de subir des piqûres accidentelles et pour mettre en place des mesures de prévention appropriées.

21. Que les agences de développement de réseaux locaux de services de santé et de services sociaux et les CLSC, en collaboration avec les employeurs concernés, contribuent à la formation des travailleurs et des professionnels à risque et à la mise en place des mesures de prévention appropriées.

\subsection{Mener une campagne d'information adaptée aux différents publics concernés}

Au cours de notre démarche, nous avons été à même de constater que, à l'exception des hôpitaux et de Services médicaux Med-Tech, rares étaient ceux qui connaissaient avec précision la teneur du Règlement sur les déchets biomédicaux.

La diversité des consignes données concernant la récupération et l'élimination des seringues et des aiguilles usagées crée une grande confusion particulièrement chez ceux qui utilisent ces instruments pour les autosoins. Cette diversité des consignes empêche d'ailleurs la centrale InfoSanté CLSC de diffuser un protocole à ce sujet dans l'ensemble des régions. 
Les divers regroupements nationaux représentant les professionnels de la santé, de même que les associations de malades, se sont montrés disposés à diffuser de l'information sur la réglementation (modifiée) et sur divers aspects de la récupération sécuritaire des seringues et des aiguilles touchant leurs membres ou les usagers de leurs services. Pour ce faire, ils offrent leurs publications, leur site Web, des kiosques d'information à l'occasion de certains événements, etc. Ils se disent prêts à accueillir des ressources du MSSS à des sessions de formation, à des colloques, à des congrès, etc. afin de traiter de ces questions.

Au niveau local, tous ceux à qui nous avons posé la question (pharmaciens, cliniques médicales, municipalités et autres) ont affirmé qu'ils se feraient un plaisir de diffuser, par divers moyens, de l'information adaptée à leur clientèle.

Une représentante de la centrale Info-Santé CLSC a aussi indiqué que, dès que la nouvelle réglementation sera adoptée, et dans la mesure où les consignes seront les mêmes pour toutes les régions, un protocole Info-Santé pourra être élaboré.

Le groupe de travail est d'avis que l'adoption de modifications au Règlement sur les déchets biomédicaux et l'application de nouvelles directives (contenants réglementaires pour tous, endroits où se les procurer, endroits désignés pour se débarrasser des contenants remplis, etc.) doivent faire l'objet d'une campagne d'information qui utilisera les moyens appropriés pour les divers publics :

- utilisateurs de seringues et d'aiguilles à des fins domestiques (interdiction de jeter des DBM dans un réseau d'égout, aux ordures ménagères et les bacs de recyclage, contenants à utiliser, où se les procurer, leur prix s'il y a lieu, comment s'en débarrasser une fois remplis, comment en obtenir d'autres, etc.) ;

- professionnels de la santé : consignes précises pour leurs membres, information à donner aux usagers de leurs services ;

- municipalités : promotion de la récupération des seringues dans les lieux publics, information à leurs employés, consignes aux citoyens concernant les déchets recyclables et ceux qui doivent être mis aux ordures ménagères (p. ex. pictogrammes sur les bacs à ordures et les bacs de recyclage);

- associations de malades et organismes œuvrant auprès des UDI : consignes à donner à leur clientèle sur la nécessité et les moyens de récupérer toutes les seringues, les aiguilles et les lancettes de façon sécuritaire.

Cette campagne d'information devrait être coordonnée par le ministère de la Santé et des Services sociaux, en collaboration avec le ministère de l'Environnement. Elle devrait associer les instances régionales et locales en santé, les directions régionales du ministère de l'Environnement ainsi que les autres acteurs concernés, dont les municipalités, les associations représentant les professionnels de la santé et les associations de malades.

Le groupe de travail recommande donc

22. Que ministère de la Santé et des Services sociaux coordonne, en collaboration avec le ministère de l'Environnement, une campagne d'information sur la récupération sécuritaire des seringues et des aiguilles usagées. 
23. Que les instances régionales et locales en santé, les directions régionales du ministère de l'Environnement ainsi que les autres acteurs concernés, dont les municipalités, les associations représentant les professionnels de la santé et les associations de malades, soient associés à cette campagne d'information.

\subsection{Concilier la protection de la santé publique et la protection de la sécurité publique}

Les représentants de municipalités que nous avons consultés, de même que l'Association des directeurs de police, se sont montrés sensibles aux conséquences que peuvent avoir sur la protection de la santé publique les pratiques de certains policiers qui, par exemple, vont garer leur voiture près d'un site d'échange de seringues afin de surveiller les allées et venues de la clientèle.

D'une manière générale, tout en assumant leurs fonctions dans le domaine de la sécurité publique, ils ne souhaitent pas annuler les efforts déployés par les directions de santé publique et les organismes communautaires afin de réduire le partage de matériel d'injection et favoriser la récupération des seringues.

Dans ce contexte, le groupe de travail est d'avis qu'il faut profiter de l'ouverture et de l'intérêt manifestés par ces acteurs dans le dossier pour poursuivre le dialogue et en arriver à des approches qui seront complémentaires dans la recherche du bien-être des populations. C'est pourquoi il recommande

24. Que le ministère de la Santé et des Services sociaux prenne l'initiative de réunir des représentants des corps policiers, des municipalités, de la santé publique et des organismes communautaires afin de trouver les moyens les plus adéquats de concilier les actions de protection de la santé publique en matière de récupération des seringues usagées et celles qui visent la protection de la sécurité publique.

\subsection{2 Élaborer une politique nationale de récupération des seringues et des aiguilles}

Nous l'avons vu, la mise en place d'un système intégré pour la récupération de toutes les seringues et de toutes les aiguilles usagées au Québec exige la contribution de nombreux partenaires. Au cours de nos consultations, nous avons été à même de constater que la plupart d'entre eux se reconnaissaient un rôle et des responsabilités dans la recherche de solutions. Plusieurs ont exprimé le souhait que le ministère de la Santé et des Services sociaux assume un leadership dans ce dossier. On souhaite des orientations claires, des consignes précises, une définition des rôles de chacun et les ressources nécessaires.

Dans cette perspective, le groupe de travail recommande

25. Que le ministère de la Santé et des Services sociaux élabore une politique nationale de récupération des seringues et des aiguilles usagées, qu'il exerce un leadership afin de mobiliser les partenaires concernés, qu'il propose des mesures concrètes, des moyens pratiques (qui doit faire quoi, comment) et qu'il alloue les ressources nécessaires pour la mise en place du système de récupération et d'élimination sécuritaires des seringues et des aiguilles usagées. 


\subsection{Réunir les acteurs concernés dans chacune des régions}

Aucune région n'a déjà mis en place un système intégré tel que nous le proposons, incluant l'utilisation des contenants sécuritaires par tous les usagers de seringues et d'aiguilles, tant à des fins domestiques que professionnelles. Toutefois, nous ne partons pas à zéro. Loin de là.

On trouve minimalement des centres d'accès aux seringues pour les UDI dans chacune des régions, où l'on récupère et assure l'élimination des seringues. Dans au moins une région, on récupère systématiquement les seringues et les aiguilles des diabétiques et des dialysés. Une soixantaine d'hôpitaux sont déjà munis d'autoclaves (dans certains cas, un par réseau local de services de santé et de services sociaux). Plusieurs offrent déjà le service de décontamination et d'élimination des DBM non anatomiques à différents établissements publics, à des services privés ou publics, à des organismes communautaires. Des régions ont établi des mécanismes de coordination entre la Direction de la santé publique, les CLSC, les municipalités, les services policiers, les organismes communautaires, etc. À certains endroits, on a installé des collecteurs de seringues dans des lieux publics. Et, partout, on trouve de la bonne volonté.

Il appartient à chaque région d'élaborer un état de la situation et, tenant compte des réalités et des besoins du milieu, de formuler un projet de système intégré mettant à contribution les partenaires concernés. L'agence de développement de réseaux locaux de services de santé et de services sociaux nous semble l'instance la mieux placée pour prendre l'initiative de réunir ces partenaires et solliciter leur aide de diverses façons : distribuer des contenants réglementaires, récupérer les seringues et les aiguilles auprès des individus qui en font un usage domestique, éliminer sans frais les seringues et les aiguilles ainsi récupérées, installer des collecteurs de seringues, donner de la formation, diffuser de l'information, etc.

C'est pourquoi le groupe de travail recommande

26 Que, dans le cadre d'une politique nationale québécoise, les agences de développement de réseaux locaux de services de santé et de services sociaux prennent l'initiative de réunir les acteurs concernés pour mettre en place un système intégré pour la récupération de toutes les seringues et toutes les aiguilles usagées sur chaque territoire de réseau local de services de santé et de services sociaux de la région.

Des questions qui ne sont pas directement liées à la récupération et à l'élimination des seringues et des aiguilles usagées ont été soulevées par certains de nos interlocuteurs au cours de nos consultations (voir annexe VI). Comme ces questions ne font pas partie de notre mandat, nous ne les avons pas approfondies et nous n'avons pas formulé de recommandations à leur sujet. Nous croyons néanmoins utile de les signaler aux autorités du MSSS. 



\section{Partager les responsabilités}

$\mathrm{Au}$ terme de sa réflexion, le groupe de travail a acquis la conviction que la récupération et l'élimination sécuritaires des seringues et des aiguilles usagées ne peut reposer uniquement sur les individus, ni sur un seul acteur ou uniquement quelques-uns d'entre eux. Il s'agit d'une responsabilité à partager. Cela exigera des modifications aux comportements des individus, mais également des changements dans les pratiques de certains professionnels et de certains établissements.

Afin que chaque acteur individuel ou collectif puisse saisir quelle part de responsabilités lui reviendrait dans l'hypothèse où le modèle général que nous proposons serait retenu, nous présentons une liste des principales actions qui seraient attendues de chacun. Certaines actions qui sont déjà inscrites dans les pratiques habituelles de certains acteurs ou qui font consensus parmi les acteurs concernés sont mentionnées à titre de rappel.

Il est bien entendu que cette liste de responsabilités n'interdit à personne de faire plus ou mieux.

\subsection{Les individus}

- Ne pas jeter leurs seringues, leurs aiguilles et leurs lancettes dans un réseau d'égout, dans les ordures ménagères ou dans les bacs de recyclage, mais les déposer dans un contenant pour déchets biomédicaux (DBM) piquants et tranchants qu'ils pourront se procurer gratuitement à la pharmacie ou au CLSC.

N.B. La personne qui trouvera une seringue à un endroit inapproprié pourra, bien sûr, la déposer dans un contenant de plastique ou de métal résistant à la perforation et muni d'un couvercle solide.

- Rapporter les contenants à la pharmacie, au CLSC, à une clinique spécialisée dans le traitement de maladies qui requièrent des autosoins au moyen de seringues ou d'aiguilles, à un organisme communautaire engagé dans l'échange de seringues, ou encore les déposer dans un collecteur de seringues installé dans un lieu public.

\subsection{Les CLSC}

- Mettre à la disposition de leur clientèle de l'information produite par le MSSS sur la façon de se débarrasser des seringues et des aiguilles (consignes claires et simples).

- Distribuer des contenants réglementaires aux usagers de seringues et d'aiguilles à des fins domestiques.

- Recevoir et assurer l'élimination sans frais des seringues et des aiguilles utilisées avec ou sans ordonnance médicale apportées par des individus.

- Éliminer sans frais avec leurs propres DBM les instruments piquants et tranchants provenant des activités de tatouage, de perçage et d'électrolyse de même que les seringues récupérées par les organismes communautaires tels que refuges pour itinérants, maisons de jeunes, groupes de soutien en toxicomanie et celles qui sont trouvées en petite quantité dans l'environnement par des services publics (CPE, écoles, municipalité, police ou autres).

- Mettre à la disposition de la clientèle un collecteur grand format permettant aux usagers d'y déposer eux-mêmes les seringues et les aiguilles usagées (trouvées dans l'environnement ou 
provenant de l'usage domestique) qui seraient apportées dans des contenants non réglementaires, surtout durant la phase de transition vers l'usage généralisé des contenants réglementaires.

N. B. Il ne devrait y avoir aucune manipulation de seringues et d'aiguilles usagées par le personnel.

- CLSC avec mission en santé et sécurité du travail: en collaboration avec les employeurs concernés, contribuer à la formation des professionnels et des travailleurs à risque et à la mise en place des mesures de prévention appropriées.

- En collaboration avec la Direction de santé publique, diffuser de l'information à la population en général et à certains publics particuliers (UDI, diabétiques, écoliers, etc.)

\subsection{Les pharmaciens}

- Tout pharmacien qui vend des seringues ou des aiguilles est tenu d'en faire la récupération.

- Mettre à la disposition de leur clientèle de l'information produite par le MSSS sur la façon de se débarrasser des seringues et des aiguilles (consignes claires et simples).

- Distribuer des contenants réglementaires aux usagers de seringues et d'aiguilles à des fins domestiques

- Recevoir et assurer l'élimination sans frais des seringues et aiguilles utilisées avec ou sans ordonnance médicale apportées par des individus.

- Mettre à la disposition de la clientèle un collecteur grand format permettant aux usagers d'y déposer eux-mêmes les seringues et les aiguilles usagées (trouvées dans l'environnement ou provenant de l'usage domestique) qui seraient apportées dans des contenants non réglementaires, surtout durant la phase de transition vers l'usage généralisé des contenants réglementaires.

N. B. Il ne devrait y avoir aucune manipulation de seringues et d'aiguilles usagées par le personnel.

- Apporter les seringues et les aiguilles récupérées auprès des individus à un centre hospitalier (qui, lui, sera tenu de les recevoir et de les éliminer sans frais). Autrement, l'élimination sera faite aux frais du pharmacien.

\subsection{Les hôpitaux équipés d'autoclaves}

- Décontaminer et éliminer sans frais les seringues et les aiguilles usagées récupérées auprès des individus par les CLSC, les cliniques spécialisées, les pharmacies et les organismes communautaires, de même que celles qui sont récupérées dans l'environnement par les services municipaux ou par d'autres services publics.

- Offrir à prix modique les services de décontamination et d'élimination des DBM non anatomiques provenant d'autres services professionnels (cliniques médicales, dentistes, thanatologues, acupuncteurs, centres d'hébergement privés, etc.), ce qui permettra de compenser, du moins en partie, les frais encourus par le traitement sans frais des seringues et des aiguilles récupérées auprès des individus. 


\subsection{Les hôpitaux non pourvus d'autoclaves}

- Recevoir et éliminer sans frais avec leurs propres DBM les seringues et les aiguilles récupérées auprès des individus par ceux qui ne bénéficient pas des tarifs négociés par les services d'approvisionnement en commun (soit les pharmacies, certains organismes communautaires et, éventuellement, certaines cliniques spécialisées).

- Évaluer avec l'agence de développement de réseaux locaux de services de santé et de services sociaux, la possibilité de se doter d'un autoclave. (N. B. Dans les régions où les réseaux locaux couvrent de petits territoires, des ententes pourraient exister afin qu'un même hôpital muni d'un autoclave reçoive et élimine les seringues et les aiguilles récupérées auprès des individus sur des territoires de réseaux locaux voisins.)

\subsection{Tous les hôpitaux}

- S'assurer que leur personnel préposé à la manutention et au traitement des DBM reçoive une formation adéquate et qu'il bénéficie d'un environnement et de conditions de travail sécuritaires.

- Porter une attention spéciale au tri à la source des DBM tant par les hôpitaux eux-mêmes que par leurs « clients » extérieurs pour le service de décontamination et d'élimination des DBM.

\subsection{Les cliniques spécialisées}

- Mettre à la disposition de leur clientèle de l'information produite par le MSSS sur la façon de se débarrasser des seringues et des aiguilles (consignes claires et simples).

- Recevoir et assurer l'élimination sans frais des seringues et aiguilles utilisées avec ou sans ordonnance médicale apportées par des individus.

- Mettre à la disposition de la clientèle un collecteur grand format permettant aux usagers d'y déposer eux-mêmes les seringues et les aiguilles usagées (trouvées dans l'environnement ou provenant de l'usage domestique) qui seraient apportées dans des contenants non réglementaires, surtout durant la phase de transition vers l'usage généralisé des contenants réglementaires.

N. B. Il ne devrait y avoir aucune manipulation de seringues et d'aiguilles usagées par le personnel.

- Si la clinique n'est pas située dans un centre hospitalier, apporter les seringues et les aiguilles récupérées auprès des individus à un centre hospitalier (qui, lui, sera tenu de les recevoir et de les éliminer sans frais). Autrement, l'élimination sera faite aux frais de la clinique.

\subsection{Les médecins}

- Mettre à la disposition de leur clientèle de l'information produite par le MSSS sur la façon de se débarrasser des seringues et des aiguilles (consignes claires et simples). 


\subsection{Les ordres professionnels, les corporations et les associations professionnelles}

- Diffuser de l'information sur le Règlement sur les déchets biomédicaux à leurs membres.

- Dans le cadre d'inspections professionnelles, surveiller les méthodes utilisées par leurs membres pour l'élimination des DBM.

\subsection{Les organismes communautaires}

- Poursuivre la sensibilisation de leur clientèle à la nécessité d'utiliser des contenants réglementaires et de rapporter les seringues usagées aux endroits désignés, soit à la pharmacie, au CLSC, à une clinique spécialisée dans le traitement de maladies qui requièrent des autosoins au moyen de seringues ou d'aiguilles, à un organisme communautaire engagé dans l'échange de seringues, ou encore de les déposer dans un collecteur de seringues installé dans un lieu public.

- S'assurer que les travailleurs de rue disposent de l'équipement approprié pour transporter les seringues usagées de façon sécuritaire.

\subsection{Les associations de malades}

- Sensibiliser leurs membres et les usagers de leurs services à la nécessité d'utiliser des contenants réglementaires et de rapporter les seringues, aiguilles et lancettes usagées aux endroits désignés, soit à la pharmacie, au CLSC, à une clinique spécialisée dans le traitement de maladies qui requièrent des autosoins au moyen de seringues ou d'aiguilles, à un organisme communautaire engagé dans l'échange de seringues, ou encore de les déposer dans un collecteur de seringues installé dans un lieu public.

\subsection{Les municipalités}

- En collaboration, au besoin, avec les ressources en santé publique (CLSC ou DSP) et les organismes communautaires, installer et entretenir des collecteurs de seringues dans les lieux publics, aux endroits appropriés.

- Fournir à leur personnel (préposés à la collecte des ordures ménagères, à l'entretien des rues et des parcs, etc.) la formation et l'équipement nécessaires pour leur sécurité.

- Fournir aux citoyens l'information concernant l'interdiction de jeter des seringues et des aiguilles dans un réseau d'égout, dans les ordures ménagères et dans les bacs de recyclage.

\subsection{Les services policiers}

- Doter les voitures de police de l'équipement nécessaire pour récupérer des seringues usagées de façon sécuritaire et indiquer aux policiers la façon de s'en débarrasser.

- Avec le ministère de la Santé et des Services sociaux, les municipalités, les directions de santé publique et les organismes communautaires, contribuer à la recherche des moyens les plus adéquats de concilier les actions de protection de la santé publique en matière de récupération des seringues usagées et celles qui visent la protection de la sécurité publique. 


\subsection{Les agences de développement de réseaux locaux de services de santé et de services sociaux}

- Dans le cadre d'une politique nationale québécoise, prendre l'initiative de réunir les acteurs concernés pour mettre en place un système intégré pour la récupération de toutes les seringues et de toutes les aiguilles usagées sur chaque territoire de réseau local de services de santé et de services sociaux de la région.

- En collaboration avec les municipalités et les autres employeurs concernés, contribuer à la formation des travailleurs et des professionnels à risque et à la mise en place de mesures de prévention appropriées.

- Faire des démarches auprès des pharmaciens pour les inciter à récupérer les seringues et les aiguilles usagées.

- Faire des démarches auprès des $\mathrm{CH}$ pour les inciter à éliminer sans frais avec leurs propres DBM les seringues et les aiguilles récupérées auprès des individus par les pharmaciens, les CLSC, les organismes communautaires et les cliniques spécialisées ainsi que celles qui sont trouvées dans l'environnement par les divers services publics.

- En collaboration avec les CLSC et les municipalités, diffuser de l'information à la population en général et à certains publics particuliers (UDI, diabétiques, écoliers, clientèle des services personnels, etc.)

- Soutenir les organismes communautaires engagés dans la récupération de seringues.

- Participer à la campagne d'information.

\subsection{Le ministère de la Santé et des Services sociaux}

- Manifester une volonté ferme de régler le problème : élaborer une politique nationale de récupération des seringues et des aiguilles, exercer un leadership, proposer des mesures concrètes, des moyens pratiques (qui doit faire quoi, comment) et y allouer les ressources appropriées.

- Effectuer lui-même ou mandater des partenaires pour effectuer les démarches nécessaires afin que les contenants réglementaires soient fournis gratuitement à tous ceux qui font usage de seringues et d'aiguilles à des fins domestiques.

- Effectuer les démarches nécessaires auprès de la CSST afin que les équipes en santé et sécurité du travail soient mandatées pour donner de la formation aux travailleurs et aux professionnels à risque et pour mettre en place des mesures de prévention appropriées.

- Soutenir les démarches visant l'acquisition d'autoclaves par les $\mathrm{CH}$.

- Coordonner, en collaboration avec le ministère de l'Environnement, une campagne d'information sur la récupération sécuritaire des seringues et des aiguilles usagées. Les instances régionales et locales en santé, les directions régionales du ministère de l'Environnement ainsi que les autres acteurs concernés, dont les municipalités, les associations représentant les professionnels de la santé et les associations de malades, devraient être associés à cette campagne d'information.

- Diffuser régulièrement des mises à jour sur les maladies transmissibles, les risques, les moyens de les prévenir, etc. à l'intention de ceux qui font un usage professionnel de seringues et d'aiguilles à l'extérieur du réseau de la santé et des services sociaux (acupuncteurs, thanatologues, électrolystes, etc.). 


\subsection{Le ministère de l'Environnement}

- Modifier le Règlement sur les déchets biomédicaux. Voir la liste complète des modifications proposées à l'annexe IV.

- Révoquer l'autorisation donnée aux dentistes de mettre aux ordures ménagères les DBM décontaminés au moyen des autoclaves de leur cabinet.

- Collaborer avec le ministère de la Santé et des Services sociaux à une campagne d'information sur la récupération sécuritaire des seringues et des aiguilles usagées et y associer ses directions régionales. 


\section{Conclusion}

Le problème des seringues usagées abandonnées dans l'environnement ou laissées à des endroits inappropriés n'est pas lié uniquement à l'usage de drogues par injection. Les lacunes dans la réglementation, le manque d'information, et surtout l'absence de moyens pour se débarrasser de façon sécuritaire des DBM piquants et tranchants provenant de l'usage domestique et, dans certains cas, d'activités professionnelles, expliquent pour une bonne part la situation actuelle.

Selon nous, il est urgent d'agir car la gestion inadéquate des DBM piquants et tranchants risque de causer de plus en plus d'accidents et de coûter de plus en plus cher au système de santé et de services sociaux et à la société en général. Qu'il suffise de rappeler qu'en neuf ans, l'hôpital Sainte-Justine de Montréal a traité, à lui seul, pas moins de 145 enfants à la suite de "piqûres d'aiguilles accidentelles dans la communauté ». Les accidents de travail impliquant des seringues et des aiguilles acceptés par la CSST augmentent chaque année; ils ont doublé en cinq ans. Une piqûre accidentelle qui survient à l'extérieur du réseau de la santé et des services sociaux, sans séroconversion, coûte en moyenne 1687 \$ uniquement en frais remboursés par la CSST.

Avec le virage ambulatoire, plusieurs maladies, qui auparavant exigeaient l'hospitalisation du patient, sont maintenant traitées à la maison. L'évolution des méthodes de traitement entraîne une recrudescence des autosoins qui requièrent l'utilisation de seringues et d'aiguilles à domicile. À titre d'exemple, on commence plus tôt l'injection d'insuline pour traiter le diabète. Actuellement, une bonne part des DBM qui en résultent prennent la voie des ordures ménagères et, quelquefois, des bacs de recyclage. Une situation que la très grande majorité des organismes et des personnes que nous avons consultés jugent inacceptable.

Pour mettre en place un système intégré de récupération et d'élimination sécuritaires des seringues et des aiguilles usagées, il nous paraît clair que des modifications doivent être apportées au Règlement sur les déchets biomédicaux. Les pratiques de certains établissements et de certains professionnels devront se transformer. Des ressources devront être consenties, notamment pour fournir des contenants réglementaires aux utilisateurs de seringues et d'aiguilles à des fins domestiques (UDI, diabétiques et autres). L'acquisition éventuelle d'autoclaves par certains centres hospitaliers exigera également des investissements à court terme qui pourraient se transformer en économies à long terme. La prévention a un prix, nous le reconnaissons volontiers, mais le prix de la maladie est bien plus élevé encore.

La récupération et l'élimination sécuritaires des seringues et des aiguilles usagées, c'est une responsabilité à partager. Le système que nous proposons suppose la collaboration de nombreux acteurs. À cet égard, des expériences fructueuses de collaboration déjà réalisées dans certains milieux et l'intérêt que la démarche de notre groupe de travail a suscité chez tous les acteurs nous laissent croire que chacun fera sa part le moment venu, dans le cadre d'un plan concerté. En effet, les personnes et les organismes consultés ont été nombreux à souhaiter une politique de santé publique concernant la récupération des seringues et des aiguilles usagées, des mandats clairs pour chacun, des consignes cohérentes pour tous les utilisateurs de seringues et d'aiguilles et des moyens d'information adéquats.

La réorganisation en cours, avec la création des réseaux locaux de services de santé et de services sociaux, peut être une occasion de développer des modes de collaboration entre les établissements publics de santé et les différents acteurs concernés par la récupération des seringues et des aiguilles. Dans les décisions à prendre, il nous paraît essentiel que, en plus des intérêts immédiats de ceux qui produisent les plus gros volumes de DBM, on considère les bénéfices que la 
collectivité pourrait retirer d'une mise en commun des équipements disponibles et d'une collaboration étroite entre tous les acteurs concernés.

Nous souhaitons donc que les autorités du ministère de la Santé et des Services sociaux manifestent une volonté claire de régler ce problème. Qu'elles fassent appel à la collaboration de tous : individus, associations professionnelles, établissements de santé, municipalités, organismes communautaires et autres. Nous sommes persuadés que cet appel sera entendu et que chacun acceptera de contribuer à la protection de la santé et de la sécurité des enfants, des travailleurs et de la population en général, de même qu'à la préservation de la qualité de l'environnement. 


\section{Annexe I}

\section{Des contributions précieuses}

Le Groupe de travail sur la récupération des seringues usagées au Québec a mené trois enquêtes au moyen de questionnaires écrits. Ces enquêtes s'adressaient respectivement aux directions de santé publique, aux CLSC et CHSLD ainsi qu'aux centres hospitaliers. Les DSP et les établissements dont les noms suivent ont répondu au questionnaire qui leur était destiné.

DIRECTIONS DE SANTÉ PUBLIQUE

Direction de la santé publique du Bas-Saint-Laurent

Direction régionale de la santé publique du Saguenay-Lac-Saint-Jean

Direction régionale de santé publique de Québec

Direction de la santé publique de la Mauricie et du Centre-du-Québec

Direction régionale de la santé publique de l'Estrie

Direction de la santé publique de Montréal-Centre

Direction de la santé publique de l'Outaouais

Direction de la santé publique de l'Abitibi-Témiscamingue

Direction de la santé publique de la Côte-Nord

Direction de la santé publique du Nord-du-Québec

Direction de santé publique de la Gaspésie-Îles-de-la-Madeleine

Direction de santé publique de Chaudière-Appalaches

Direction de la santé publique de Laval

Direction de la santé publique de Lanaudière

Direction de la santé publique des Laurentides

Direction de santé publique de la Montérégie

Direction de la santé publique du Nunavik

Direction de la santé publique des Terres cries de la Baie James

\section{CLSC ET CHSLD}

Bas-Saint-Laurent

CLSC Rivières et Marées (Rivière-du-Loup)

CLSC de la Vallée et CH d'Amqui

CLSC de Matane

CLSC-CHSLD Rimouski-Neigette

Réseau de santé et de services sociaux des Basques (Trois-Pistoles)

Centre mitissien de santé et de services communautaires (Mont-Joli)

Saguenay-Lac-Saint-Jean

CLSC du Grand Chicoutimi

CLSC Le Norois (Alma)

Centre Maria-Chapdelaine (Dolbeau-Mistassini)

Carrefour de santé Jonquière

CHSLD de Chicoutimi 
Québec

CLSC-CHSLD Haute-Ville-Des-Rivières (Québec)

CLSC et CHSLD de Charlevoix

CLSC-CHSLD Basse-Ville-Limoilou-Vanier (Québec)

CLSC-CHSLD Sainte-Foy-Sillery-Laurentien (Sainte-Foy)

CLSC-CHSLD La Source (Foyer de Charlesbourg)

Centre de santé de Portneuf (Saint-Marc-des-Carrières)

Centre de santé Orléans (Beaupré)

Mauricie et Centre-du-Québec

CLSC Suzor-Côté (Victoriaville)

CLSC Drummond (Drummondville)

CSSS Vallée-de-la-Batiscan (Saint-Tite)

Centre de santé Cloutier-du Rivage (Cap-de-la-Madeleine)

CSSS de la Saint-Maurice (La Tuque)

Centre de santé les Blés d'or (Fortierville)

Centre de santé de la MRC de Maskinongé (Louiseville)

CSSS de l'Énergie (Shawinigan)

CHSLD Le Trifluvien (Trois-Rivières)

CHSLD Cœur-du-Québec (Drummondville)

CHSLD Ermitage (Victoriaville)

Estrie

CLSC de Sherbrooke

Carrefour de la santé et des services sociaux du Val-Saint-François (Windsor)

Centre de santé de la MRC de Coaticook (Coaticook)

CLSC-CHSLD du Haut-Saint-François (Weedon)

Centre de santé de la MRC d'Asbestos (Asbestos)

Carrefour de santé du Granit (Lac Mégantic)

Institut universitaire de gériatrie de Sherbrooke et CHSLD Estriade

Montréal

CLSC Parc Extension

CLSC du Vieux-Lachine

CLSC René-Cassin

CLSC Saint-Léonard

CLSC du Plateau Mont-Royal

CLSC Lac-Saint-Louis

CLSC Pierrefonds

CLSC Verdun-Côte-Saint-Paul

CLSC Saint-Henri

CLSC Olivier-Guimond

CLSC Villeray

CLSC Côte-des-Neiges 
CLSC des Faubourgs

CLSC Métro

CLSC Rivière-des-Prairies

CLSC Mercier-Est-Anjou

CLSC-CHSLD La Petite Patrie

CLSC-CHSLD de Rosemont

CLSC Ahuntsic et Résidence Laurendeau-Légaré-Louvain

CH-CLSC Lasalle

CLSC-CHSLD Pointe-aux-Trembles-Montréal-Est

CLSC-CHSLD Nord-de-1'Île

CHSLD Lachine Nazaire-Piché et Foyer Dorval

Centre gériatrique Maimonides

CHSLD Foyer Rousselot

CHSLD du Plateau Mont-Royal

CHSLD Lucille-Teasdale

CHSLD Biermans-Triest

CHSLD de Mon Quartier

CHSLD Providence Notre-Dame-de-Lourdes

CHSLD Les Havres

CHSLD Champlain-Manoir de Verdun

Centre d'accueil Dante

CHSLD Juif de Montréal

Manoir Cartierville

Institut universitaire de gériatrie de Montréal

Résidence Yvon-Brunet

Outaouais

CLSC de Hull

CLSC-CHSLD de la Petite-Nation (Saint-André-Avellin)

CLSC Vallée-de-la-Lièvre (Buckingham)

CHSLD de Hull

CH-CHSLD de Papineau (Buckingham)

Abitibi-Témiscamingue

CLSC Le Partage des eaux (Rouyn-Noranda)

Centre de santé Vallée-de-l'Or (Val-d'Or)

CLSC-CHSLD Les Eskers (Amos)

Réseau de la santé et des services sociaux des Aurores boréales (La Sarre)

Centre de santé Sainte-Famille (Ville-Marie)

CHSLD Maison Pie XII (Rouyn-Noranda)

Côte-Nord

CLSC et Centre d'hébergement de Manicouagan (Baie-Comeau)

Centre de santé de l'Hématite (Fermont) 
Gaspésie-Îles-de-la-Madeleine

CLSC des Îles-de-la-Madeleine (Cap-aux-Meules)

CLSC Mer et Montagnes (Rivière-au-Renard)

CLSC-CHSLD Pabok - volet hébergement (Chandler)

Centre hospitalier Baie-des-Chaleurs (Maria)

Chaudière-Appalaches

CLSC Beauce-Sartigan (La Guadeloupe)

CLSC Frontenac (Thetford-Mines)

CLSC-CHSLD de la MRC Desjardins (Lévis)

CLSC-CHSLD de la MRC Montmagny (Saint-Fabien-de-Panet)

CLSC-CHSLD Nouvelle-Beauce (Sainte-Marie)

Centre de santé des Etchemins (Lac-Etchemin)

Centre de santé Arthur-Caux (Laurier-Station)

Centre de santé Paul-Gilbert (Lévis)

CLSC Beauce-Centre (Saint-Joseph-de-Beauce)

Laval

CLSC-CHSLD du Marigot

CLSC des Mille-Îles-CHSLD Laval

CLSC-CHSLD Sainte-Rose de Laval

CLSC-CHSLD du Ruisseau-Papineau

Lanaudière

CLSC-CHSLD d'Autray (Sainte-Élisabeth)

Carrefour de la santé et des services sociaux Matawinie (CLSC-CHSLD) (Chertsey)

CLSC-CHSLD Meilleur (L'Assomption)

CLSC-CHSLD Montcalm (Saint-Esprit)

Laurentides

CLSC Hautes-Laurentides (Mont-Laurier)

CLSC Jean-Olivier-Chénier (Saint-Eustache) et CHSLD de la Rive et Mirabel

CLSC-CHSLD Thérèse-de-Blainville (Sainte-Thérèse)

CLSC-CHSLD des Pays-d'en-Haut (Sainte-Adèle)

CLSC Arthur-Buies (Saint-Jérôme)

Établissement de santé d'Argenteuil (Lachute)

CH-CLSC-CHSLD des Sommets (Mont-Tremblant)

Montérégie

CLSC Samuel-de-Champlain (Brossard)

CLSC Simonne-Monet-Chartrand (ancien CLSC Longueuil-Est)

CLSC Huntingdon

CLSC Kateri (Candiac)

CLSC Seigneurie de Beauharnois (Valleyfield)

CLSC Jardin du Québec (Saint-Rémi) 
Les CLSC et CHSLD de La Pommeraie (Bedford)

CLSC-CHSLD Champagnat de la Vallée des Forts (Iberville)

CLSC du Richelieu (Marieville)

CLSC-CHSLD de la MRC d'Acton (Acton Vale)

CHSLD du Bas-Richelieu (Sorel)

CHSLD Trèfle d'Or (La Prairie)

Le Regroupement des CHSLD des Trois Rives (Vaudreuil-Dorion)

Hébergement Longueuil - Les CHSLD de Longueuil

CENTRES HOSPITALIERS

Bas-Saint-Laurent

Centre hospitalier régional de Rimouski

Réseau de santé du Témiscouata (Notre-Dame-du-Lac)

Réseau de santé Kamouraska (Saint-Pascal)

$\mathrm{CH}$ de Matane

Saguenay-Lac-Saint-Jean

Complexe hospitalier de la Sagamie (Chicoutimi)

Centre Le Jeannois (Alma)

Centre Maria-Chapdelaine (Dolbeau-Mistassini)

Carrefour de santé de Jonquière

Hôtel-Dieu de Roberval

Québec

Centre hospitalier universitaire de Québec (CHUQ)

Centre hospitalier affilié universitaire de Québec

Hôpital Laval (Sainte-Foy)

CH Robert-Giffard (Beauport)

CH Saint-Joseph de la Malbaie

$\mathrm{CH}$ de Charlevoix (Baie Saint-Paul)

Mauricie et Centre-du-Québec

Centre hospitalier du Centre de la Mauricie (Shawinigan-Sud)

Centre hospitalier régional de Trois-Rivières

Hôpital Sainte-Croix (Drummondville)

Hôtel-Dieu d'Arthabaska (Victoriaville)

CSSS de la Saint-Maurice (La Tuque)

Estrie

Centre hospitalier universitaire de Sherbrooke

Centre de santé du Granit

Institut universitaire de gériatrie de Sherbrooke 
Montréal

Centre hospitalier St. Mary

Centre hospitalier Lasalle

Centre hospitalier Lachine

The Lakeshore General Hospital

Centre hospitalier de Verdun

Hôpital Catherine Booth

Centre de soins prolongés Grace Dart

Institut universitaire de gériatrie de Montréal

Centre hospitalier universitaire de Montréal

Centre hospitalier Fleury

CHU Mère-Enfant Sainte-Justine

Hôpital du Sacré-Cœur de Montréal

Institut de cardiologie de Montréal

Institut Philippe-Pinel de Montréal

Hôpital Louis-H.-Lafontaine

Hôpital Rivère-des-Prairies

Hôpital de Montréal pour enfants du Centre universitaire de santé McGill

Hôpital général Juif

Hôpital de réadaptation Lindsay

Centre hospitalier Richardson

Hôpital Santa Cabrini

Outaouais

Centre hospitalier des Vallées-de-l'Outaouais (Hôpital de Hull)

Centre de santé Vallée-de-la-Gatineau (Maniwaki)

CH-CHSLD de Papineau (Buckingham)

Centre de santé Pontiac (Mansfield)

Abitibi-Témiscamingue

Centre hospitalier Vallée-de-l'Or et de soins psychiatriques (Val-d'Or)

Centre hospitalier Hôtel-Dieu d'Amos

Centre hospitalier Rouyn-Noranda

Réseau de la santé et des services sociaux des Aurores boréales (La Sarre)

Centre de santé Sainte-Famille (Ville-Marie)

\section{Côte-Nord}

Centre hospitalier régional de Sept-îles

Centre hospitalier régional de Baie-Comeau

Centre de santé de la Basse-Côte-Nord (Blanc-Sablon) 
Gaspésie-Îles-de-la-Madeleine

Centre de santé de la Haute-Gaspésie (Sainte-Anne-des-Monts)

Centre hospitalier Baie-des-Chaleurs (Maria)

Centre hospitalier de Chandler

Centre hospitalier de l'Archipel - CLSC des Îles (Cap-aux-Meules)

Centre hospitalier de Gaspé

Chaudière-Appalaches

Centre hospitalier Beauce-Etchemin (Saint-Georges)

Centre de santé Paul-Gilbert (Charny)

Hôtel-Dieu de Montmagny

Centre hospitalier de la région de l'Amiante (Thetford Mines)

Laval

Cité de la santé de Laval

Centre hospitalier ambulatoire régional de Laval (CHARL)

Hôpital juif de réadaptation

Lanaudière

Centre hospitalier régional de Lanaudière (Joliette)

Centre hospitalier Pierre Le Gardeur (Repentigny)

Laurentides

CH-CR Antoine-Labelle - Centre Mont-Laurier (L'Annonciation)

Hôtel-Dieu de Saint-Jérôme

Établissement de santé d'Argenteuil (Lachute)

CH-CLSC-CHSLD des Sommets (Sainte-Agathe)

Centre hospitalier Saint-Eustache

Montérégie

Hôpital Charles-Lemoyne (Greenfield Park)

Centre hospitalier Pierre-Boucher (Longueuil)

$\mathrm{CH}$ régional du Suroît (Valleyfield)

Centre hospitalier Anna-Laberge (Châteauguay)

Hôpital du Haut-Richelieu (Saint-Jean-sur-Richelieu)

Hôtel-Dieu de Sorel

Réseau Santé Richelieu-Yamaska (Saint-Hyacinthe)

Hôpital Brome-Missisquoi-Perkins (Cowansville)

Centre hospitalier de Granby

Kateri Memorial Hospital Center (Kahnawake)

Terres-Cries-de-la-Baie-James

Conseil cri de la santé et des services sociaux de la Baie James 
Plusieurs personnes, provenant de divers secteurs, ont généreusement accepté de participer à la réflexion et de faire bénéficier le Groupe de travail de leurs connaissances ou de leur expérience dans l'un ou l'autre des domaines abordés dans ce rapport.

\section{CENTRES HOSPITALIERS}

Benoît Thibault, Hôpital Pierre-Le Gardeur, Lachenaie

Johanne Roch, Hôtel-Dieu de Saint-Jérôme

Yvon Gagné, RSSS des Aurores boréales, La Sarre

Jean-Yves Bouchard, CH des Vallées de l’Outaouais, Gatineau

Daniel Lavoie, Hôpital Sainte-Croix, Drummondville

Gilles Loiselle, Hôpital du Sacré-Cœur de Montréal

Sylvie Boudreault, Hôpital Notre-Dame, Montréal

Galina Benzeguir, Hôpital Notre-Dame, Montréal

Pierre Quintal, CH Pierre-Boucher, Longueuil

Pierre Giroux, CH Pierre-Boucher, Longueuil

Pierre Tessier, Hôpital Charles-Lemoyne, Greenfield Park

Carole Leroux, Centre de santé et de services sociaux de la Haute-Yamaska

Mario Charbonneau, Hôpital Général de Montréal

Hernani Farias, H. F. Medi-Net, Montréal

Yvon Monette, consultant, Cité de la santé de Laval

Denis Blais, Hôpital Sainte-Justine, Montréal

Dorothy Moore, M.D., Hôpital de Montréal pour enfants

Caroline Quach, M.D., Hôpital de Montréal pour enfants

Johanne LeBel, Centre du diabète de la Cité de la santé de Laval

Patrick Levasseur, Centre du diabète de la Cité de la santé de Laval

Josée Poirier, Clinique de la sclérose en plaques, CHUM, Montréal

Lyne Boudreau, Association des hôpitaux du Québec

AGENCES DE DÉVELOPPEMENT DE RÉSEAUX LOCAUX DE SANTÉ ET DE SERVICES SOCIAUX

Daniel Gagné, direction de santé publique de l’Abitibi-Témiscamingue

Louise Durand, direction de santé publique de Montréal

Roger Jacob, service des immobilisations, Montréal

Pierre Chénier, service de la gestion des actifs, Montréal

François Clossey, services administratifs, Laval

Nicole Damestoy, M.D., direction de santé publique de Laval

Guy Banville, ressources matérielles réseau, Montérégie 
ASSOCIATION DES CLSC ET DES CHSLD DU QUÉBEC

Denis Saint-Amand

CLINIQUES MÉDICALES PRIVÉES

Lucie Chevalier, Centre de santé du Carré-Saint-Louis, Montréal

Danielle Goyette, Clinique médicale 4, Taschereau, Gatineau

Louise Gendron, Polyclinique du Cap, Cap-de-la-Madeleine

Sylvie Gauthier, clinique de médecine familiale, Polyclinique de Trois-Rivières

Mélanie Morin, Clinique médicale des Cantons, Sherbrooke

Nancy Vachon, Clinique familiale Saint-Vincent, Sherbrooke

DENTISTES

Jacques Laforce, Ordre des dentistes du Québec

Daniel Pelland, Association des chirurgiens dentistes du Québec

Quatorze dentistes de différentes régions qui ont répondu à un questionnaire anonyme

PHARMACIES

T.-Neige Nguyen, Clinique Santé, Montréal

Marcel Fortier, Pharmaprix, Montréal

Stéphane Fiset, Uniprix, Montréal

Stéphane Sansregret, Uniprix, Laval

Caroline Scardera, Uniprix, Laval

Nathalie Gauthier, Jean Coutu, La Sarre

France Vallée, Clinique Santé, Cap-de-la-Madeleine

Martine Grondin, Obonsoins, Sherbrooke

Jacques Nadeau, chaîne Pharmaprix

Nathalie Larouche, bannière Santé Services / Obonsoins

Charles Milliard, bannière Uniprix

Yvan Lagacé, division pharmacie, chaîne Wal-Mart Canada

André Dubois, bannière Groupe Essaim

Richard Mayrand, chaîne Le Groupe Jean Coutu (PJC) Inc.

Pierre Ducharme, Ordre des pharmaciens du Québec

Francine Terriault-Ladouceur, Ordre des pharmaciens du Québec

Normand Cadieux, Association québécoise des pharmaciens propriétaires

Martin Amyot, Association québécoise des pharmaciens propriétaires 
SOINS INFIRMIERS PRIVÉS À DOMICILE

Dominique Lemelin, Servir + , Longueuil

Jocelyn Héon, Service + , Longueuil

ACUPUNCTEURS

Alain Migneault, Ordre des acupuncteurs du Québec

Daniel Letendre, Ordre des acupuncteurs du Québec

THANATOLOGUES

Nathalie Samson, Corporation des thanatologues du Québec

Six thanatologues de diverses régions qui ont répondu à un questionnaire anonyme

VÉTÉRINAIRES

Sylvie Latour, Ordre des médecins vétérinaires du Québec

ÉLECTROLYSTES

Deux électrolystes de Montréal

Corinne Bonfond, Association des électrolystes du Québec

Caroline Rosa, Association des électrolystes du Québec

TATOUEURS ET PERCEURS

Manon Giasson, Puce Rock, Trois-Rivières

Nathalie Duquette, Nephtys Tatoo, Sherbrooke

Martin Lamarche, Nephtys Tatoo, Sherbrooke

Élise Larocque-Goyer, Laval Tattoo Art, Laval

CENTRES D'ACCÈS AUX SERINGUES

Sylvie Ménard, Cactus-Montréal

Roxanne Beauchemin, Cactus-Montréal

Danny Raymond, Spectre de rue, Montréal

Mario Gagnon, Point de repères, Québec

Paolo Scrosati, Point de repères, Québec

François Couture, Bureau régional d'action sida, Gatineau

Lina Castonguay, Bureau régional d'action sida, Gatineau

Danielle Dupuy, Coalition sherbrookoise pour le travail de rue, Sherbrooke

Martine Dumont, CLSC de Sherbrooke

Thierry Pinet, CLSC de Sherbrooke

Johanne Ferland, $\mathrm{CH}$ régional de Trois-Rivières 
INFO-SANTÉ CLSC

Lise Brassard

HÉMA QUÉBEC

Jean-François Deschênes

CORPORATION D'URGENCES-SANTÉ

Annie Bélanger

ASSOCIATIONS DE MALADES

Louise Tremblay, Diabète Québec

Magali Plante, Société de la sclérose en plaques

COMMISSION DE LA SANTÉ ET DE LA SÉCURITÉ DU TRAVAIL (CSST)

Gilles Ricard

Julie Provencher

SERVICES DE GARDE À L'ENFANCE

Josée Poitras, CPE Complexe Desjardins, Montréal

Luc Grégoire, La Sourithèque, Montréal

Normand Richardson, CPE Idée fixe Inc., Montréal

MiLIEUX DE L'ENSEIGNEMENT

Jean-Martin Venne, UQAM

Christiane Lapointe, UQAM

Jean-Guy Tremblay, Cégep Vieux-Montréal

Daniel Martin, Commission scolaire de Montréal

MUNICIPALITÉS

Fernand Gendron, Ville de Trois-Rivières

Claude Collin, Ville de Sherbrooke

Rock Boudreault, Ville de Gatineau

J. M. Antonio Miguel, Ville de Gatineau

Michel Gagné, arrondissement Ville-Marie, Ville de Montréal

Carole Williamson, arrondissement Ville-Marie, Ville de Montréal

Pierre Dinel, arrondissement Ville-Marie, Ville de Montréal

Marc Croteau, Union des municipalités du Québec 


\section{POLICIERS}

Yvon Michaud, Association des directeurs de police du Québec

Une trentaine de directeurs de police (services de police municipaux et Sûreté du Québec) ont participé à une discussion sur le sujet dont l'Association nous a communiqué les conclusions.

\section{POSTES CANADA}

Michelle Deveau, hygiéniste industrielle

HYDRO-QUÉBEC

Christiane Renaud

Danièle Duval, Trois-Rivières

FOURNISSEURS

Clermont Goyette, Térumo (fabricant de seringues et d'aiguilles)

Réal Laberge, Systèmes médicaux BD (fabricant de seringues, d'aiguilles et de collecteurs de DBM)

Ginette Lévesque, Tyco Médical (fabricant de seringues, d'aiguilles et de collecteurs de DBM)

Johanne Winner, Tyco Médical

Louise Arseneault, Dufort et Lavigne Ltée (distributeur de fournitures médicales)

Karine Lavoie, Dufort et Lavigne (distributeur de fournitures médicales)

André Bourgeois, Les Entreprises Julien (distributeur d'autoclaves)

ÉLIMINATION DE DBM

Johanne Reney, Med-Tech Environmental Ltd.

Jean-Pierre Pépin, Med-Tech Environmental Ltd.

Johanne Tessier, BFI (site d'enfouissement sanitaire de Lachenaie)

MINISTÈRE DE L'ENVIRONNEMENT

Ginette Courtois 
Annexe II

Collecteur extérieur de seringues

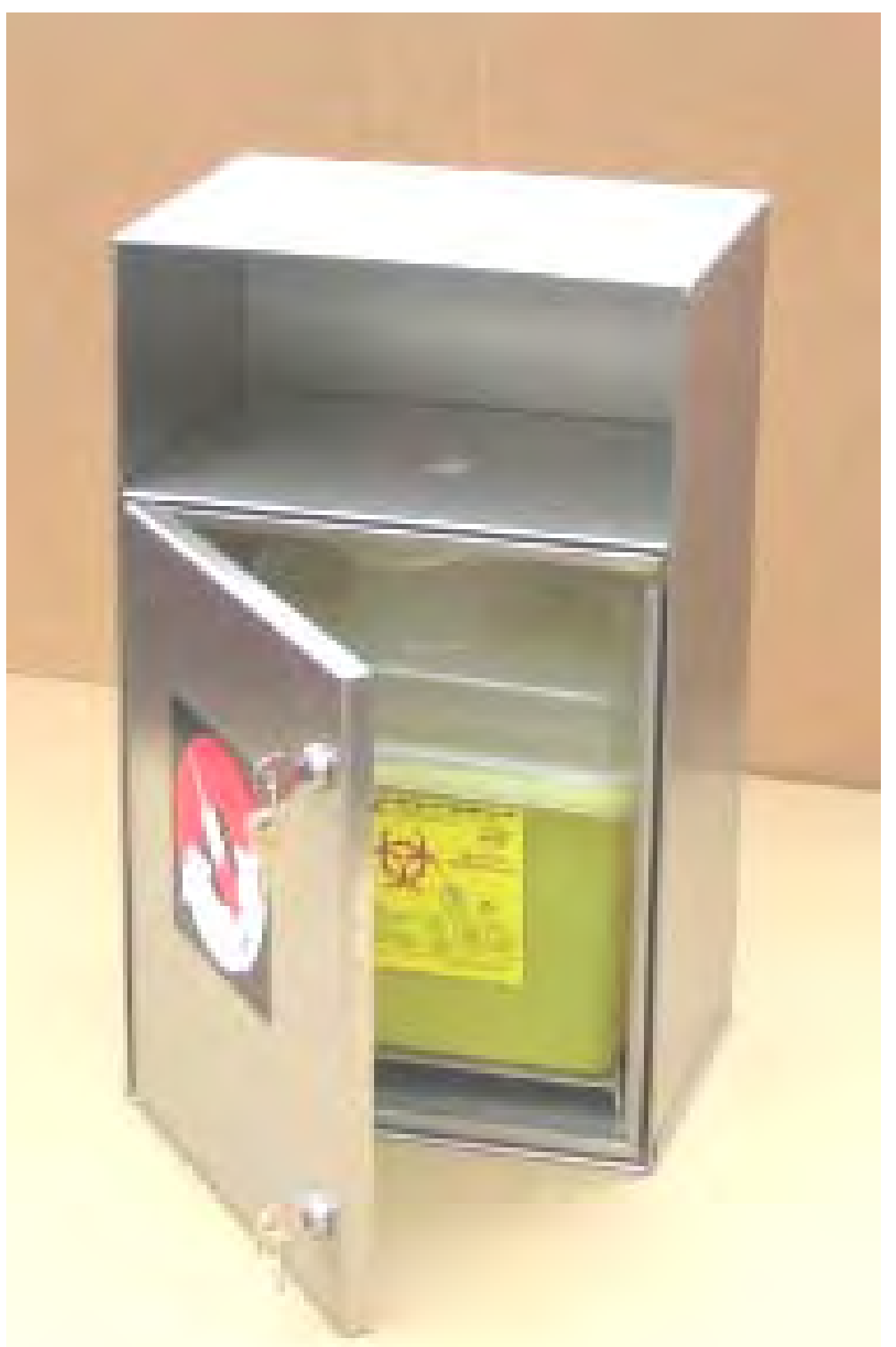



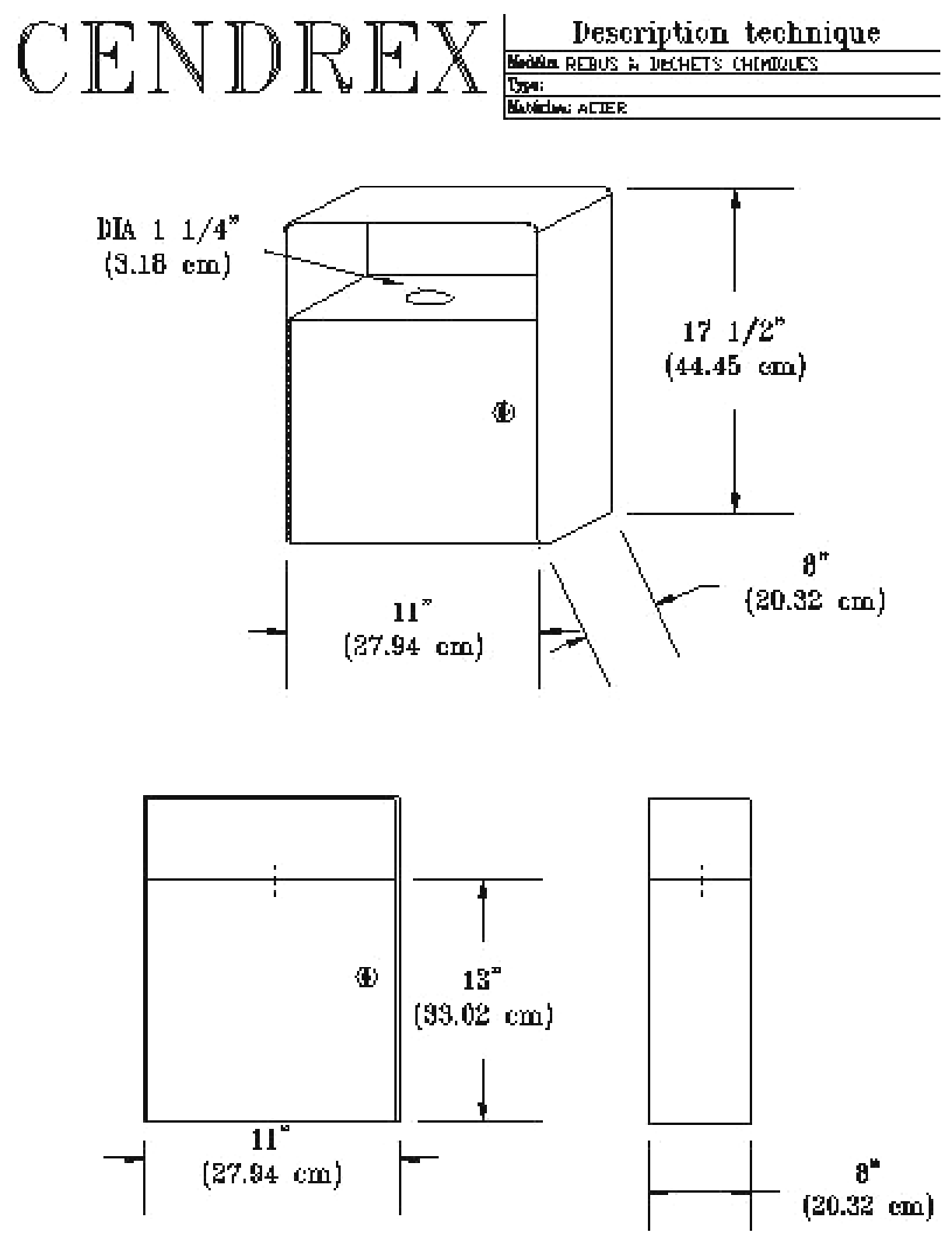
Annexe III

Autoclave

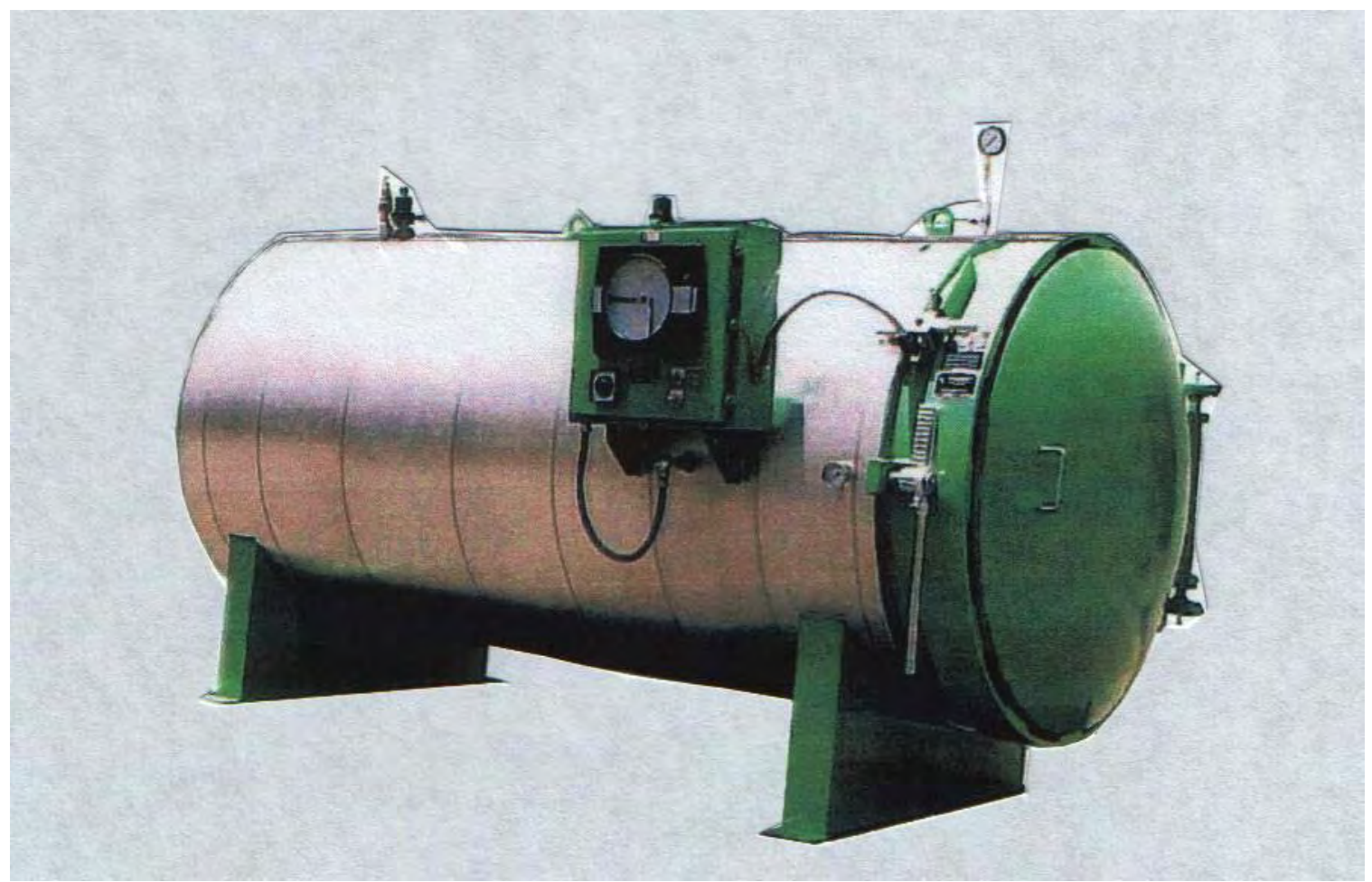





\section{Annexe IV}

\section{Modifications proposées au Règlement sur les déchets biomédicaux}

\begin{tabular}{|c|c|}
\hline Texte actuel & Modifications proposées \\
\hline $\begin{array}{l}\text { SECTION I } \\
\text { CHAMP D'APPLICATION } \\
\text { 1. Le présent règlement s'applique aux déchets } \\
\text { biomédicaux suivants: } \\
{[\ldots]} \\
3^{\circ} \text { tout déchet non anatomique constitué de l'un des } \\
\text { éléments suivants : } \\
\text { a) un objet piquant, tranchant ou cassable qui a été en } \\
\text { contact avec du sang, un liquide ou un tissu biologique, } \\
\text { provenant de soins médicaux, dentaires ou vétérinaires ou } \\
\text { d'un laboratoire de biologie médicale ou vétérinaire ou un } \\
\text { tel objet provenant de l'exercice de la thanatopraxie ; } \\
\text { [...] } \\
\text { d) un contenant de sang ou du matériel ayant été imbibé } \\
\text { de sang, provenant de soins médicaux, d'un laboratoire de } \\
\text { biologie médicale ou de l'exercice de la thanatopraxie ; }\end{array}$ & $\begin{array}{l}\text { Ajouter à cette liste l'exercice de } \\
\text { l'acupuncture, de l'électrolyse, du } \\
\text { tatouage et du perçage ainsi que les } \\
\text { autosoins ou autres activités } \\
\text { domestiques. } \\
\text { Préciser la signification de « imbibé } \\
\text { de sang ». } \\
\text { Ajouter, s'il y a lieu, les soins } \\
\text { dentaires ainsi que les activités de } \\
\text { tatouage et de perçage. }\end{array}$ \\
\hline $\begin{array}{l}\text { 2. Le présent règlement ne s'applique pas : } \\
{[\ldots]} \\
5^{\circ} \text { aux déchets biomédicaux non anatomiques provenant } \\
\text { d'activités domestiques. }\end{array}$ & Supprimer cette exclusion. \\
\hline $\begin{array}{l}\text { 3. L'article } 14 \text { [registre quotidien], le } 2^{\mathrm{e}} \text { alinéa de l'article } \\
15 \text { [rapport annuel relatif au transport], les articles } 25 \\
\text { [transporteur autorisé], } 37 \text { à } 39 \text { [véhicule à usage exclusif, } \\
\text { affiche sur le véhicule, réfrigération, cuvette de rétention, } \\
\text { compartiments en matière non poreuse], } 45 \text { à } 51 \\
\text { [désinfection après le déchargement, certificat } \\
\text { d'autorisation], } 63 \text { [abrogé] et } 64 \text { [avis de changement] ne } \\
\text { s'appliquent pas à l'exploitant d'un système de transport } \\
\text { de déchets biomédicaux, s'il en transporte moins de } 50 \mathrm{~kg} \\
\text { par mois. }\end{array}$ & $\begin{array}{l}\text { Augmenter le nombre maximum de } \\
\text { kilogrammes de déchets } \\
\text { biomédicaux piquants et tranchants } \\
\text { pouvant être transportés sans les } \\
\text { contraintes inscrites aux articles } \\
\text { mentionnés à l'article } 3 \text {. }\end{array}$ \\
\hline $\begin{array}{l}\text { Toutefois, cet exploitant n'est pas tenu de maintenir les } \\
\text { déchets biomédicaux à une température inférieure à } 4{ }^{\circ} \mathrm{C} \text {. }\end{array}$ & $\begin{array}{l}\text { Supprimer toute obligation de } \\
\text { réfrigération des déchets } \\
\text { biomédicaux piquants et tranchants. }\end{array}$ \\
\hline
\end{tabular}




\begin{tabular}{|c|c|}
\hline Texte actuel & Modifications proposées \\
\hline $\begin{array}{l}\text { SECTION II } \\
\text { GESTION DES DÉCHETS BIOMÉDICAUX } \\
\text { 11. Nul ne peut rejeter dans un réseau d'égout des } \\
\text { déchets biomédicaux. }\end{array}$ & $\begin{array}{l}\text { Ajouter l'interdiction d'en jeter dans } \\
\text { les ordures ménagères et les bacs de } \\
\text { recyclage. }\end{array}$ \\
\hline $\begin{array}{l}\text { 12. L'exploitant d'un lieu de production des déchets } \\
\text { biomédicaux doit tenir à jour un registre dans lequel il } \\
\text { inscrit chaque semaine la nature et la quantité des déchets } \\
\text { biomédicaux produits. }\end{array}$ & $\begin{array}{l}\text { À l'endroit approprié, préciser que la } \\
\text { personne physique ou morale } \\
\text { responsable d'un établissement où se } \\
\text { fait la récupération de seringues ou } \\
\text { d'aiguilles provenant d'activités } \\
\text { domestiques est considérée comme } \\
\text { l'exploitant d'un lieu de production } \\
\text { de déchets biomédicaux. } \\
\text { Pour les petits producteurs de } \\
\text { déchets biomédicaux et pour ceux } \\
\text { qui font la récupération auprès des } \\
\text { utilisateurs de seringues et } \\
\text { d'aiguilles à des fins domestiques, } \\
\text { remplacer cette disposition par celle } \\
\text { qui est contenue dans les Lignes } \\
\text { directrices sur la récupération des } \\
\text { seringues usagées : registre de } \\
\text { production tenu au rythme de } \\
\text { remplissage des contenants plutôt } \\
\text { que de façon hebdomadaire avec } \\
\text { possibilité de remplacer ce registre } \\
\text { par la facturation produite par le } \\
\text { transporteur ou le destinataire. }\end{array}$ \\
\hline $\begin{array}{l}\text { 22. Les déchets biomédicaux destinés à être expédiés } \\
\text { hors du lieu de leur production doivent être déposés dans } \\
\text { des contenants rigides, scellés et étanches. Ces contenants } \\
\text { doivent de plus être résistants à la perforation s'ils } \\
\text { contiennent des déchets biomédicaux visés au sous- } \\
\text { paragraphe } a \text { du paragraphe } 3 \text { de l'article } 1 \text {. } \\
\text { Ils doivent être maintenus dans un lieu réfrigéré à une } \\
\text { température inférieure à } 4{ }^{\circ} \mathrm{C} \text {. }\end{array}$ & $\begin{array}{l}\left.\text { Supprimer cette exigence ( } 2^{\mathrm{e}} \text { alinéa }\right) \\
\text { pour les déchets biomédicaux } \\
\text { piquants et tranchants. }\end{array}$ \\
\hline
\end{tabular}




\begin{tabular}{|c|c|}
\hline Texte actuel & Modifications proposées \\
\hline $\begin{array}{l}\text { 33. [Au lieu d'entreposage ou de traitement] Les déchets } \\
\text { biomédicaux doivent être maintenus à une température } \\
\text { inférieure à } 4{ }^{\circ} \mathrm{C} \text {. }\end{array}$ & $\begin{array}{l}\text { Supprimer cette exigence pour les } \\
\text { déchets biomédicaux piquants et } \\
\text { tranchants. }\end{array}$ \\
\hline $\begin{array}{l}\text { 39. Cet exploitant [d'un système de transport de déchets } \\
\text { biomédicaux] doit munir le véhicule utilisé: }\end{array}$ & \\
\hline $\begin{array}{l}1^{\circ} \text { d'un système de réfrigération permettant de } \\
\text { maintenir en tout temps, à une température inférieure à } \\
4^{\circ} \mathrm{C} \text {, les déchets biomédicaux qui y sont contenus ; }\end{array}$ & $\begin{array}{l}\text { Préciser que cette obligation ne } \\
\text { s'applique pas aux déchets } \\
\text { biomédicaux piquants et tranchants. }\end{array}$ \\
\hline $\begin{array}{l}\text { 40. Cet exploitant doit refuser de transporter des déchets } \\
\text { biomédicaux lorsque les obligations prévues aux articles } \\
10,22,23 \text { et } 33 \text { ne sont pas respectées. }\end{array}$ & $\begin{array}{l}\text { Faire la concordance avec la } \\
\text { modification à l'article } 33 \text {. }\end{array}$ \\
\hline
\end{tabular}





\section{Annexe V}

\section{Liste des recommandations}

1. Que le champ d'application du Règlement sur les déchets biomédicaux soit étendu à tous les producteurs de déchets biomédicaux provenant tant de l'usage domestique que des activités professionnelles ${ }^{60}$.

2. Que soit supprimée du Règlement sur les déchets biomédicaux la limite de moins de $50 \mathrm{~kg}$ de DBM par mois pouvant être transportés sans satisfaire à toutes les exigences (permis, camion réservé exclusivement à cette fin, réfrigération, système de désinfection, etc.) de telle sorte qu'elle ne puisse constituer un obstacle au transport de tous les DBM piquants et tranchants récupérés auprès des individus.

3. Que soit supprimée du Règlement sur les déchets biomédicaux toute obligation de réfrigérer les DBM piquants et tranchants.

4. Que le Règlement sur les déchets biomédicaux interdise formellement de jeter des seringues et des aiguilles usagées dans un réseau d'égout, dans les ordures ménagères et dans les bacs de recyclage.

5. Que les consignes soient les mêmes pour tous les usagers de seringues et d'aiguilles à des fins domestiques: les seringues et les aiguilles usagées doivent obligatoirement être déposées dans un contenant pour DBM piquants et tranchants et apportées à la pharmacie, au CLSC, à une clinique privée spécialisée dans le traitement de maladies qui requièrent des autosoins au moyen de seringues ou d'aiguilles ou à un organisme communautaire engagé dans la récupération de seringues, ou encore déposées dans un collecteur extérieur de seringues.

6. Que l'autorisation accordée aux dentistes de jeter leurs DBM non anatomiques aux ordures ménagères, après les avoir décontaminés sur place au moyen de leur stérilisateur pour instruments réutilisables, soit révoquée.

7. Que tous les utilisateurs d'instruments piquants et tranchants, tant à des fins professionnelles qu'à des fins domestiques, soient tenus de les déposer dans des contenants pour DBM spécifiquement prévus à cette fin.

8. Que le MSSS ou l'un de ses mandataires négocie avec les fabricants l'achat d'un nombre limité de modèles et de formats de contenants pour DBM piquants et tranchants répondant aux besoins des utilisateurs de seringues et d'aiguilles pour les autosoins.

9. Que ces contenants soient distribués gratuitement par les pharmaciens et/ou les CLSC.

60. On trouvera à l'annexe IV la liste des modifications proposées au Règlement sur les déchets biomédicaux. 
10. Que soient désignés comme lieux où les utilisateurs de seringues et d'aiguilles à des fins domestiques peuvent s'en débarrasser gratuitement et de façon sécuritaire

- les CLSC (ou autre appellation de cette mission dans les centres de santé et de services sociaux),

- les cliniques spécialisées dans le traitement de maladies qui requièrent des autosoins au moyen de seringues ou d'aiguilles,

- les pharmacies communautaires,

- les organismes communautaires engagés dans l'échange de seringues,

- les collecteurs de seringues installés dans des lieux publics.

11. Que tous ces établissements, services ou organismes, de même que les cliniques médicales privées soient tenus de récupérer et d'éliminer sans frais avec leurs propres DBM les seringues trouvées dans l'environnement apportées occasionnellement par des individus.

12. Que les CLSC soient aussi tenus de recevoir et d'éliminer sans frais avec leurs propres DBM les instruments piquants et tranchants provenant des activités de tatouage, de perçage et d'électrolyse, de même que les seringues récupérées par les organismes communautaires tels que refuges pour itinérants, maisons de jeunes et groupes de soutien en toxicomanie et celles qui sont trouvées en petite quantité dans l'environnement par des services publics (CPE, écoles, municipalité, police ou autres).

13. Que les municipalités, les établissements publics de santé, les établissements d'enseignement, les centres sportifs, les maisons de jeunes et les autres services publics ou communautaires situés dans des secteurs où l'on trouve fréquemment des seringues dans l'environnement soient invités à examiner, en collaboration avec les ressources en santé publique au besoin, la pertinence d'installer sur leur terrain un ou des collecteurs de seringues usagées. (N. B. Chaque organisme ou établissement sera responsable de fournir, installer et entretenir ces équipements.)

14. Que les propriétaires ou gestionnaires de lieux privés accessibles au public (p. ex. hôtels, restaurants, bars) soient invités à installer aux endroits appropriés des collecteurs de seringues à l'intention de leur clientèle.

15. Que tous les hôpitaux équipés d'autoclaves acceptent de décontaminer et d'éliminer sans frais les seringues et les aiguilles usagées récupérées auprès des individus par les CLSC, les cliniques spécialisées dans le traitement de maladies qui requièrent des autosoins au moyen de seringues ou d'aiguilles, les pharmacies et les organismes communautaires, de même que celles qui sont récupérées dans l'environnement par les services municipaux ou par d'autres services publics.

16. Que les hôpitaux équipés d'autoclaves soient invités à offrir à prix modique les services de décontamination et d'élimination des DBM non anatomiques provenant d'autres services professionnels (cliniques médicales, dentistes, thanatologues, acupuncteurs, centres d'hébergement privés, etc.), ce qui permettrait de compenser, du moins en partie, les frais encourus par le traitement sans frais des seringues et des aiguilles récupérées auprès des individus. 
17. Que les hôpitaux qui ne sont pas munis d'autoclave évaluent, avec l'agence de développement de réseaux locaux de services de santé et de services sociaux, la possibilité de se doter de cet appareil. (N. B. Dans les régions où les réseaux locaux couvrent de petits territoires, des ententes pourraient exister afin qu'un même hôpital muni d'un autoclave décontamine et élimine les seringues et les aiguilles récupérées auprès des individus sur des territoires de réseaux locaux voisins.)

18. Que, dès maintenant, les hôpitaux qui ne possèdent pas d'autoclave soient tenus d'éliminer sans frais avec leurs propres DBM les seringues et les aiguilles récupérées auprès des individus par ceux qui ne bénéficient pas des tarifs négociés par les services d'approvisionnement en commun (soit les pharmacies, certains organismes communautaires et, éventuellement, certaines cliniques spécialisées).

19. Que les hôpitaux s'assurent que leur personnel préposé à la manutention et au traitement des DBM reçoive une formation adéquate et qu'il bénéficie d'un environnement et de conditions de travail sécuritaires.

20. Que le ministère de la Santé et des Services sociaux effectue les démarches nécessaires auprès de la CSST afin que les équipes en santé et sécurité du travail soient mandatées pour donner de la formation aux travailleurs et aux professionnels à risque de subir des piqûres accidentelles et pour mettre en place des mesures de prévention appropriées.

21. Que les agences de développement de réseaux locaux de services de santé et de services sociaux et les CLSC, en collaboration avec les employeurs concernés, contribuent à la formation des travailleurs et des professionnels à risque et à la mise en place des mesures de prévention appropriées.

22. Que le ministère de la Santé et des Services sociaux coordonne, en collaboration avec le ministère de l'Environnement, une campagne d'information sur la récupération sécuritaire des seringues et des aiguilles usagées.

23. Que les instances régionales et locales en santé, les directions régionales du ministère de l'Environnement ainsi que les autres acteurs concernés, dont les municipalités, les associations représentant les professionnels de la santé et les associations de malades, soient associés à cette campagne d'information.

24. Que le ministère de la Santé et des Services sociaux prenne l'initiative de réunir des représentants des corps policiers, des municipalités, de la santé publique et des organismes communautaires afin de trouver les moyens les plus adéquats de concilier les actions de protection de la santé publique en matière de récupération des seringues usagées et celles qui visent la protection de la sécurité publique.

25. Que le ministère de la Santé et des Services sociaux élabore une politique nationale de récupération des seringues et des aiguilles usagées, qu'il exerce un leadership afin de mobiliser les partenaires concernés, qu'il propose des mesures concrètes, des moyens pratiques (qui doit faire quoi, comment) et qu'il alloue les ressources nécessaires pour la mise en place du système de récupération et d'élimination sécuritaires des seringues et des aiguilles usagées. 
26. Que, dans le cadre d'une politique nationale québécoise, les agences de développement de réseaux locaux de services de santé et de services sociaux prennent l'initiative de réunir les acteurs concernés pour mettre en place un système intégré pour la récupération de toutes les seringues et de toutes les aiguilles usagées sur chaque territoire de réseau local de services de santé et de services sociaux de la région. 


\section{Annexe VI}

\section{Quelques autres questions soulevées au cours de nos consultations}

1. Certains établissements s'interrogent au sujet de l'élimination du sang. Même si la réglementation le permet, ils hésitent à jeter le sang non utilisé ou non utilisable dans le réseau d'égout.

2. Certains trouvent excessive l'exigence de conserver durant trois ans les registres de production de DBM.

3. D'autres trouvent inutile l'exigence de conserver les DBM dans un local verrouillé. Ils estiment qu'il suffirait que l'endroit ne soit pas accessible au public.

4. Il a été proposé d'exiger de l'entreprise spécialisée dans le traitement et l'élimination des DBM qu'elle fournisse, pour chaque collecte, un rapport indiquant la date et l'endroit où des DBM ont été éliminés de façon sécuritaire.

5. Les thanatologues souhaiteraient recevoir de l'information au sujet des maladies à déclaration obligatoire.

6. Des tatoueurs et des perceurs souhaiteraient recevoir du Ministère des normes de bonne pratique pour éviter à leurs clients des traumatismes susceptibles de laisser des séquelles permanentes.

7. Des pharmaciens se plaignent de la façon dont sont organisés les services de récupération des médicaments périmés. Ils estiment que c'est à la fois trop (problèmes de logistique, travail non rémunéré, frais d'élimination) et pas assez (on suggère une opération annuelle «ménage de la pharmacie » rémunérée qui ne se limiterait pas à une collecte de médicaments périmés, mais où le client pourrait recevoir des conseils concernant l'utilisation de l'ensemble de ses médicaments).

8. Il a été suggéré de créer à Montréal un ou plusieurs sites d'injection supervisés, ce qui serait moins « hypocrite » que la formule qui consiste à distribuer du matériel d'injection aux UDI, mais à ne leur fournir aucun endroit où s'injecter. On soutient que cela pourrait même réduire le nombre de seringues dans l'environnement. 



\section{Bibliographie}

ASSOCIATION DES HÔPITAUX DU QUÉBEC et LABORATOIRE DE SANTÉ PUBLIQUE DU QUÉBEC. Décontamination de déchets biomédicaux de laboratoire par la vapeur, Montréal, Association des hôpitaux du Québec, 1993, 19 p.

ASSOCIATION DES HÔPITAUX DU QUÉBEC et MINISTÈRE DE LA SANTÉ ET DES SERVICES SOCIAUX. Guide de gestion des déchets comportant des risques en milieu sociosanitaire, 1990, pag. variée.

ASSOCIATION DES HÔPITAUX DU QUÉBEC et MINISTÈRE DE LA SANTÉ ET DES SERVICES SOCIAUX. Guide de gestion des déchets comportant des risques en milieu hospitalier, 1989, pag. variée.

ASSOCIATION DES INTERVENANTS EN TOXICOMANIE DU QUÉBEC. FX, 2001, 28 p.

BC CENTRE FOR EXCELLENCE ON HIV/AIDS, [en ligne].

[http://www.cfenet.ubc.ca/guide/page/sectg/consg2.html] (25 octobre 2004)

BOUCHARD, Françoise, Marie GOURDEAU et Jacinthe GAGNON. «La séroconversion d'un travailleur : impacts cliniques et économiques », Objectif prévention, vol. 25, nº 2, 2002, p. 3-6.

BOUCHARD, Françoise. « Une exposition au sang pour un travailleur : impacts économiques », Objectif prévention, vol. $25, \mathrm{n}^{\mathrm{0}} 2,2002$, p. 7-9.

CENTRE RÉGIONAL DU DIABÈTE, Technique d'injection : le stylo injecteur, Laval, Agence de développement de réseaux locaux de services de santé et de services sociaux de Laval, juin $2004.6 \mathrm{p}$.

COMITÉ DE CONCERTATION POUR LA RÉDUCTION DES SERINGUES À LA TRAÎNE DANS L'ENVIRONNEMENT À MONTRÉAL. État de situation à l'hiver 2003, 3 avril 2003, $4 \mathrm{p}$.

COMITÉ DE CONCERTATION POUR LA RÉDUCTION DES SERINGUES À LA TRAÎNE DANS L'ENVIRONNEMENT À MONTRÉAL. Les seringues à la traîne dans trois quartiers de Montréal : état de la situation - septembre 2002, 32 p.

COMMISSION D'ENQUÊTE SUR LES DÉCHETS DANGEREUX. Les déchets dangereux au Québec: Une gestion environnementale, Québec, Bureau d'audiences publiques sur l'environnement, 1990, $491 \mathrm{p}$.

DIRECTION DE LA SANTÉ PUBLIQUE DE MONTRÉAL-CENTRE. Services offerts aux UDI dans les pharmacies de Montréal et participation des pharmaciens au projet Stop Sida / UDIPharmacies, 1996 juin 1998, Montréal, Régie régionale de la santé et des services sociaux de Montréal-Centre, septembre 2000, pag. variée.

DIRECTION DE LA SANTÉ PUBLIQUE DE MONTRÉAL-CENTRE, Recommandations pour l'installation et l'utilisation de la boîte de récupération de seringues usagée, juin 2002, 6 p.

ÉMOND, Valérie. Prévalence du diabète au Québec et dans ses régions : premières estimations d'après les fichiers administratifs, Institut national de santé publique du Québec, 2002, 15 p. 
FORGET, Guylaine. «Les seringues, aiguilles et lancettes utilisées... Savez-vous comment en disposer? », Arc-en-ciel, Association du diabète Laval-Laurentides M.R.C. Des Moulins inc., cité dans Plein soleil, Diabète Québec, printemps 1998, p. 13-14.

GAGNÉ, Daniel. La récupération des seringues usagées de diabétiques en AbitibiTémiscamingue, (présentation PowerPoint), Direction de santé publique Abitibi-Témiscamingue, mai 2004.

GARCIA-ALGAR, Oscar, M.D. « Hepatitis B virus infection from a needle stick », The Pediatric Infectious Disease Journal, Vol. 16(11), novembre 1977, p. 1099, [en ligne].

[http://gateway.ut.ovid.com/gw1/ovidweb.cgi].

GAUCHER, Marcel, et Benoît ThIBAUlt. La gestion des déchets biomédicaux au Québec, ministère de l'Environnement et ministère de la Santé et des Services sociaux, tournée d'information CRSSS/MSSS, automne/hiver 1990, 10 p.

GILBERT, Hélène. Résultats de l'enquête auprès des centres hospitaliers, février et mars 2004, Groupe de travail sur la récupération des seringues usagées au Québec, Montréal, ministère de la Santé et des Services sociaux, 8 avril 2004, pag. variée.

GILBERT, Hélène. Résultats de l'enquête auprès des CLSC et CHSLD, décembre 2003janvier 2004, Groupe de travail sur la récupération des seringues usagées au Québec, Montréal, ministère de la Santé et des Services sociaux, 5 mars 2004, pag. variée.

GILBERT, Hélène. Résultats de l'enquête auprès des directions de santé publique, Groupe de travail sur la récupération des seringues usagées au Québec, Montréal, ministère de la Santé et des Services sociaux, 6 janvier 2004, 35 p.

MINISTÈRE DE LA SANTÉ ET DE LA PROTECTION SOCIALE (France) [en ligne]. [http:www.sante.gouv.fr/htm/actu/36_vih17.htm] (25 octobre 2004).

MINISTÈRE DE LA SANTÉ ET DES SERVICES SOCIAUX. Stratégie québécoise de lutte contre l'infection par le VIH et le SIDA, l'infection par le VHC et les infections transmissibles sexuellement - Orientations 2003-2009, Québec, ministère de la Santé et des Services sociaux, 2004, $56 \mathrm{p}$

MINISTÈRE DE LA SANTÉ ET DES SERVICES SOCIAUX. Les services généraux offerts par les centres de santé et de services sociaux, Québec, ministère de la Santé et des Services sociaux, septembre 2004, $179 \mathrm{p}$.

MINISTÈRE DE LA SANTÉ ET DES SERVICES SOCIAUX. Programme national de santé publique 2003-2012, Québec, ministère de la Santé et des Services sociaux, 2003, 133 p.

MINISTÈRE DE LA SANTÉ ET DES SERVICES SOCIAUX. Tatoueurs et perceurs, protégez vos clients et protégez-vous contre le VIH et les hépatites B et C, Québec, ministère de la Santé et des Services sociaux, 1999, $22 \mathrm{p}$.

MINISTÈRE DE LA SANTÉ ET DES SERVICES SOCIAUX et INSTITUT NATIONAL DE SANTÉ PUBLIQUE DU QUÉBEC. Liste officielle des centres d'accès aux seringues du Québec (distribution, vente et récupération) 2003-2004 : Programme de prévention de la transmission du VIH et des hépatites chez les utilisateurs de drogues par injection, $68 \mathrm{p}$. 
MINISTÈRE DE L'EMPLOI ET DE LA SOLIDARITÉ, SECRÉTARIAT D'ÉTAT À LA SANTÉ ET À L'ACTION SOCIALE.. Organiser dans la ville un dispositif de distribution et de récupération de seringues: Une action de terrain efficace pour la prévention du sida et des hépatites chez les usagers de drogues par voie intraveineuse, Paris, juin 1999, 39 p. (Collection $\mathrm{VIH} /$ sida).

MINISTÈRE DE L'ENVIRONNEMENT. Lignes directrices sur la récupération des seringues usagées. Québec, ministère de l'Environnement, [en ligne].

[http://www.menv.gouv.qc.ca/matieres/biomedicaux/seringues.htm] (16 septembre 2003).

MORISSETTE, Carole, et Pascale LECLERC. Monitorage des centres d'accès au matériel stérile d'injection : Rapport régional : avril 2000 à mars 2002, Montréal, Direction de santé publique de Montréal-Centre, 2003, $41 \mathrm{p}$.

NOËL, Lina, Richard CLOUTIER et Marika MUNGER. Statistiques sur les services relatifs aux programmes de prévention du VIH et des hépatites B et C offerts aux utilisateurs de drogues par injection du Québec, avril 2002 à mars 2003, ministère de la santé et des Services sociaux et Institut national de santé publique du Québec, mai 2004, 26 p.

ORDRE DES ACUPUNCTEURS DU QUÉBEC. Guide de prévention des infections dans l'exercice de l'acupuncture, Montréal, Ordre des acupuncteurs du Québec, février 2000, 24 p.

POSTES CANADA. Sécurité : Seringues hypodermiques trouvées dans le courrier, Système du manuel de la Société (2002.09), n 1605.15, 9 p.

RAYMOND, Danny. Monitorage des seringues souillées au centre-ville de Montréal : Réseau de récupération de Spectre de rue, saison 2002-2003, Montréal, [en ligne].

[http://www.santepub-mtl.qc.ca/cpmi2004/presentation/E2-Raymond.pdf].

RÉGIE RÉGIONALE DE LA SANTÉ ET DES SERVICES SOCIAUX EN ABITIBITÉMISCAMINGUE. Projet visant à faciliter la récupération des seringues utilisées à domicile en Abitibi-Témiscamingue, 22 mai 1997, 11 p.

RENÉ, Marie-Claude. Portrait national de la gestion de l'accès au matériel d'injection et de la récupération des seringues, Institut national de santé publique du Québec, janvier 2001, pag. variée.

RENÉ, Marie-Claude. Gestion de l'accès au matériel d'injection et de la récupération des seringues sur les plans canadien et international, Institut national de santé publique du Québec, janvier 2001, pag. variée.

ROBILLARD, Pierre, et autres. Évaluation de l'infectiosité des seringues à la traîne dans l'environnement : rapport d'étude pilote, Montréal, septembre 1998, 34 p.

ROCH, Johanne, et autres. Traitement des déchets biomédicaux, (présentation PowerPoint), Hôtel-Dieu de Saint-Jérôme, mai 2003.

SANTÉ CANADA. Guide de prévention des infections : Pratiques de prévention des infections dans les services personnels : tatouage, perçage des oreilles, perçage corporel et électrolyse, Ottawa, RMTC, juillet 1999, vol. 25S3, 82 p. 
SQUINAZZI, F., S. CHAMARET, Y. A. COURTOIS et L. MONTAGNIER, « La présence d'anticorps anti-VIH dans des seringues usagées abandonnées sur la voie publique ou recueillies lors de programmes d'échange pour toxicomanes : Résumé », Infections transmissibles par le sang: risques professionnels et prévention, Association internationale de la sécurité sociale, 1995, p. 369-370.

VALIQUETTE, Louise, Marcel FORTIER et Carole MORISSETTE. Projet pilote de vente et de récupération de seringues en pharmacie pour les utilisateurs de drogues injectables, $1^{\text {er }}$ avril 1994-30 avril 1995, Montréal, Régie régionale de la santé et des services sociaux de MontréalCentre, 27 mai 1996, pag. variée.

VILLE DE MONTRÉAL, DIRECTION DE SANTÉ PUBLIQUE DE MONTRÉAL-CENTRE, CLSC ET ORGANISMES COMMUNAUTAIRES. Coalition pour remédier à la présence de seringues à la traîne dans le voisinage (communiqué de presse), Montréal, 30 mai 2001, 2 p. 